Publ. RIMS', Kyoto Univ.

12 (1976), 317-377

\title{
Asymptotic Behavior at Infinity for Green's Functions of First Order Systems with Characteristics of Nonuniform Multiplicity"
}

By

\author{
Mutsuhide MATSUMURA*
}

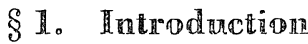

Consider a first order hyperbolic system of partial differential equations with constant coefficients

$$
\left(I \frac{\partial}{\partial t}-\sum_{j=1}^{n} A_{j} \frac{\partial}{\partial x_{j}}\right) u(\iota, x)=f(l, x), \iota \in \mathbb{R}^{1}, x \in \mathbb{R}^{n},
$$

where $I$ is the unit matrix of order $N$, the $\Lambda_{j}$ are complex $N \times N$ constant matrices, and $u(t, x), f(t, x)$ are functions whose values are $N \times 1$ (column) matrices with complex entries. The hyperbolicity means that the $N$ roots of the associated characteristic equation in $\tau$ :

$$
p(\tau, \xi) \equiv \operatorname{det}(\tau I-A(\xi))=0, A(\xi)=\sum_{j=1}^{n} \xi_{j} A_{j},
$$

are all rcal for any $\hat{\xi}$ in the real clual $\mathbb{m}^{n}$ of the real $n$-dimensional Euclidean space $\mathbb{R}^{n}$.

Our purpose is to study the asymptotic behavion at infinity of Green's function $G(x ; \lambda)$ of the stationary (or steady-state wave propagation) problem $^{2)}$ corresponding to $(1 \cdot 1)$

$$
\left(\frac{1}{i} \sum_{j=1}^{n} A_{j} \frac{\partial}{\partial x_{j}}-\lambda I\right) v(x ; \lambda)=y(x), \quad x \in \mathbb{L}^{n},
$$

where $\lambda$ is a complex parameter. The Grecn's function $G(x ; \lambda)$ for

Communicated by S. Matsuura October 20, 1975.

* Department of Mathematics, Faculty of Science, Tolyo University of F.ducation and Institute of Mathematics, The University of Tsukuba.

1) The essential part of this paper announced in Matsumura [15] and [16].

2) For more details concerning the steady-state wave propagation problem, see Schulenberger and Wilcox [19], Vainberg [23] and Wilcox [25]. 
non real $\lambda$ is defined as the inverse Fourier transform of $(A(\xi)-\lambda I)^{-1}$ :

$$
G(x ; \lambda)=(2 \pi)^{-n} \int_{g n}(A(\xi)-\lambda I)^{-1} \exp \{i\langle x, \xi\rangle\} d \xi
$$

in the sense of distributions. Here $\langle x, \xi\rangle$ denotes the inner product $x_{1} \xi_{1}+\cdots+x_{n} \xi_{n}$. We shall show the existence of outgoing and incoming Green's functions $G(x ; \sigma \pm i 0)$ of the problem $(1 \cdot 3)$ for real $\lambda=\sigma \neq 0$ and give asymptotic formulas for $|x| \rightarrow \infty$ of $G(x ; \sigma \pm i \varepsilon), \varepsilon \geq 0$ under suitable conditions on the operator $A=-i \sum_{j=1}^{n} A_{j} \partial / \partial x_{j}$. Note that $G(x ; \sigma \pm i \varepsilon)$ is a fundamental solution of the differential operator $A-(\sigma \pm i \varepsilon) I$ :

$$
(A-(\sigma \pm i \varepsilon) I) G(x ; \sigma \pm i \varepsilon)=\delta(x) 1, \quad \varepsilon \geq 0
$$

and that $G(x ; \lambda)$ is the kernel of the resolvent or Green's operator $(A-\lambda \mathbb{I})^{-1}$ in $L^{2}\left(\mathbb{R}^{n}\right)^{3)}$ when $\lambda$ is non real. Here $\delta(x)$ is the Dirac $\delta$-distribution and $\mathbb{A}$ is the closed linear operator in $L^{2}\left(\mathbb{R}^{n}\right)$ with domain $D(A)=\left\{v ; v, A v \in L^{2}\left(\mathbb{R}^{n}\right)\right\}$, defined by $A$.

The asymptotic behavior of solutions or fundamental solutions of partial differential equations with constant coefficients has been studied

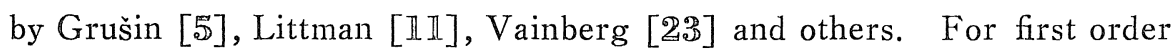
systems, it is investigated by Wilcox [25], Matsumura [1 3$]-[1$ [ 6 ] and Schulenberger and Wilcox [19].

Let $\sigma^{\circ}$ be an arbitrarily fixed non zero real number and $\delta$ a positive number such that the interval $\left[\sigma^{\circ}-\delta, \sigma^{\circ}+\delta\right]$ does not contain zero. We put $\Delta_{ \pm}=\left\{\lambda=\sigma \pm i \varepsilon ;\left|\sigma-\sigma^{\circ}\right| \leq \delta, 0 \leq \varepsilon \leq \varepsilon^{\circ}\right\}$. In the previous paper [1 14 , the author gave, under the following conditions, an asymptotic estimate for $|x| \rightarrow \infty$ of the Green's function $G(x ; \lambda)$ which is uniformly valid when $\lambda$ runs over the region $A_{ \pm}$.

(i) The $N$ roots $\lambda_{k}(\xi)$ of $p(\tau, \xi)=0$ in $\tau$ are real and have constant multiplicity for all $\xi \in \mathbb{G}^{n} \backslash\{0\}$ : i.e.,

$$
p(\tau, \xi)=\left(\tau-\lambda_{1}(\xi)\right)^{\alpha_{1}} \cdots\left(\tau-\lambda_{m}(\xi)\right)^{\alpha_{m}}, \alpha_{1}+\cdots+\alpha_{m}=N
$$

where we label the roots $\lambda_{k}(\xi)$ in decreasing order:

3) $L^{2}\left(\mathbb{R}^{n}\right)$ denotes the Hilbert space of $\mathbb{C}^{N}$-valued square integrable functions on $\mathbb{R}^{n}$ with norm

$$
\|v\|=\left\{\int_{\mathbb{R} n}|v(x)|^{2} d x\right\}^{1 / 2} .
$$




$$
\lambda_{1}(\xi)>\lambda_{2}(\xi)>\cdots>\lambda_{m}(\xi) \text { for all real } \xi \neq 0 \text {. }
$$

(ii) A root $\lambda_{k}(\xi)$ vanishes for some real $\xi \neq 0$ if and only if it vanishes identically.

(iii) The matrix $A(\xi)=\sum_{j=1}^{n} \xi_{j} A_{j}$ is semi-simple or diagonalizable for any $\xi \in \mathbb{E}^{n}$.

From i) and ii) it follows that the normal or slowness surface $S=\{s ; p(1, s)=0\}$ consists of $[m / 2]^{4)}$ disjoint sheets $S_{k}=\left\{s ; \lambda_{k}(s)=1\right\}$, $k=1, \cdots,[m / 2]$ which are $C^{\infty}$ closed hypersurfaces in $\mathbb{G}^{n}$. Then we assume

(iv) For every $k(1 \leq k \leq[m / 2])$, the Gaussian curvature $K_{k}(s)$ of $S_{k}$ at each point $s$ is different from zero.

We shall present in $\S 2$ a simple criterion due to C. H. Wilcox for a homogeneous hyperbolic polynomial $p(\tau, \xi)$ to satisfy the conditions i) and ii). The condition iii) can be removed. We shall give in $\S 8$ a corresponding result for the case when the condition iii) is not assumed.

The main purpose of this paper is to study the asymptotic behavior at infinity of the Green's functions for a class of first order systems with self-intersections of the normal surface, more precisely for the first order systems satisfying the following conditions:

$1^{\circ} p(\tau, \xi)$ is a product of strictly hyperbolic polynomials:

$$
\begin{gathered}
p(\tau, \xi)=p_{1}(\tau, \xi) \cdots p_{r}(\tau, \xi) \\
p_{l}(\tau, \xi)=\left(\tau-\lambda_{1}^{l}(\xi)\right) \cdots\left(\tau-\lambda_{m_{l}}^{l}(\xi)\right), \quad l=1, \cdots, r
\end{gathered}
$$

where we label the roots $\left\{\lambda_{k}{ }^{l}(\xi)\right\}_{1 \leq k \leq m_{l}}$ in decreasing order:

$$
\lambda_{1}^{l}(\xi)>\cdots>\lambda_{m_{l}}^{l}(\xi) \text { for } \xi \in \mathbb{G}^{n} \backslash\{0\} \text {. }
$$

Moreover the multiplicity of the roots in $\tau$ of the equation $p(\tau, \xi)=0$ is, except $\lambda=0$, at most double for every $\xi \in \mathbb{E}^{n} \backslash\{0\}$.

$2^{\circ}$ A root $\lambda_{k}^{l}(\xi)$ vanishes for some $\xi \neq 0$ if and only if it vanishes identically.

$3^{\circ}$ Let $S_{k}{ }^{l}$ denote the hypersurface $\left\{s ; \lambda_{k}{ }^{l}(s)=1\right\}$ for $1 \leq l \leq r$, $1 \leq k \leq b_{l}=\left[m_{l} / 2\right]$. Then

$$
\begin{gathered}
\operatorname{grad} \lambda_{j}{ }^{i}(s) \neq \operatorname{grad} \lambda_{l c}{ }^{l}(s) \quad \text { for any } s \in S_{j}{ }^{i} \cap S_{k}{ }^{l}, \\
1 \leq i<l \leq r, 1 \leq j \leq b_{i}, \quad 1 \leq k \leq b_{l} .
\end{gathered}
$$

4) For a non-negative real number $l,[l]$ denotes the greatest integer not exceeding $l$. 
$4^{\circ}$ For every $l$ and $k, 1 \leq l \leq r, 1 \leq k \leq b_{l}$, the Gaussian curvature $K_{k}{ }^{l}(s)$ of $S_{k}{ }^{l}$ never vanishes anywhere in $S_{k}{ }^{l}$.

To simplify statements in Theorem 8.3 which is a main purpose of this paper, we assume the following.

$5^{\circ}$ If $S_{j}{ }^{i} \cap S_{k}{ }^{l} \neq \emptyset, 1 \leq i<l \leq r, 1 \leq j \leq b_{i}, 1 \leq k \leq b_{l}$, the matrix $A(s)$ is not diagonalizable for every $s \in S_{j}{ }^{i} \cap S_{k}{ }^{l}$.

Remmolk 1. The geometric assumption that if $S_{j}{ }^{i}$ and $S_{k}{ }^{l}$ intersect, their tangent hyperplanes do not coincide at any point of $S_{j}{ }^{i} \cap S_{k}{ }^{l} \mathrm{im}$ plies the condition $3^{\circ}$.

Themank 2. When we do not assume the condition $5^{\circ}$, we must classify the possible cases.

The present work was suggested by Duff [1]. Note that there is a work [12] by Ludwig and Granoff on the propagation of singularities for first order hyperbolic systems with characteristics of nonuniform multiplicity. However, the contents and the treatment are quite different from ours. We shall indicate the plan of this paper by giving below the table of contents. Finally, the author should like to thank S. Wakabayashi for his contribution to this paper. The proof of Theorem 7. 1 on the Gaussian curvature is due to him.

\section{Contents}

$\S 1$. Introduction

§2. Hyperbolic operators and systems

§3. Spectral representations of matrices

$\$ 4$. Method of stationary phase

$\S 5$. Asymptotic behavior at infinity of the Fourier transform of a measure concentrated on a hypersurface

§6. Basic formulas I

$\S 7$. Basic formulas II

$\S 8$. Formulas for the asymptotic behavior of Green's functions at infinity

\section{§. HHyperbolic Operators and Systems}

In this section we recall some definitions and relations on the hyperbolicity in order to clarify the situation for the conditions which 
we have imposed on the system $(1 \cdot 1)$.

Given a linear differential operator $P\left(D_{t}, D_{x}\right)=\sum_{k+|a| \leq m} a_{k, a} D_{t}{ }^{k} D_{x}{ }^{\alpha}$ of order $m$ in $(n+1)$ variables $(t, x) \in \mathbb{R}^{n+1}$ with constant coefficients, the corresponding polynomial $P(\tau, \xi)$ obtained replacing $D_{t}=-i \partial / \partial t, D_{x}$ $=\left(-i \partial / \partial x_{1}, \cdots,-i \partial / \partial x_{n}\right)$ by the variables $\tau, \xi$ is called its characteristic polynomial. The principal part of $P\left(D_{t}, D_{x}\right)$ is defined as the homogeneous part of order $m$ in $\left(D_{t}, D_{x}\right)$ and denoted by $P_{n}\left(D_{t}, D_{x}\right)$.

Definition 2. 1. The opcrator $P\left(D_{t}, D_{x}\right)$ is said to be hyperbolic if $P_{m}(1,0)=a_{m, 0} \neq 0$ and $P(\tau, \xi) \neq 0$ when $\xi \in \mathbb{G}^{n}$ and $\operatorname{lm} \sigma$ is less than some fixed number. A system of differential operators $\mathbb{P}\left(D_{i}, D_{x}\right)=\left(P_{j k}\left(D_{l}\right.\right.$, $\left.D_{x}\right)_{j, k=1, \ldots, N}$ is said to bc hyperbolic if $\operatorname{det}\left(D_{t}, D_{x}\right)$ is hypcrbolic.

If $P\left(D_{t}, D_{x}\right)$ is hyperbolic, it follows that $P(\tau, \hat{\xi}) \neq 0$ when $\tilde{\xi} \in \mathbb{G}^{n}$ and $|\operatorname{Im} \tau|$ is greater than some fixed number $\geq 0$. Thus the zeros of $P(\tau, \xi)$ in $\tau$ when $\tilde{\xi}$ runs over $\mathscr{G}^{n}$ all lie in a strip $\left\{\tau ;|\operatorname{Im} \tau| \leq{ }^{\exists} s_{0}<+\infty\right\}$ of the complex $\tau$-plane. From this we can easily see that a homogeneous operator $P\left(D_{\iota}, D_{x}\right)$ is hyperbolic if and only if $P(1,0) \neq 0$ and the equation $P(\tau, \xi)=0$ in $\tau$ has only real roots when $\xi \in \mathbb{F}^{n}$ (i.e. $s_{\circ}=0$ ). If $P\left(D_{t}, D_{x}\right)$ is hyperbolic, it follows that the principal part $P_{n}\left(D_{t}, D_{x}\right)$ is also hyperbolic. But the converse is not always true. The problem of characterizing the lower order terms one may add to a homogeneous hyperbolic polynomial or matrix without loss of the hypcrbolicity is solved by Svenssons [22] for the single operators and Volevič systems with constant coefficients. On the other hand, Kasahara and Yamaguti [8] introduced the notion of strong hyperbolicity and gave an algebraic characterization of strong hyperbolicity for the Kowalevsky systems with constant coefficients. (See also Yamaguti and Kasahara [26], Strang [21] and Svensson [22].)

Definition 2.2. 1 differenital operator $P\left(D_{t}, D_{x}\right)=P_{m}\left(D_{t}, D_{x}\right)$ $+Q\left(D_{t}, D_{x}\right)$ is said to be strongly hyperbolic if $P_{m}\left(D_{\iota}, D_{x}\right)+Q^{\prime}\left(D_{\iota}, D_{x}\right)$ is hyperbolic for any choice of the lower order operator $Q^{\prime}\left(D_{t}, D_{x}\right)$. $A$ first order system $I D_{t}-\sum_{j=1}^{n} A_{j} D_{x_{j}}+B$ is called strongly hyperbolic if 
$I D_{t}-\sum_{j=1}^{n} A_{j} D_{x_{j}}+B^{\prime}$ is hyperbolic for any choice of the matrix $B^{\prime}{ }^{5)}$

A differential operator $P\left(D_{t}, D_{x}\right)$ is strongly hyperbolic if and only if the roots of the equation $P_{m}(\tau, \xi)=0$ in $\tau$ are all real and distinct for every $\xi \in \mathbb{G}^{n} \backslash\{0\}$. A differential operator $P\left(D_{t}, D_{x}\right)$ having this property is called strictly hyperbolic. A first order system $L=I D_{t}-\sum_{j=1}^{n} A_{j} D_{x_{j}}$ $+B$ is said to be strictly hyperbolic if the $\operatorname{roots}$ of $\operatorname{det}(\tau I-A(\xi))=0$ in $\tau$ are all real and distinct for every $\xi \in \mathbf{G}^{n} \backslash\{0\} .^{6)}$ Thus strong hyperbolicity is equivalent to strict hyperbolicity for a single differential operator $P\left(D_{t}, D_{x}\right)$. But this is not true for systems. A first order system $L=E D_{t}-\sum_{j=1}^{n} A_{j} D_{x_{j}}+B$ is called symmetric if $E, A_{j}, j=1, \cdots, n$ are Hermitian and if $E$ is positive definite. A first order symmetric hyperbolic system is always strongly hyperbolic but not necessarily strict. The following characterization of strongly hyperbolic first order systems is due to Yamaguti and Kasahara [26].

A first order system $L=I D_{t}-\sum_{j=1}^{n} A_{j} D_{x_{j}}+B$ is strongly hyperbolic if and only if the following conditions on the matrix $A(\xi)=\sum_{j=1}^{n} \xi_{j} A_{j}$ are satisfied.

(a) All characteristic roots of $A(\xi)$ are real for any $\xi \in \Omega=\{\xi ;|\xi|$ $=1\}$.

(b) $A(\xi)$ is uniformly diagonalizable on $\Omega$, that is, there exists a diagonalizor $N(\xi)$ of $A(\xi)$ such that $N(\xi) A(\xi) N(\xi)^{-1}$ is a diagonal matrix, the row vectors of $N(\xi)$ are of length 1 and $|\operatorname{det} N(\xi)| \geq C$, where $C$ is a positive constant independent of $\xi \in \Omega$.

Our assumptions i) and iii) on the first order system $L=I D_{t}$ $-\sum_{j=1}^{n} A_{j} D_{x_{j}}$ mean that $L$ is a strongly hyperbolic operator of constant multiplicity. Now we present a criterion due to C. H. Wilcox for a hyperbolic polynomial to be of constant multiplicity and satisfy the condition ii).

Let $a(\tau, \xi)$ be a homogeneous hyperbolic polynomial of order $N$. Then $a(\tau, \xi)$ has a factorization

$$
a(\tau, \xi)=a_{1}^{\beta_{1}}(\tau, \xi) \cdots a_{r}^{\beta_{r}}(\tau, \xi)
$$

5), 6) The notion of strong and strict hyperbolicity can be defined analogously for higher order Kowalevsky or Volevic systems. However we restrict, for simplicity, ourselves to the first order systems to be treated later on. 
where the factors $a_{j}(\tau, \xi)$ are distinct homogeneous polynomial in $(\tau, \xi)$, irreducible over the real number field. Since we can assume $a(1,0)=1$, the factors $a_{j}(\tau, \xi)$ are unique apart from their order by requiring $a_{j}(1,0)=1$. Let

$$
b(\tau, \xi)=a_{1}(\tau, \xi) \cdots a_{r}(\tau, \hat{\xi})
$$

denote the corresponding polynomial with simple irreducible factors.

Theorem 2.3. (Wilcox [24]) A necessary and sufficient condition in order that the $N$ roots of the equation $a(\tau, \xi)=0$ have conslant multiplicity for all $\xi \in \mathbb{\Xi}^{n} \backslash\{0\}$ and satisfy the condition ii) is that the normal or slowness surface $S=\left\{s=\left(s_{1}, \cdots, s_{n}\right) ; a(1, s)=0\right\}$ is bounded and $\langle s, \underset{\hat{s}}{\operatorname{grad}} b(1, s)\rangle \neq 0$ when $s \in S$.

A first order symmetric hyperbolic system whose characteristic polynomial det $(\tau I-A(\xi))$ has this property is named uniformly propagative by C. H. Wilcox. A proof of sufficiency of the theorem is given in $\S 4$ of Wilcox [24]. The necessity easily follows the following (act $\left.{ }^{7}\right)$ proved by Matsuura [17].

Let $a(\tau, \xi)$ be a homogeneous hyperbolic polynomial of constant multiplicity, that is, in the factorization of $a(\tau, \xi)$ into linear factors in $\tau: a(\tau, \xi)=\left(\tau-\lambda_{1}(\xi)\right)^{\alpha_{1}} \cdots\left(\tau-\lambda_{q}(\xi)\right)^{\alpha_{q}}{ }_{2} c_{1}+\cdots+\alpha_{q}=N$, the roots $\lambda_{j}(\xi)$ are all real and distinct for every $\xi \in \mathbb{G}^{n} \backslash\{0\}$. Then we have

$$
b(\tau, \xi)=a_{1}(\tau, \xi) \cdots a_{r}(\tau, \xi)=\left(\tau-\lambda_{1}(\xi)\right) \cdots\left(\tau-\lambda_{q}(\xi)\right) .
$$

We conclude this section with two examples of hyperbolic systems of first order.

Example 1. Consider the following hyperbolic matrix.

$$
\tau I-A(\xi)=\tau I-\left(\begin{array}{ccc}
\xi_{1} & -\xi_{2} & 0 \\
-\xi_{2} & 0 & -\xi_{2} \\
0 & 0 & 0
\end{array}\right)=\left(\begin{array}{ccc}
\tau-\xi_{1} & \xi_{2} & 0 \\
\xi_{2} & \tau & \xi_{2} \\
0 & 0 & \tau
\end{array}\right) .
$$

The characteristic polynomial is of the form.

7) See also Kitahara, Asymptotic wave functions and energy distributions for symmetric hyperbolic systems of first order (Appendix), to appear. 


$$
p(\tau, \xi)=\operatorname{det}(\tau I-A(\xi))=\tau\left(\tau^{2}-\xi_{1} \tau-\hat{\xi}_{2}^{2}\right) .
$$

The matrix $A(\xi)$ is diagonalizable for any $\xi$ with $|\xi|=1$, but not uniformly diagonalizable, so the corresponding hyperbolic system is not strongly hyperbolic (see Kasahara and Yamaguti [8] and Svensson [22]). This example is due to Petrowsky.

Example 2. Consider the following hyperbolic matrix.

$$
\begin{aligned}
\tau I-A(\xi)=\tau I- & \left(\begin{array}{cccc}
\xi_{1}+2 \xi_{2} & -2 \xi_{1} & -2 \xi_{1} & 0 \\
2 \xi_{2} & -\left(\xi_{1}+2 \xi_{2}\right) & \xi_{2} & -2 \xi_{1} \\
0 & 0 & -\left(2 \xi_{1}+\xi_{2}\right) & 2 \xi_{1} \\
0 & 0 & -2 \xi_{2} & 2 \xi_{1}+\xi_{2}
\end{array}\right) \\
& =\left(\begin{array}{cccc}
\tau-\left(\xi_{1}+2 \xi_{2}\right) & 2 \xi_{1} & 2 \xi_{1} & 0 \\
-2 \xi_{2} & \tau+\xi_{1}+2 \xi_{2} & \xi_{2} & 2 \xi_{1} \\
0 & 0 & \tau+2 \xi_{1}+\hat{\xi}_{2} & -2 \xi_{1} \\
0 & 0 & 2 \xi_{2} & \tau-\left(2 \xi_{1}+\xi_{2}\right)
\end{array}\right) .
\end{aligned}
$$

The characteristic polynomial is of the form.

$$
p(\tau, \xi)=\left(\tau^{2}-\xi_{1}^{2}-4 \xi_{2}^{2}\right)\left(\tau^{2}-4 \xi_{1}^{2}-\xi_{2}^{2}\right) .
$$

It is clear that the system satisfies the conditions $1^{\circ}, 2^{\circ}, 3^{\circ}$ and $4^{\circ}$. It also satisfies the condition $5^{\circ}$. Since we have

$$
\left|\begin{array}{ccc}
2 \xi_{1} & 2 \xi_{1} & 0 \\
\tau+\xi_{1}+2 \xi_{2} & \xi_{2} & 2 \xi_{1} \\
0 & \tau+2 \xi_{1}+\xi_{2} & -2 \xi_{1}
\end{array}\right|=-4 \xi_{1}^{3},
$$

$p(\tau, \xi)$ is the minimal polynomial of $A(\xi)$ for $\xi$ with $\xi_{1} \neq 0$. For $\xi_{1}=1 / \pm \sqrt{5}$ and $\xi_{2}=1 / \pm \sqrt{5}$, we have $p(\tau, \xi)=\left(\tau^{2}-1\right)^{2}$. Therefore the matrix $A(\xi)$ is not diagonalizable for such $\xi$.

\section{$\S 3$. Spectral Representations of Matrices}

In this section we shall make no distinction between a linear operator on the $N$-dimensional complex number space $\mathbb{C}^{N}$ and the corresponding representation matrix in the canonical base, since we shall not change the base. Let $p(\tau, \xi)$ be the characteristic polynomial $\operatorname{det}(\tau I-A(\xi))$ of a matrix $A(\xi)=\sum_{j=1}^{n} \xi_{j} A_{j}$, where the $A_{j}$ are complex $N \times N$ constant 
matrices and $\xi \in \mathbb{E}^{n}$. Suppose that the $N$ eigenvalues $\lambda_{k}(\xi)$ of $A(\xi)$ are real and have constant multiplicity for all real $\tilde{\xi} \in \mathbb{E}^{n} \backslash\{0\}$, in other words, $p(\tau, \xi)$ is a hyperbolic polynomial of constant multiplicity:

$$
p(\tau, \xi)=\left(\tau-\lambda_{1}(\xi)\right)^{\alpha_{1}} \cdots\left(\tau-\lambda_{m}(\xi)\right)^{\alpha_{m}}, \alpha_{1}+\cdots+\alpha_{m}=N,
$$

where the eigenvalues $\lambda_{k}(\xi)$ are enumerated in decreasing order:

$$
\lambda_{1}(\xi)>\lambda_{2}(\xi)>\cdots>\lambda_{m}(\xi) \quad \text { for every } \xi \in \mathbb{E}^{n} \backslash\{0\} .
$$

Since $\lambda_{k}(\xi)$ satisfies

$$
(\partial / \partial \tau)^{\alpha_{k-1}} p\left(\lambda_{k}(\xi), \xi\right)=0 \quad \text { and } \quad(\partial / \partial \tau)^{\alpha_{k}} p\left(\lambda_{k}(\xi), \xi\right) \neq 0 \quad \text { for } \quad \xi \neq 0 \text {, }
$$

it follows from the implicit function theorem that $\lambda_{k}(\xi)$ is an analytic function of $\xi$ in $\boldsymbol{\xi}^{n} \in\{0\}$. Further with the enumeration $(3 \cdot 2)$, the eigenvalues $\lambda_{k}(\xi)$ are positively homogeneous of degree one and satisfy

$$
\lambda_{k}(-\xi)=-\lambda_{m-k+1}(\xi) \quad \text { and } \quad \alpha_{k}=\alpha_{m-k \perp 1}, \quad k=1, \cdots, m .
$$

These follow from $(3 \cdot 2)$ and the relation:

$$
p(\tau, c \xi)=\prod_{k-1}^{m}\left(\tau-\lambda_{k}(c \xi)\right)^{n_{k}}=c^{N} p\left(\frac{\tau}{c}, \xi\right)=\prod_{k=1}^{m}\left(\tau-c \lambda_{k}(\xi)\right)^{\alpha_{k}}
$$

for any non zero real $c$.

Under the further assumption that $\lambda_{k}(\xi)$ vanishes for some real $\xi \neq 0$ if and only if it vanishes identically, i.e.,

$(3 \cdot 4) \quad \lambda_{k}(\xi) \equiv 0 \quad$ or $\quad \lambda_{k}(\xi) \neq 0 \quad$ for any $\xi \in \mathbb{s}^{n} \backslash\{0\}, k=1, \cdots, m$, we have the following relations. When $m=2 b$ is even,

$$
\begin{gathered}
\lambda_{1}(\xi)>\cdots>\lambda_{b}(\xi)>0>\lambda_{b+1}(\xi)=-\lambda_{b}(-\xi)>\cdots>\lambda_{m}(\xi) \\
=-\lambda_{1}(-\xi) \text { for } \xi \in \mathbb{E}^{n} \backslash\{0\} .
\end{gathered}
$$

When $m=2 b+1 \quad(b=[m / 2])$ is ockl,

$$
\begin{aligned}
\lambda_{1}(\dot{\xi}) & >\cdots>\lambda_{b}(\xi)>\lambda_{b+1}(\xi) \equiv 0>\lambda_{b+2}(\xi) \\
& =-\lambda_{b}(-\xi)>\cdots>\lambda_{m}(\xi)=-\lambda_{1}(-\xi) \text { for } \xi \in \mathbb{E}^{n} \backslash\{0\} .
\end{aligned}
$$

Now let us consider the operator $P_{k}(\xi)$ in $\mathbb{C}^{N}$ defined by

$$
\begin{aligned}
& P_{k}(\xi)=\frac{1}{2 \pi i} \int_{\Gamma_{k}(\xi)}(z I-A(\xi))^{-1} d z
\end{aligned}
$$

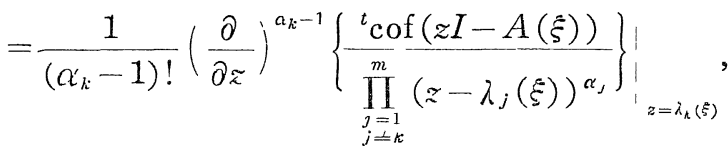


where $\Gamma_{k}(\xi)$ is a positively oriented contour enclosing the point $\lambda_{k}(\xi)$ but excluding the other points of the spectrum of $A(\xi)$, and ${ }^{t} \operatorname{cof}(z I-A(\xi))$ is the transpose of the cofactor matrix of $(z I-A(\xi))$. Then the $P_{k}(\xi)$ are analytic functions of $\xi$ in $\mathcal{G}^{n} \backslash\{0\}$ and positively homogeneous of degree zero. Moreover they have the following properties.

$$
\begin{aligned}
& P_{j}(\xi) P_{k}(\xi)=\delta_{j k} P_{k}(\xi), \\
& P_{1}(\xi)+\cdots+P_{m}(\xi)=I \text { for } \xi \neq 0, \\
& A(\xi) P_{k}(\xi)=P_{k}(\xi) A(\xi), \\
& P_{k}(-\xi)=P_{m-k+1}(\xi) .
\end{aligned}
$$

We shall denote by $M_{k}(\xi)$ the range of $P_{k}(\xi)$. Then it follows from $(3 \cdot 8)$ and $(3.9)$ that the vector space $\mathbb{C}^{N}$ can be decomposed into the direct sum of the subspaces $M_{1}(\xi), \cdots, M_{m}(\xi)$ and the operator $P_{k}(\xi)$ is the projection on $M_{k}(\xi)$ along the subspace $M_{1}(\xi) \oplus \cdots \oplus M_{k-1}(\xi)$ $\oplus M_{k+1}(\xi) \oplus \cdots \oplus M_{m}(\xi)$. The subspace $M_{k}(\xi)$ consists of all vectors $\zeta$ in $\mathbb{C}^{N}$ which satisfy the equation:

$$
\left(A(\xi)-\lambda_{k}(\xi) I\right)^{\alpha_{k}} \zeta=0,
$$

and we have

$$
\operatorname{dim} M_{k}(\xi)=\alpha_{k} .
$$

The subspace $M_{k}(\xi)$ is called the root subspace or generalized eigenspace of the operator $A(\xi)$ corresponding to the eigenvalue $\lambda_{k}(\xi)$. We now set

$$
Q_{k}(\xi)=\left(A(\xi)-\lambda_{k}(\xi) I\right) P_{k}(\xi), \quad k=1, \cdots, m .
$$

Then it is easy to see that the $Q_{k}(\xi)$ have the following properties.

$$
\begin{aligned}
& P_{j}(\xi) Q_{k}(\xi)=\hat{o}_{j k} Q_{k}(\xi) P_{j}(\xi), \\
& Q_{j}(\xi) Q_{k}(\xi)=0 \text { if } j \neq k, \\
& \left(Q_{k}(\xi)\right)^{\alpha_{k}}=0, \\
& A(\xi) Q_{k}(\xi)=Q_{k}(\xi) A(\xi) .
\end{aligned}
$$

From $(3 \cdot 9)$ and $(3 \cdot 14)$, we obtain the spectral representation of $A(\xi)$ :

$$
A(\xi)=\sum_{k=1}^{m} \lambda_{k}(\xi) P_{k}(\xi)+Q_{k}(\xi) \text {. }
$$


Let $\phi(z)$ be a holomorphic function in a domain of $\mathbb{C}$ which contains all the eigenvalues $\lambda_{k}(\xi)$ of $A(\xi)$. Then the spectral representation of the function $\phi(A(\xi))$ of $A(\xi)$ is given in the form

$$
\phi(A(\xi))=\sum_{k=1}^{m} \sum_{j=0}^{\alpha_{k}-1} \frac{\phi^{(j)}\left(\lambda_{k}(\xi)\right)}{j !}\left(Q_{k}(\xi)\right)^{j} P_{k}(\xi) .
$$

In particular we have for $\phi(z)=1 /(z-\lambda) \quad(\lambda \neq$ real $)$

$$
(A(\xi)-\lambda I)^{-1}=\sum_{k=1}^{m} \sum_{i=0}^{a_{k}-1}(-1)^{j} \frac{\left(Q_{k}(\xi)\right)^{j}}{\left(\lambda_{k}(\xi)-\lambda\right)^{j+1}} P_{k}(\xi) .
$$

If $A(\tilde{\xi})$ is diagonalizable or semi-simple for any $\xi \in \mathbb{G}^{n}$, we have

$$
\begin{gathered}
A(\xi)=\sum_{k=1}^{m} \lambda_{k}(\xi) P_{k}(\xi) \\
(A(\xi)-\lambda I)^{-1}=\sum_{k=1}^{m} \frac{1}{\lambda_{k}(\xi)-\lambda} P_{k}(\xi), \quad \lambda=\text { treal. }
\end{gathered}
$$

We shall derive a representation for the resolvent $(A(\xi)-\lambda I)^{-1}$ of the matrix $A(\xi)$ whose characteristic polynomial $p(\tau, \xi)$ satisfies the conditions $1^{\circ}, 2^{\circ}$ and $3^{\circ}$ in $\S 1$. For this purpose we first consider the case when the roots of $p(\tau, \xi)=0$ are all distinct for every $\xi \Leftarrow \xi^{n} \backslash\{0\}$. Define a function $u(\tau, \lambda, \xi)$ by

$$
\begin{aligned}
u(\tau, \lambda, \xi) & =\frac{1}{(\tau-\lambda)} \frac{1}{2 \pi i} \\
\times \int_{i(\xi)}\left(z-\lambda_{j}(\xi)\right)\left(z-\lambda_{k}(\xi)\right) & (z I-\tau)
\end{aligned}
$$

where $\gamma(\xi)$ is a positively oriented contour enclosing the points $\lambda_{j}(\xi)$ and $\lambda_{k}(\xi)$ but excluding the other points of the spectrum of $A(\xi)$. Then we have from $(3 \cdot 24)$

$$
\begin{gathered}
u(\tau, \lambda, \xi)=\frac{1}{\tau-\lambda} \frac{{ }^{t} \operatorname{cof}(\tau I-A(\xi))}{\left(\tau-\lambda_{1}(\xi)\right) \cdots_{\hat{j}} \cdots \hat{k}_{\hat{k}} \cdots\left(\tau-\lambda_{N}(\xi)\right)}, \\
u\left(\lambda_{j}(\xi), \lambda, \xi\right)=\frac{\lambda_{j}(\xi)-\lambda_{k}(\xi)}{\lambda_{j}(\xi)-\lambda} P_{j}(\xi) \\
\text { and } u\left(\lambda_{k}(\xi), \lambda, \xi\right)=\frac{\lambda_{k}(\xi)-\lambda_{j}(\xi)}{\lambda_{k}(\xi)-\lambda} P_{k}(\xi),
\end{gathered}
$$

where $\widehat{j}$ indicates that $\left(\tau-\lambda_{j}(\xi)\right)$ is omitted. Therefore 


$$
\begin{aligned}
(A(\xi)-\lambda I)^{-1}= & \frac{u\left(\lambda_{j}(\xi), \lambda, \xi\right)}{\lambda_{j}(\xi)-\lambda_{k}(\xi)}-\frac{u\left(\lambda_{k}(\xi), \lambda, \xi\right)}{\lambda_{j}(\xi)-\lambda_{k}(\xi)} \\
& +\sum_{\substack{l \neq 1 \\
l \neq j, k}}^{N} \frac{1}{\lambda_{k}(\xi)-\lambda} P_{k}(\xi) .
\end{aligned}
$$

Now, we know that

$$
\begin{aligned}
& \frac{u\left(\lambda_{j}(\xi), \lambda, \xi\right)}{\lambda_{j}(\xi)-\lambda_{k}(\xi)}-\frac{u\left(\lambda_{k}(\xi), \lambda, \xi\right)}{\lambda_{j}(\xi)-\lambda_{k}(\xi)} \\
& =\int_{0}^{1} \frac{\partial u}{\partial \tau}\left(\vartheta \lambda_{j}(\xi)+(1-\vartheta) \lambda_{k}(\xi)\right) d \vartheta .
\end{aligned}
$$

To simplify formulae, we introduce the notation:

$$
\begin{gathered}
\lambda_{j k}(\xi, \vartheta)=\vartheta \lambda_{j}(\xi)+(1-\vartheta) \lambda_{k}(\xi), \\
P_{j k}(\xi, \vartheta)^{s)}=\frac{1}{2 \pi i} \int_{1(\xi)} \frac{\left(z-\lambda_{j}(\xi)\right)\left(z-\lambda_{k}(\xi)\right)}{\left(z-\lambda_{j k}(\xi, \vartheta)\right)}(z I-A(\xi))^{-1} d z \\
=\left.\frac{{ }^{t} \operatorname{cof}(\tau I-A(\xi))}{\left(\tau-\lambda_{1}(\xi)\right) \cdots_{j} \cdots \bar{k}_{\hat{k}} \cdots\left(\tau-\lambda_{N}(\xi)\right)}\right|_{\tau=\lambda_{j k}(\xi, \vartheta)},
\end{gathered}
$$

$$
\begin{aligned}
R_{j k}(\xi, \vartheta) & =\frac{1}{2 \pi i} \int_{r(\xi)} \frac{\left(z-\lambda_{j}(\xi)\right)\left(z-\lambda_{k}(\xi)\right)}{\left(z-\lambda_{j k}(\xi, \vartheta)\right)^{2}}(z I-A(\xi))^{-1} d z \\
& =\left.\frac{\partial}{\partial \tau}\left\{\frac{{ }^{t} \operatorname{cof}(\tau I-A(\xi))}{\left(\tau-\lambda_{1}(\xi)\right) \cdots \hat{\jmath}_{\hat{j}} \cdots \hat{k}_{\hat{k}} \cdots\left(\tau-\lambda_{N}(\xi)\right)}\right\}\right|_{\tau=\lambda_{j k}(\xi, \vartheta)} .
\end{aligned}
$$

Then we obtain the representation:

$$
\begin{aligned}
(A(\xi) & -\lambda I)^{-1}=\int_{0}^{1}\left\{-\frac{1}{\left(\lambda_{j k}(\xi, \vartheta)-\lambda\right)^{2}} P_{j k}(\xi, \vartheta)\right. \\
& \left.+\frac{1}{\left(\lambda_{j k}(\xi, \vartheta)-\lambda\right)} R_{j k}(\xi, \vartheta)\right\} d \vartheta+\sum_{\substack{l \neq 1 \\
l \neq j, k}}^{N} \frac{1}{\lambda_{l}(\xi)-\lambda} P_{l}(\xi)
\end{aligned}
$$

where $P_{j k}(\xi, \vartheta)$ and $R_{j k}(\tilde{\xi}, \vartheta)$ are $C^{\infty}$ functions in ( $\left.{ }^{n} \backslash\{0\}\right) \times[0,1]$ and positively homogeneous of degree 1 and 0 in $\xi$ respectively.

We consider the case when in an open subset $U$ of $\xi^{n} \backslash\{0\}, \lambda_{j}(\xi)$ and $\lambda_{k}(\xi)$ may coincide for some $\xi$ but the other eigenvalues are all simple for any $\xi \in U$. Then the above representation $(3 \cdot 32)$ remains valid in this open set $U$. Let us now establish a general formula for 8) $P_{j k}(\xi, 0)=P_{k}(\xi), P_{j k}(\xi, 1)=P_{j}(\xi)$ 
$A(\xi)$ satisfying the conditions $1^{\circ}, 2^{\circ}$ and $3^{\circ}$ in $\S 1$. To simplify the description, we change some notations. Taking account of the relations corresponding to $(3 \cdot 5)$ and $(3 \cdot 6)$ for the roots of $p_{l}(\tau, \xi)=0$ in $\tau$, we reenumerate the roots of $p(\tau, \xi)=0$ in $\tau$ in the following way.

$$
p(\tau, \xi)=\left(\tau-\lambda_{0}(\xi)\right)^{a} \prod_{|k|=1}^{b}\left(\tau-\lambda_{k}(\xi)\right), a+2 b=N,
$$

where $\lambda_{0}(\xi) \equiv 0, \lambda_{k}(\xi)>0$ and $\lambda_{-k}(\xi)<0$ for $\stackrel{\xi}{\xi} \in \mathbb{G}^{n} \backslash\{0\}, k=1,2, \cdots, b$ and $\lambda_{k}(-\xi)=-\lambda_{-k}(\xi), k=1, \cdots, b$.

Thus the $\lambda_{k}{ }^{l}(\xi), l=1, \cdots, r, k=1, \cdots, b_{l}=\left[m_{l / 2}\right]$ are in a suitable way reelummerated as $\lambda_{1}(\xi), \cdots, \lambda_{b}(\xi), b=b_{1}+\cdots+b_{r}$. We also denote by $S_{k}$ the sheet $\left\{s ; \lambda_{k}(s)=1\right\}, 1 \leq k \leq b$ of the normal surface of $p(\tau, \xi)$.

Form the assumptions $1^{\circ}$ and $3^{\circ}$ in $\S 1$, it follows that every $S_{j} \cap S_{k}$ $(j<k)$ is an empty set or an $(n-2)$-dimensional smooth submanifold of $S_{j}\left(S_{k}\right)$. In fact, suppose that $s^{\circ} \in S_{j} \cap S_{k}$, i.e., $\lambda_{j}\left(s^{\circ}\right)=\lambda_{k}\left(s^{\circ}\right)=1$. From the condition $3^{\circ}$ we may assume without loss of generality that $\partial \lambda_{j}\left(s^{\circ}\right) / \partial \xi_{n}$ $\neq \partial \lambda_{k}\left(s^{\circ}\right) / \partial \xi_{n}$. By the implicit function theorem there exists a $C^{\infty}$ function $s_{n}=f\left(s^{\prime}\right)$ defined in a neighborhood of $s^{\circ}$ such that $\lambda_{j}\left(s^{\prime}, f\left(s^{\prime}\right)\right)$ $=\lambda_{k}\left(s^{\prime}, f\left(s^{\prime}\right)\right)$ and $s_{n}{ }^{\circ}=f\left(s^{\circ}\right)$. Consider the equation $\lambda_{j}\left(s^{\prime}, f\left(s^{\prime}\right)\right)=1$. Then

$$
\begin{gathered}
\underset{s^{\prime}}{\left.\operatorname{grad} \lambda_{j}\left(s^{\prime}, f\left(s^{\prime}\right)\right)\right|_{s^{\prime}-s^{\prime \prime}}}=\left(\frac{\partial \lambda_{j}}{\partial \xi_{1}}\left(s^{\circ}\right)-\frac{\partial \lambda_{j}}{\partial \xi_{n}}\left(s^{\circ}\right) \frac{\frac{\partial \lambda_{j}}{\partial \xi_{1}}\left(s^{\circ}\right)-\frac{\partial \lambda_{k}}{\partial \xi_{1}}\left(s^{\circ}\right)}{\frac{\partial \lambda_{j}}{\partial \xi_{n}}\left(s^{\circ}\right)-\frac{\partial \lambda_{k}}{\partial \xi_{n}}\left(s^{\circ}\right)}, \cdots,\right. \\
\left.\frac{\partial \lambda_{j}}{\partial \xi_{n}}\left(s^{\circ}\right)-\frac{\partial \lambda_{j}}{\partial \xi_{n}}\left(s^{\circ}\right) \frac{\frac{\partial \lambda_{j}}{\partial \xi_{n}}\left(s^{\circ}\right)-\frac{\partial \lambda_{k}}{\partial \xi_{n-1}}\left(s^{\circ}\right)}{\frac{\partial \lambda_{j}}{\partial \xi_{n}}\left(s^{\circ}\right)-\frac{\partial \lambda_{k}}{\partial \xi_{n}}\left(s^{\circ}\right)}\right) \neq 0 .
\end{gathered}
$$

For olherwise we have $\underset{\xi}{\operatorname{grad}} \lambda_{j}\left(s^{\circ}\right)=\underset{\xi}{\operatorname{grad}} \lambda_{k}\left(s^{\circ}\right)$. Thus $S_{j} \cap S_{k}$ is a $C^{\circ}$ submanifold of $S_{k}$ (and $S_{j}$ ).

Set

$$
\text { (1) }{ }_{j k}=\left\{s /|s| ; s \in S_{j} \cap S_{k}\right\} \subset \Omega=\{s ;|s|=1\}, \quad j<k \text {. }
$$

Take open subsets $V_{j k}$ and $V_{j k}^{\prime}$ of $\Omega$ such that $\omega_{j k} \subset \bar{V}_{j k}^{\prime} \subset V_{j k}$. Let $w_{j k}(\xi)$ be a $C^{\hookrightarrow}$ function on $\Omega$ such that $w_{j k}(\xi)=1$ in $\gamma_{j k}^{\prime}$ and supp $w_{j k} \subset V_{j k}$. We denote by the same letter the $C^{\infty}$ function extended to the space 
$\mathbb{F}^{n} \backslash\{0\}$ by homogeneity of degree $0: w_{j k}(\xi)=w_{j k}(\xi /|\xi|)$. Note that we can choose $V_{j k}$ and $V_{j k}^{\prime}$ as small as desired.

Making use of the functions $w_{j k}$ for $(j, k)$ such that $j<k$ and $S_{j} \cap S_{k} \neq \emptyset$, we obtain the following representation which is valid for $A(\xi)$ whose characteristic polynomial $p(\tau, \xi)$ satisfies the conditions $1^{\circ}$, $2^{\circ}$ and $3^{\circ}$ in $\S 1$.

$$
\begin{aligned}
& (A(\xi)-\lambda I)^{-1}=\sum_{j, k}^{*}\left[\int _ { 0 } ^ { 1 } w _ { j k } ( \xi ) \left\{-\frac{P_{j k}(\xi, \vartheta)}{\left(\lambda_{j k}(\xi, \vartheta)-\lambda\right)^{2}}\right.\right. \\
& \left.\quad+\frac{R_{j k}(\xi, \vartheta)}{\left(\lambda_{j k}(\xi, \vartheta)-\lambda\right)}\right\} d \vartheta+\left(1-w_{j k}(\xi)\right)\left\{\frac{P_{j}(\xi)}{\lambda_{j}(\xi)-\lambda}\right. \\
& \left.\left.\quad+\frac{P_{k}(\xi)}{\lambda_{k}(\xi)-\lambda}\right\}\right]+\sum_{k}^{* *} \frac{P_{k}(\xi)}{\lambda_{k}(\xi)-\lambda} .
\end{aligned}
$$

Here the sum $\Sigma^{*}$ is taken over all pairs $(j, k)$ such that $1 \leq j<k \leq b$ or $-1 \geq j>k \geq-b$, and $S_{|j|} \cap S_{|k|} \neq \emptyset$. On the other hand the sum $\Sigma^{* *}$ is taken over all $k$ such that $1 \leq|k| \leq b$ and $S_{|k|} \cap S_{j}=\emptyset$ for any $j \neq|k|$ $(1 \leq j \leq b)$. We defined $w_{-j,-k}$ by $w_{-j,-k}(\xi)=w_{j k}(-\xi), 1 \leq j, k \leq b$.

Note that if $A\left(\xi^{\circ}\right)$ is diagonalizable for some $\xi^{\circ} \in S_{j} \cap S_{k}$, we have

$$
P_{j k}\left(\xi^{\circ}, \vartheta\right)=0
$$

\section{§4. Method of Stationary Phase}

In this section we first present some basic facts concerning the method of stationary phase.

Let $G$ be a bounded domain in the real $m$-dimensional Euclidean space $\boldsymbol{s}^{m}$, and let $h$ be a real valued $C^{\infty}$ function on $G$. A point $a \in G$ is called a critical or stationary point of $h$ if $\operatorname{grad} h(a)=\left(\partial h(a) / \partial \xi_{1}\right.$, $\left.\cdots, \partial h(a) / \partial \xi_{m}\right)=0$. A critical point $a$ is called non-degenerate if and only if the Hessian Hess $h(a)=\operatorname{det}\left(\partial^{2} h(a) / \partial \xi_{j} \partial \xi_{k}\right)$ of $h$ at $a$ is different from zero. The inverse function theorem implies that a non-degenerate critical point is isolated, for Hess $h(\xi)$ is the Jacobian $D(\eta) / D(\xi)$ of the map: $\xi \mapsto \eta=\operatorname{grad} h(\xi)$. We shall denote by $H(a)$ the Hessian matrix $\left(\partial^{2} h(a) / \partial \xi_{j} \partial \xi_{k}\right)$ of $h$ at $a$ and by $\operatorname{sgn} H(a)$ its signature, i.e. the number of positive eigenvalues of $H(a)$ minus the number of negative ones. 
Now we consider the integral

$$
f(t)=\int_{G} g(\xi) \exp \{i t h(\xi)\} d \xi,
$$

where $g \in C_{\circ}{ }^{\circ}(G)$. We are interested in the asymptotic behavior at $|t| \rightarrow \infty$ of $f(t)$. If $h$ has no critical points on supp $g$, the exponential oscillates rapidly for large $|t|$ and the positive and negative swings in value will tend to cancel out in the integration. In fact we can show by integration by parts that $f(t)$ approaches zero as $|t| \rightarrow \infty$ faster than any negative power of $|t|$. Thus the principal contribution to the asymptotic behavior of $f(t)$ for large $|t|$ should arise from the immediate neighborhoods of the critical points of $h(\xi)$.

Theorem 4. 1. $1^{\circ}$ If h has no critical points on supp $g$, then we have

$$
f(t)=O\left(|t|^{-\infty}\right) \text { as }|t| \rightarrow \infty .
$$

$2^{\circ} \quad$ Let $h$ have critical points on supp $g$ and assume that these points are all non-degenerate. Since non-degenerate critical points are isolated, h has only finitely many such points $a^{(1)}, \cdots, a^{(r)}$ on supp $g$. Then we have

$$
\begin{aligned}
f(t)= & (2 \pi)^{m / 2} \sum_{l=1}^{r} \frac{\exp \left\{i t h\left(a^{(l)}\right)+(i \pi / 4) \operatorname{sgn} H\left(a^{(l)}\right) \operatorname{sgn} t\right\}}{\left|\operatorname{Hess} h\left(a^{(l)}\right)\right|^{1 / 2}}|t|^{-m / 2} \\
& \times\left\{\left.\sum_{|\alpha|<2 N} C_{l, \alpha} D_{\eta}^{\alpha}\left[g\left(\varphi^{\prime}(\eta)\right) \frac{D\left(\varphi^{(l)}\right)}{D(\eta)}\right]\right|_{\eta=0}|t|^{-|a| / 2}\right\}+R_{2 N}(t),
\end{aligned}
$$

and

$$
\left|R_{2 N}(t)\right| \leq C|t|^{-m / 2-N} \quad \text { as } \quad|t| \rightarrow \infty,
$$

where $\xi=\varphi^{l}(\eta)$ is a $C^{\infty}$ change of variables in a neighborhood of $a^{(l)}$ with $\eta$ in a neighborhood of 0 in ${ }^{m}$ such that

$$
a^{(l)}=\varphi^{l}(0),\left.\frac{D\left(\varphi^{l}\right)}{D(\eta)}\right|_{\eta=0}=1
$$

and

$$
h\left(\varphi^{l}(\eta)\right)=h\left(a^{(l)}\right)+\frac{1}{2} \sum_{j, k=1}^{m} \frac{\partial^{2} h}{\partial \xi_{j} \partial \xi_{k}}\left(a^{(l)}\right) \eta_{j} \eta_{k} .
$$


Here

$$
C_{l, \alpha}=\left.\frac{1}{\alpha !}(\partial / \partial x)^{\alpha} \exp \left\{-\frac{i \operatorname{sgn} t}{2}\left\langle H\left(a^{(l)}\right)^{-1} x, x\right\rangle\right\}\right|_{x=0}
$$

vanishes for odd $|\alpha|$, and is independent of $|t|$ and $g$.

The method of stationary phase for multiple integrals has been investigated by many authors. For the sake of completeness we give a proof of this theorem (see e.g. Fedoriuk [2], Hörmander [7, 3. 2]).

Proof. $1^{\circ}$ For the differential operator

$$
M=i^{-1} \sum_{j=1}^{m} \frac{\partial h}{\partial \xi_{j}} \frac{\partial}{\partial \xi_{j}} / \sum_{k=1}^{m}\left(\frac{\partial h}{\partial \xi_{k}}(\xi)\right)^{2},
$$

we have

$$
\exp \{i t h(\xi)\}=t^{-1} M[\exp \{i t h(\xi)\}] .
$$

Using repeatedly this relation, we find

$$
\begin{gathered}
\int_{G} g(\xi) \exp \{i t h(\xi)\} d \xi=t^{-1} \int_{G}{ }^{t} M[g(\xi)] \exp \{i t h(\xi)\} d \xi \\
=\cdots=t^{-p} \int_{G}\left({ }^{t} M\right)^{p}[g(\xi)] \exp \{i t h(\xi)\} d \xi=\cdots,
\end{gathered}
$$

where ${ }^{t} M$ is the transposed operator of $M$. This means (4.2).

$2^{\circ}$ By use of a $C^{\infty}$ partition of unity we see that it suffices to consider the case when $h$ has only one non-degenerate critical point $a$ and when the support is contained in a sufficiently small neighborhond of $a$. We can write

$$
\begin{aligned}
h(\xi)-h(a) & =\sum_{j, k=1}^{m} \alpha_{j k}(\xi)\left(\xi_{j}-a_{j}\right)\left(\xi_{k}-a_{k}\right) \\
& =\frac{1}{2}\left\langle H_{\xi}(\xi-a),(\xi-a)\right\rangle,
\end{aligned}
$$

where

$$
H_{\xi}=\left(2 \alpha_{j k}(\xi)\right), \alpha_{j k}(\xi)=\int_{0}^{1}(1-\rho) \frac{\partial^{2} h}{\partial \xi_{j} \partial \xi_{k}}(a+(\xi-a) \rho) d \rho
$$

Note that the $\alpha_{j k}(\xi)$ are $C^{\infty}$ functions and that the matrix $H_{\xi}$ is sym- 
metric, i.e. ${ }^{t} H_{\xi}=H_{\xi}$ and

$$
\alpha_{j k}^{\prime}(a)=\frac{1}{2} \frac{\partial^{2} h}{\partial \xi_{j} \partial \xi_{k}}(a), \quad H_{a}=H(a) .
$$

Here and in sequel, for a matrix $H,{ }^{t} H$ denotes its transposed matrix. If we set

$$
K_{\xi}=H_{\xi}^{-1} H_{a}=l+E_{\xi},
$$

we have

$$
K_{a}=I(=i d), \quad E_{a}=0,
$$

$$
H_{\xi} K_{\xi}={ }^{t} K_{\xi} H_{\xi} \text { and } H_{\xi} E_{\xi}={ }^{\imath} E_{\xi} H_{\xi} \text {. }
$$

Define $K_{\xi}^{1 / 2}$ for $\xi$ near $a$ by

$$
K_{\xi}^{1 / 2}=\sum_{n=0}^{\infty} c_{n} E_{\xi}{ }^{n},
$$

where the $c_{n}$ are the coefficients in the expansion $(1+\rho)^{1 / 2}=\sum_{n=0}^{n} c_{n} \rho^{n}$. Then it follows from $(4 \cdot 15)$ and $(4 \cdot 16)$ that

$$
H_{\xi} K_{\xi}^{1 / 2}={ }^{1} K_{\xi}^{1 / 2} H_{\xi} \quad \text { and } \quad K_{\xi}^{1 / 2} K_{\xi}^{1 / 2}=K_{\xi} .
$$

Substituting $\xi-a=K_{\xi}^{1 / 2} \eta$ in $(4 \cdot 10)$ and making use of $(4 \cdot 17),(4 \cdot 12)$ and $(4 \cdot 13)$, we find

$$
h(\xi)-h(a)=\frac{1}{2}\left\langle H_{\xi} K_{\xi}^{1 / 2} \eta, K_{\xi}^{1 / 2} \eta\right\rangle=\frac{1}{2}\langle H(a) \eta, \eta\rangle .
$$

On the other hand we have

$$
\left.\frac{D(\eta)}{D(\xi)}\right|_{i=a}=\operatorname{det}\left(K^{1 / 2}\right)^{-1}=1
$$

Consequently, there exists the inverse function $\xi=\varphi(\eta)$ of $\eta=\left(K_{\xi}^{1 / 2}\right)^{-1}$ $\times(\xi-a)$ which is defined in a neighborhood of 0 in $\mathbb{R}^{m}$ and $\varphi(0)=a$, $D(\varphi) /\left.D(\eta)\right|_{\eta=0}=1$. Making the change of variables $\xi=\varphi(\eta)$ in $(4 \cdot 1)$, we have

$$
f(t)=\exp \{i t h(a)\} \int_{G} g_{1}(\eta) \exp \left\{\frac{i t}{2}\langle H(a) \eta, \eta\rangle\right\} d \eta
$$

where $g_{1}(\eta)=g(\varphi(\eta)) D(\varphi) / D(\eta) \in C_{\circ}{ }^{\infty}\left({ }^{(\mathrm{g}}{ }^{n}\right)$. Let us regard the integral in $(4 \cdot 19)$ as the result of applying the temperate distribution $\exp \{i t\langle H(a) \eta$, 
$\eta) / 2\}$ to the test function $g_{1}$. Then we obtain by the Fourier's inversion formula

$$
\begin{aligned}
f(t) \exp \{ & -i t h(a)\}=\left\langle\exp \left\{\frac{i t}{2}\langle H(a) \eta, \eta\rangle\right\}, g_{1}\right\rangle \\
= & \left\langle\exp \left\{\frac{i t}{2}\langle H(a) \eta, \eta\rangle\right\}, \mathscr{F}^{-1} \mathscr{F} g_{1}\right\rangle^{9)} \\
& =\left\langle\mathscr{F}^{-1}\left[\exp \left\{\frac{i t}{2}\langle H(a) \eta, \eta\rangle\right\}\right], \widehat{g}_{1}\right\rangle,
\end{aligned}
$$

where $\widehat{g}_{1}=\mathscr{F} g_{1}$. From the well known Fresnel integral

$$
\int_{-\infty}^{+\infty} \exp \left\{ \pm i \rho^{2}\right\} d \rho=\left(\frac{\pi}{2}\right)^{1 / 2}(1 \pm i)=\pi^{1 / 2} \exp \left\{ \pm \frac{i \pi}{4}\right\}
$$

we find

$$
\int_{-\infty}^{+\infty} \exp \left\{\frac{i d}{2} \rho^{2}-i \rho \tau\right\} d \rho=\left\{\frac{2 \pi}{|d|}\right\}^{1 / 2} \exp \left\{\frac{i \tau^{2}}{2 d}+\frac{i \pi}{4} \operatorname{sgn} d\right\}
$$

where $d$ is a non-zero real and $\tau$ denotes the real dual variable of $\rho$. From this we conclude that

$$
\begin{aligned}
\mathscr{F}^{-1}\left[\exp \left\{\frac{i t}{2}\langle H(a) \eta, \eta\rangle\right\}\right](x) \\
=(2 \pi)^{-m} \int \exp \left\{\frac{i t}{2}\langle H(a) \eta, \eta\rangle-i\langle x, \eta\rangle\right\} d \eta \\
=\frac{(2 \pi|t|)^{-m}}{|\operatorname{det} H(a)|^{1 / 2}} \exp \left\{-\frac{i}{2 t}\left\langle H(a)^{-1} x, x\right\rangle\right. \\
\left.+\frac{i \pi}{4} \operatorname{sgn} H(a) \cdot \operatorname{sgn} t\right\} .
\end{aligned}
$$

Substituting $(4 \cdot 23)$ in $(4 \cdot 20)$ we obtain

$$
\begin{aligned}
f(t)= & \frac{(2 \pi)^{-m / 2}}{|\operatorname{Hess} h(a)|^{1 / 2}} \exp \left\{i t h(a)+\frac{i \pi}{4} \operatorname{sgn} H(a) \cdot \operatorname{sgn} t\right\} \\
& \times \int_{\mathbb{R}^{m}} \widehat{\hat{g}}_{1}(x) \exp \left\{-\frac{i}{2 t}\left\langle H(a)^{-1} x, x\right\rangle\right\} d x .
\end{aligned}
$$

9) $\mathscr{I}$ and $\mathscr{F}^{-1}$ denote respectively the Fourier transformation and the inverse Fourier transformation in the space of temperate distributions. 
Let us apply to the function $\phi(x)=\exp \left\{-\frac{i \operatorname{sgn} t}{2}\left\langle H(a)^{-1} x, x\right\rangle\right\}$ the Taylor's formula:

$$
\psi(x)=\sum_{|\alpha|<2 N} C_{\alpha} x^{\alpha}+\sum_{|\alpha|=2 N} x^{\alpha} \psi_{\alpha}(x),
$$

where

$$
C_{\alpha}=\left.\frac{1}{\alpha !}\left(\frac{\partial}{\partial x}\right)^{\alpha} \psi(x)\right|_{x=0}
$$

and

$$
\left|D_{x}^{\beta} \psi_{\alpha}(x)\right| \leq C_{2 N+\mid \beta, h, m} \sum_{|r| \leq 2 N+|\beta|} \sup _{x}\left|D^{r} \psi(x)\right| .
$$

Since $\exp \left\{-i\left\langle H(a)^{-1} x, x\right\rangle / 2 t\right\}=\exp \left\{-i \operatorname{sgn} t\left\langle H(a)^{-1} x /|t|^{1 / 2}, x /|t|^{1 / 2}\right\rangle / 2\right\}$, we obtain

$$
\begin{aligned}
\mid \int_{\mathbb{R}^{m}} \widehat{g}_{1}(x) & \exp \left\{-\frac{1}{2 t}\left\langle H(a)^{-1} x, x\right\rangle\right\} d x \\
& -\sum_{|\alpha|<2 N} C_{\alpha}|t|^{-|\alpha| / 2} \int_{\mathbb{R}^{m}} x^{\alpha} \widehat{g}_{1}(x) d x \mid \\
& \leq C_{2 N, h, m}|t|^{-(m / 2)-N} \sum_{|\alpha|=2 N} \int\left|x^{\alpha} \psi_{\alpha}\left(\frac{x}{\mid t^{\mid 1 / 2}}\right) \widehat{g}_{1}(x)\right| d x
\end{aligned}
$$

Now

$$
\left|\psi_{\alpha}\left(\frac{x}{|t|^{1 / 2}}\right)\right| \leq C_{|\alpha|, h, m}\left(1+\frac{|x|}{|t|^{1 / 2}}\right)^{|\alpha|} \leq C_{|\alpha|, h, m}(1+|x|)^{|\alpha|} \text { for } \quad|t| \geq 1 .
$$

Therefore

$$
\begin{aligned}
\sum_{|\alpha|=2 N} & \int_{\mathbf{R}^{m}}\left|x^{\alpha} \psi_{\alpha}\left(\frac{x}{|t|^{1 / 2}}\right) \widehat{g}_{1}(x)\right| d x \\
& \leq C_{2 N, h, m}^{\prime} \sum_{|r| \leq 4 N+m+1} \sup _{x}\left|x^{r} \widehat{g}_{1}(x)\right| \int_{\mathbf{R}^{m}} \frac{d x}{(1+|x|)^{m+1}} \\
& \leq C_{2 N, h, m} \sum_{|\gamma| \leq 4 N+m+1} \int_{B^{m}}\left|D^{r} g_{1}(\eta)\right| d \eta
\end{aligned}
$$

From $(4 \cdot 24),(4 \cdot 25),(4 \cdot 26)$ and the relation

$$
\int x^{\alpha} \hat{g}_{1}(x) d x=(2 \pi)^{m}\left(D^{\alpha} g_{1}\right)(0),
$$

we conclude that 


$$
\begin{aligned}
\mid f(t)- & (2 \pi)^{m / 2} \frac{\exp \left\{i t h(a)+\frac{i \pi}{4} \operatorname{sgn} H(a) \operatorname{sgn} t\right\}}{|\operatorname{Hess} h(a)|^{1 / 2}}|t|^{-m / 2} \\
& \times\left.\sum_{|\alpha|<2 N} C_{\alpha} D_{\eta}^{\alpha}\left[g(\varphi(\eta)) \frac{D(\varphi)}{D(\eta)}\right]\right|_{\eta=0}|t|^{|-| \alpha \mid / 2} \mid \\
& \leq C_{2 N, n, h, g}|t|^{-(m / 2)-N} \text { for }|t| \geq 1 .
\end{aligned}
$$

The following theorem we will use in $\S 7$ is concerned with the asymptotic behavior at infinity for a one-dimensional integral with a phase function of which a non-degenerate critical point is an end point of the integral.

Theorem 4. 2. Let $h(\xi)$ be a real-valued $C^{\infty}$ function defined in $[0, \infty)$ and $g \in C_{0}^{\infty}[0, \infty)$. Suppose that $h^{\prime}(0)=0, h^{\prime}(\xi) \neq 0$ for $\xi>0$ and $h^{\prime \prime}(0) \neq 0$. Then we have

$$
f(t)=\int_{0}^{\infty} g(\xi) \exp \{i t h(\xi)\} d \xi=\sum_{j=0}^{N-1} C_{j} t^{-(j+1) / 2}+R_{N}(t),
$$

and

$$
\left|R_{N}(t)\right| \leq C_{N, h, g} t^{-(N+1) / 2} \quad \text { for } \quad t \geq 1
$$

where

$$
\begin{gathered}
C_{j}=g_{1}^{(j)}(0)\left(\frac{\pi}{2}\right)^{1 / 2}\left(\frac{i}{h^{\prime \prime}(0)}\right)^{k} \frac{\exp \left\{i t h(0)+\frac{i \pi}{4} \operatorname{sgn} h^{\prime \prime}(0)\right\}}{\left|h^{\prime \prime}(0)\right|^{1 / 2} 2^{k} k !} \\
\text { for } j=2 k \text { even, } k=0,1,2, \cdots,
\end{gathered}
$$

and

$$
\begin{aligned}
& C_{j}=\frac{g_{1}^{(j)}(0)}{j !}\left(\frac{i}{h^{\prime \prime}(0)}\right)^{k+1} 2^{k} k ! \exp \{i t h(0)\} \\
& \text { for } \quad j=2 k+1 \text { odd }, k=0,1,2, \cdots .
\end{aligned}
$$

Proof. Since $h^{\prime}(\xi) \neq 0$ for $\xi>0$, it suffices to consider the case where the support of $g$ lies in a sufficiently small right neighborhood of 0 . Let $\xi=\varphi(\eta)$ be the substitution of variable such that $h(\varphi(\eta))-h(0)$ $=h^{\prime \prime}(0) \eta^{2} / 2$, which was defined in the proof of Theorem 4.1. Here 
$\eta=\left(2 \alpha(\xi) / h^{\prime \prime}(0)\right)^{1 / 2} \xi$ and $\alpha(\xi)=\int_{0}^{1}(1-\rho) h^{\prime \prime}(\xi \rho) d \rho$. Put $g_{1}(\hat{\xi})=g(\varphi(\eta))$ $<D(\varphi) / D(\eta)$. Choose an even function $\phi \in C_{0}^{\alpha}(-\infty, \infty)$ so that $\phi=1$ near supp $g_{1}$. Then we have

$$
f(t)=\exp \{i l h(0)\} \int_{0}^{\infty} \phi(\eta) g_{1}(\eta) \exp \left\{i t h^{\prime \prime}(0) \eta^{2} / 2\right\} d \eta
$$

Substituting the Taylor formula for $g_{1}$ :

$$
g_{1}(\eta)=\sum_{j=0}^{N-1} \frac{g_{1}^{(j)}(0)}{j !} \eta^{j}+\eta^{N} g_{1, N}(\eta), \quad g_{1, N} \in C^{(\lrcorner}[0, \infty)
$$

in the above integral, we get

$$
\begin{aligned}
& f(t) \exp \{-i t h(0)\}=g(0) \int_{0}^{\infty} \phi(\eta) \operatorname{cxp}\left\{i t h^{\prime \prime}(0) \cdot \eta^{2} / 2\right\} d \eta \\
& +\sum_{j=1}^{N-1} \frac{g_{1}^{(j)}(0)}{j !} \int_{0}^{\omega} \eta \eta^{j} \phi(\eta) \exp \left\{i \iota h^{\prime \prime}(0) \eta^{2} / 2\right\} d \eta \\
& +\int_{0}^{\omega} \eta^{N} g_{1, N}(\eta) \phi(\eta) \exp \left\{i t h^{\prime \prime}(0) \eta^{2} / 2\right\} d \eta \\
& =f_{0}(t)+\sum_{j=1}^{N-1} g_{1}^{(j)}(0) f_{j}(l) / j !+\int_{N}(t), \text { respectively. }
\end{aligned}
$$

First consider $f_{\circ}(t)$. Since $\phi(\eta)$ is an even function,

$$
f_{\circ}(t)=\frac{g(0)}{2} \int_{-\infty}^{\omega} \phi(\eta) \exp \left\{i t h^{\prime \prime}(0) \eta \eta^{2} / 2\right\} d \eta
$$

Let us apply $2^{\circ}$ of Theorem 4.1 for $m=1$ to this integral. Since $\phi^{(j)}(0)=0$ for $j \geq 1$, we have

$$
\begin{gathered}
f_{\circ}(t)=\left(\frac{\pi}{2}\right)^{1 / 2} \frac{g(0) \exp \left\{i \pi \operatorname{sgn} h^{\prime \prime}(0) / 4\right\}}{\left|h^{\prime \prime}(0)\right|^{1 / 2}} t^{-1 / 2}+O\left(t^{-\omega}\right) \\
\text { as } t \rightarrow \infty .
\end{gathered}
$$

Next consider $f_{1}(t)$. Using the relation:

$(4 \cdot 33) \quad \eta \exp \left\{i l h^{\prime \prime}(0) \eta^{\prime \prime} / 2\right\}=\frac{1}{i t h^{\prime \prime}(0)} \frac{d}{d \eta}\left(\exp \left\{i t h^{\prime \prime}(0) \eta^{2} / 2\right\}\right)$

and integrating by parts, we get

$$
f_{1}(t)=-\frac{1}{i t h^{\prime \prime}(0)}-\frac{1}{i t h^{\prime \prime}(0)} \int_{0}^{\phi^{\prime}} \phi^{\prime}(\eta) \exp \left\{i t h^{\prime \prime}(0) \eta^{2} / 2\right\} d \eta
$$


Since $\phi^{\prime}(\eta) \in C_{0}^{\infty}(0, \infty)$, the second term of the right hand side decreases faster than any negative power of $t$ as $t \rightarrow \infty$. Repeating the same argument we obtain for $k=0,1,2, \cdots$

$$
\begin{aligned}
f_{2 k+1}(t) & =\int_{0}^{\infty} \eta^{2 k+1} \phi(\eta) \exp \left\{i t h^{\prime \prime}(0) \eta^{2} / 2\right\} d \eta \\
& =\left(i / h^{\prime \prime}(0)\right)^{k+1} 2^{k} k ! t^{-k-1}+O\left(t^{-\infty}\right) \text { as } \quad t \rightarrow \infty .
\end{aligned}
$$

On the other hand, consider $f_{2}(t)$. Integrating by parts, we find

$$
\begin{aligned}
f_{2}(t) & =-\frac{1}{i t h^{\prime \prime}(0)} \int_{0}^{\infty}\left(\phi(\eta)+\eta \phi^{\prime}(\eta)\right) \exp \left\{i t h^{\prime \prime}(0) \eta^{2} / 2\right\} d \eta \\
& =\frac{i}{t h^{\prime \prime}(0)} \int_{0}^{a} \phi(\eta) \exp \left\{i t h^{\prime \prime}(0) \eta^{2} / 2\right\} d \eta+O\left(t^{-\infty}\right) \text { as } t \rightarrow \infty
\end{aligned}
$$

In view of $(4 \cdot 32)$, we have

$$
f_{2}(t)=\left(\frac{\pi}{2}\right)^{1 / 2} \frac{i \exp \left\{i \pi \operatorname{sgn} h^{\prime \prime}(0) / 4\right\}}{h^{\prime \prime}(0)\left|h^{\prime \prime}(0)\right|^{1 / 2}} t^{-3 / 2}+O\left(t^{-\infty}\right) \quad \text { as } \quad t \rightarrow \infty .
$$

Proceeding inductively, we deduce

$$
\begin{aligned}
f_{2 k}(t) & =\left(\frac{\pi}{2}\right)^{1 / 2}\left(\frac{i}{h^{\prime \prime}(0)}\right)^{k} \frac{(2 k) !}{\left|h^{\prime \prime}(0)\right|^{1 / 2} 2^{k} k !} \exp \left\{i \pi \operatorname{sgn} h^{\prime \prime}(0) / 4\right\} \\
& \times t^{-(2 k+1) / 2}+O\left(t^{-\infty}\right) \quad \text { as } \quad t \rightarrow \infty, k=0,1,2, \cdots
\end{aligned}
$$

By successive integrations by parts, we also find that

$$
f_{N}(t)=O\left(t^{-\{N+1) / 2}\right) \quad \text { as } \quad t \rightarrow \infty .
$$

From $(4 \cdot 31),(4 \cdot 34),(4 \cdot 35)$ and $(4 \cdot 36)$, we conclude $(4 \cdot 27),(4 \cdot 28)$, $(4 \cdot 29)$ and $(4 \cdot 30)$.

Q.E.D.

\section{$\S 5$. Asymptotic Behavior at Infinity of the Fourier Transform of a Measure Concertrated on a Hypersurface}

The method of stationary phase is applied to the investigation of the asymptotic behavior at infinity of the Fourier transforms of measures with smooth density concentrated on smooth hypersurfaces. In doing this, we must recall some basic notions and theorems on hypersurfaces in differential geometry. 
By a $C^{\infty}$ hypersurface $S$ in $\boldsymbol{E}^{n}$ we mean an $(n-1)$-dimensional $C^{\omega}$ manifold without boundary embedded in $\boldsymbol{\Xi}^{n}$. We shall denote by $s$ the moving point on $S$. We can assign a unit normal $\boldsymbol{n}(s)$ to each point in a coordinate neighborhood of a point on $S$ in a such a way that $n(s)$ is a $C^{\omega}$ vector field. But this can be done globally on $S$ if and only if $S$ is orientable. Let $n(s)$ be a $C^{\infty}$ unit normal vector field on $S$. Then the $n(s)$ defines a $C^{\omega}$ map of $S$ into the unit $(n-1)$-sphere $\Omega$ and this map is called the spherical or Gauss map. Our present study is local so we fix a $C^{\infty}$ unit normal vector field on a neighborhood $S^{*}$ of a point on $S$ in question. For any $s$ in $S^{*}$ and any vector $v$ in the tangent space $T S_{s}$, define the linear map $L_{s}: T S_{s} \rightarrow T S_{s}$ in the following way. Pick any curve $s=s(t)$ through $s$ so that $d s(t) /\left.d t\right|_{t=0}=v$. We follow the normal $n=n(s(t))$ as $s$ traverses the curve. Then $d \boldsymbol{n}(s(t)) /\left.d t\right|_{t=0}$ $\in T S_{s}$ and this vector is independent of the choice of the curve $s=s(t)$ so long as it has the prescribed tangent $v$ at $t=0$, so we define $L_{s} v$ $=d \boldsymbol{n}(s(t)) /\left.d t\right|_{t=0}$. This linear map $L_{s}$ in the tangent space $T S_{s}$ is called the Weingarten map. It is easy to see that $L_{s}$ is self-adjoint with respect to the inner product in $T S_{s}$ induced naturally from $\mathbb{B}^{n}$ so the representation matrix of $L_{s}$ with respect to an orthogonal base of $T S_{s}$ is symmetric. For each integer $k \geq 1,\left\langle L_{s}{ }^{k-1} v, w\right\rangle$, $v, w \in T S_{s}$ is called the $k$-th fundamental form on $S$. The algebraic invariants of the linear map $L_{s}$ at each point define the embedded geometric invariants of $S$ at each point. Thus the determinant of $L_{s}$ is the Gaussian or total curvature $K(s)$ of $S$ at $s$, the trace of $L_{s} /(n-1)$ is the mean curvature. Since $L_{s}$ is self-adjoint, its eigenvalues are all real and they are called principal curvatures at $s$. If they are all distinct, then the corresponding eigenvectors of unit length are determined up to a sign and they are called the principal directions at $s$. We have defined making use of $\boldsymbol{n}(s)$ and $L_{s}$ the Gaussian curvature of the hypersurface at each point. However, if $n$ is odd, the Gaussian curvature depends only on the metric in the tangent space $T S_{s}$, that is, only on the Riemannian metric on $S$ induced from $\boldsymbol{\Xi}^{n}$ (Gauss' theorema egregium). Thus the Gaussian curvature is, if $n$ is odd, an intrinsic invariant that is independent of the embedding (i.e., of $n(s)$ and $L_{s}$ ). But this is false for any even $n$. Note that the principal curvatures change their sign whether $n$ is odd 
or even if we replace $n(s)$ by $-n(s)$. Here we give the formula for the Gaussian curvature $K(s)$ at each point $s$ of a $C^{\infty}$ hypersurface defined by an equation $s_{n}=h\left(s^{\prime}\right)$ where $s^{\prime}$ denotes $\left(s_{1}, \cdots, s_{n-1}\right)$. We choose the $C^{\infty}$ unit normal vector field $\boldsymbol{n}(s)$ such that $\langle\boldsymbol{n}(s),(0, \cdots, 0,1)\rangle$ $>0$, that is,

$$
\boldsymbol{n}(s)=\left\{1+\left|\underset{s^{\prime}}{\operatorname{grad}} h\left(s^{\prime}\right)\right|^{2}\right\}^{-1 / 2}\left(-\frac{\partial h}{\partial s_{1}}\left(s^{\prime}\right), \cdots,-\frac{\partial h}{\partial s_{n-1}}\left(s^{\prime}\right), 1\right) .
$$

Then the Gaussian curvature $K(s)$ with respect to $\boldsymbol{n}(s)$ is given in the form

$$
K(s)=\frac{(-1)^{n-1} \operatorname{det}\left(\frac{\partial^{2} h}{\partial s_{j} \partial s_{k}}\left(s^{\prime}\right)\right)}{\left\{1+\mid \underset{s^{\prime}}{\left.\left.\operatorname{grad} h\left(s^{\prime}\right)\right|^{2}\right\}^{(n+1) / 2}}\right.} .
$$

Hence we have $K(s)=(-1)^{n-1} \operatorname{Hess} h\left(s^{\prime}\right)$ if $s^{\prime}$ is a critical point of $h\left(s^{\prime}\right)$. Let $\lambda_{1}, \cdots, \lambda_{n-1}$ be the eigenvalues of the Hessian matrix $\left(\partial^{2} h\left(s^{\prime}\right) / \partial s_{j} \partial s_{k}\right)$. Then the principal curvatures in the normal direction $n(s)$ are $-\lambda_{1}, \cdots,-\lambda_{n-1}$.

Both the Gaussian curvature and the Weingarten operator are closely related to the convexity of a hypersurface. A hypersurface $S$ in $\boldsymbol{\sigma}^{n}$ is said to be convex at a point $s \in S$ if the hyperplane $\mathcal{H}_{s}$ of $\mathbb{F}^{n}$ tangent to $S$ at $s$ does not separate a neighborhood of $s$ in $S$ into two parts. Moreover, if $s$ is the only point of a neighborhood which lies on $\mathcal{H}_{s}$, then $S$ is said to be strictly convex at $s$. If, for every $s \in S, \mathcal{H}_{s}$ does not separate $S$ into two parts, then $S$ is said to be convex. If, for every $s \in S, s$ is the only point of $S$ which lies on $\mathcal{H}_{s}, S$ is said to be strictly convex. A convex hypersurface is always orientable. Convexity of a $C^{\infty}$ hypersurface $S$ can be expressed in terms of a definiteness condition on the Weingarten operator $L_{s}$. A $C^{\infty}$ hypersurface $S$ in $g^{n}$ is strictly convex at a point $s$ if $L_{s}$ is definite, i.e. if the second fundamental form $\left\langle L_{s} v, v\right\rangle$ is either positive or negative definite. The converse is not true. $L_{s}$ may be semi-definite and therefore the Gaussian curvature $K(s)$ may vanish. A $C^{\infty}$ hypersurface $S$ is convex at a point $s$ if and only if $L_{s}$ is semi-definite.

Now we recall the following theorems concerning global property. 
Theorem D.G. I. For a $C^{\circ}$ closed hypersurface in $\mathbb{G}^{n}(n \geq 2)$, namely a connected and compact $(n-1)$-dimensional $C^{\circ}$ manifold reithout boundary embedded in $\mathbf{\Omega}^{n}$, the following conditions are equivalent.

(1) The Gaussian curvature $K(s)$ of $S$ never vanishes on $S$.

(2) The Weingarten operator $L_{s}$ is definite for every $s \in S$, that $i s$, the second fundamental form $\left\langle L_{s} v, v\right\rangle$ is definite everywhere on $S$.

(3) $S$ is orientable and the spherical map $S \rightarrow \Omega$ is a $C^{\mathrm{c}}$ diffeomorphism.

Theorem D.G. Il (Hadamard). Any one of the conditions above implies that $S$ is strictly convex.

Remark. If we mean by a hypersurface in $\mathbb{E}^{n}$ an $(n-1)$-dimensional manifold with an immersion, the above theorems are true only for $n \geq 3$.

For proofs of these theorems and more details concerning curvatures of hypersurfaces, one may consult, for example Flanders [3], Hicks [6], Kobayashi and Nomizu [9] or Sternberg [20].

Under these preparations, we proceed to the following theorem which is our main purpose in this section. This theorem which describes the asymptotic behavior at infinity of the Fourier transforms of the measures with $C^{\circ}$ density concertrated on $C^{\infty}$ hypersurfaces in $\mathbb{E}^{n}$, has been investigated by many authors, e.g. Fedoriuk [थ], Grusǐn [5], Littman [10], Vainberg [23] and others (see also Matsumura [1 1007 , [14], [15]).

Theorem 5. 1. Lel $S$ be a $C^{m}$ hypersurface in $\mathbb{E}^{n}$, "! a $C^{m}$ function defined on $S$ reith compact support and define

$$
I(x)=\int_{S} \mu(s) \exp \{i\langle x, s\rangle\} d S, \quad x \in \mathbb{R}^{n},
$$

where dS is the surface element on $S$. Assume that the Gaussian curvature $K(s)$ of $S$ does not vanish on supp ". Then the set of points on supp $\mu$ at which the normal to $S$ is parallel to $\theta$ is finite in number for each unit vector $\theta \in \Omega .^{10)} \quad W e$ denote these points by $s^{1}(\theta), \cdots, s^{r}(\theta)$.

10) $\theta$ and $\Omega$ denote respectively a unit vector and the unit sphere in $\mathscr{E}^{n}$ and in $\mathbb{R}^{n}$. 
Taking $\theta$ as the positive direction at $s^{l}(\theta)$, denote by $p_{l}{ }^{+}(\theta)$ and $p_{l}{ }^{-}(\theta)$ the number of positive and negative principal curvatures at $s^{l}(\theta)$ respectively. Then the asymptotic behavior of $I(x)$ for $|x| \rightarrow \infty$ along the ray $x=|x| \theta$, is given by

$$
\begin{aligned}
I(x)= & (2 \pi)^{(n-1) / 2} \sum_{l=1}^{r}\left[\frac { \mu ( s ^ { l } ( \theta ) ) } { | K ( s ^ { l } ( \theta ) ) | ^ { 1 / 2 } } \operatorname { e x p } \left\{i|x|\left\langle\theta, s^{l}(\theta)\right\rangle\right.\right. \\
& \left.\left.+\frac{i \pi}{4}\left(p_{l}{ }^{-}(\theta)-p_{l}{ }^{+}(\theta)\right)\right\}\right]|x|^{-(n-1) / 2}+q(x),
\end{aligned}
$$

where for each multi-index ע

$$
(\partial / \partial x)^{\nu} q(x)=O\left(|x|^{-(n+1) / 2}\right) \text { as }|x| \rightarrow \infty,
$$

uniformly for $\theta \in \Omega$.

Proof. We first remark that the measure $m_{\|}$with $C^{\infty}$ density $\mu(s)$ concentrated on $S$ is defined by

$$
\left\langle m_{\mu}, \varphi\right\rangle=\int_{S} \mu(s) \varphi(s) d S, \quad \varphi \in \mathscr{S}\left(\Xi^{n}\right)
$$

It is obvious that supp $m_{\mu} \subset S$. Since the Fourier transform of $m_{\mu}$ is defined by

$$
\left\langle\mathscr{F}^{\prime} m_{\mu}, \psi\right\rangle=\left\langle m_{\mu}, \mathscr{F} \psi\right\rangle, \quad \psi \in \mathscr{S}\left(\mathbb{R}^{n}\right),
$$

we have

$$
\begin{aligned}
\left\langle\mathscr{I} m_{\mu}, \psi\right\rangle & =\int_{S} \mu(s)\left\{\int_{\mathbb{R}^{n}} \psi(x) \exp \{-i\langle x, s\rangle\} d x\right\} d S \\
& =\int_{\mathbb{R}^{n}}\left\{\int_{S} \mu(s) \exp \{-i\langle x, s\rangle\} d S\right\} \psi(x) d x .
\end{aligned}
$$

Thus $I(-x)$ is the Fourier transform of $m_{\mu}$. Let us return to the study of the asymptotic behavior of $I(x)$ as $|x| \rightarrow \infty$. First we will study the case when $x$ is $\left(0, \cdots, 0, x_{n}\right)$ and write simply $I\left(x_{n}\right)$ instead of $I(0, \cdots$, $\left.0, x_{n}\right)$. We show that the major contribution to $I\left(x_{n}\right)$ as $\left|x_{n}\right| \rightarrow \infty$ arises from the immediate vicinity of the points at which the normal to the surface $S$ is parallel to the vector $(0, \cdots, 0,1)$. For this let us take a finite number of sufficiently small patchs $\left\{U_{j}\right\}$ for the $C^{\infty}$ manifold $S$ which cover supp $\mu$ and a $C^{\infty}$ partition of unity $\left\{\varphi_{j}\right\}$ subordinate to 
this covering such that $\sum \varphi_{j}(s)=1$ on supp $\mu$. Decompose

$$
I\left(x_{n}\right)=\sum_{j} \int_{U_{j}} \mu_{j}(s) \exp \left\{i x_{n} s_{n}\right\} d S \equiv \sum_{j} I_{j}\left(x_{n}\right), \text { respectively, }
$$

where $\iota_{j}=\varphi_{j} / l$. We consider a patch $U_{j}$ which does not contain any point such that the normal to $S$ at this point is parallel to the vector $(0, \cdots, 0,1)$. Since $U_{j}$ is a part of a $C^{-\jmath}$ hypersurface, $U_{j}$ inay be represented by the equations $s_{k}=s_{k}\left(\sigma_{1}, \cdots, \sigma_{n-1}\right), k=1, \cdots, n$ where the $s_{k}$. are $C^{\infty}$ functions defined on some open set in $\sigma^{\prime}=\left(\sigma_{1}, \cdots, \sigma_{n-1}\right)$-space. Then our above assumption on $U_{j}$ implies that there exists at least a tangent vector which is not orthogonal to the vector $(0, \cdots, 0,1)$. Thus we have $\partial s_{n}\left(\sigma^{\prime}\right) / \partial \sigma_{i} \neq 0$ for some $i$. By changing from integration over $U_{j}$ to integration with respect to $\sigma_{1}, \cdots, \sigma_{n-1}$, we have

$$
I_{j}\left(x_{n}\right)=\int \mu_{i}\left(s\left(\sigma^{\prime}\right)\right) \omega\left(\sigma^{\prime}\right) \exp \left\{i x_{n} s\left(\sigma^{\prime}\right)\right\} d \sigma^{\prime},
$$

where $\omega\left(\sigma^{\prime}\right) d \sigma^{\prime}=d S=\left\{\sum_{k=1}^{n}\left|D\left(s_{1}, \cdots, \hat{s}_{k}, \cdots, s_{n}\right) / D\left(\sigma_{1}, \cdots, \sigma_{n-1}\right)\right|^{2}\right\}^{1 / 2} d \sigma_{1} \cdots d \sigma_{n-1}$ ( means "omit"). Since $\underset{\sigma^{\prime}}{\operatorname{grad}} s_{n}\left(\sigma^{\prime}\right) \neq 0$ on $\operatorname{supp}_{\sigma^{\prime}} \|_{i}\left(s\left(\sigma^{\prime}\right)\right)$, we have by $1^{\circ}$ in Theorem 4.1 that

$$
I_{j}\left(x_{n}\right)=O\left(\left|x_{n}\right|^{-\infty}\right) \quad \text { as } \quad\left|x_{n}\right| \rightarrow \infty .
$$

From this, we see that the main contribution to $I\left(x_{n}\right)$ as $\left|x_{n}\right| \rightarrow \infty$ arises from those terms $I_{j}\left(x_{n}\right)$ such that $U_{j}$ contains points at which the normal to $S$ is parallel to $(0, \cdots, 0,1)$. Such $U_{j}$ may be represented by the equation in the form $s_{n}=h\left(s_{1}, \cdots, s_{n-1}\right)$ where $h$ is a $C^{n}$ function. In fact, let $s_{k}=s_{k}\left(\sigma_{1}, \cdots, \sigma_{n-1}\right), k=1, \cdots, n$ be the system of equations that defines $U_{j}$ and $s(\theta)$ a point in $U_{j}$ at which the normal to $S$ is parallel to $\theta=(0, \cdots, 0,1)$. Then the unit normal at $s(\theta)$ is given in the form

$$
\begin{gathered}
C\left(-\frac{D\left(s_{2}, \cdots, s_{n}\right)}{D\left(\sigma_{1}, \cdots, \sigma_{n-1}\right)}, \cdots,(-1)^{k} \frac{D\left(s_{1}, \cdots, \widehat{s}_{k}, \cdots, s_{n}\right)}{D\left(\sigma_{1}, \cdots, \sigma_{n-1}\right)},\right. \\
\left.\cdots,(-1)^{n} \frac{D\left(s_{1}, \cdots, s_{n-1}\right)}{D\left(\sigma_{1}, \cdots, \sigma_{n-1}\right)}\right)\left.\right|_{\sigma^{\prime}=\sigma^{\prime}(\theta)},
\end{gathered}
$$

where $C=\left\{\sum_{k=1}^{n}\left|D\left(s_{1}, \cdots, \widehat{s}_{k}, \cdots, s_{n}\right) / D\left(\sigma_{1}, \cdots, \sigma_{n-1}\right)\right|^{2}\right\}^{-1 / 2}$ and $s(\theta)=s\left(\sigma^{\prime}(\theta)\right)$. Since this normal is, by assumption, parallel to $\theta=(0, \cdots, 0,1)$, we have $D\left(s_{1}, \cdots, s_{n-1}\right) /\left.D\left(\sigma_{1}, \cdots, \sigma_{n-1}\right)\right|_{\sigma^{\prime}=\sigma^{\prime}(\theta)} \neq 0$. Consequently we can solve, by the inverse function theorem, as $\sigma_{k}=\sigma_{k}\left(s_{1}, \cdots, s_{n-1}\right)$ where the $\sigma_{k}$ are $C^{\text {? }}$ 
functions of $s^{\prime}=\left(s_{1}, \cdots, s_{n-1}\right)$. By substituting this in $s_{n}=s_{n}\left(\sigma_{1}, \cdots, \sigma_{n-1}\right)$ we see that $U_{j}$ is represented by the equation $s_{n}=s_{n}\left(\sigma_{1}\left(s^{\prime}\right), \cdots, \sigma_{n-1}\left(s^{\prime}\right)\right)$ $\equiv h\left(s_{1}, \cdots, s_{n-1}\right)$. Further the vector $\left(-\partial h\left(s^{\prime}\right) / \partial s_{1}, \cdots,-\partial h\left(s^{\prime}\right) / \partial s_{n-1}\right.$, 1) $\left.\right|_{s^{\prime}=s^{\prime}(\theta)}$ is normal to $S$ at the point $s(\theta)$ and therefore parallel to $(0, \cdots, 0,1)$ by assumption. Thus we have $\underset{s^{\prime}}{\operatorname{grad}} h\left(s^{\prime}(\theta)\right)=0$, that is, $s^{\prime}(\theta)$ is a critical point of the function $h\left(s^{\prime}\right)$. From the assumption that the Gaussian curvature $K(s)$ of $S$ does not vanish on supp $\mu$, it follows that the critical point $s^{\prime}(0)$ is non-degenerate. For we have Hess $h\left(s^{\prime}(\theta)\right) \neq 0$ according to $(5 \cdot 2)$. Therefore the points at which the normal to $S$ is parallel to $(0, \cdots, 0,1)$ are isolated. So we may assume that $U_{j}$ will contain no more than one such point. If we denote by $\lambda_{1}, \cdots, \lambda_{n-1}$ the eigenvalues of the Hessian matrix $H\left(s^{\prime}(\theta)\right)$ $=\left(\partial^{2} h\left(s^{\prime}(\theta)\right) / \partial s_{j} \partial s_{k}\right)$, the principal curvatures of $S$ at $s(\theta)$ with respect to the unit normal vector field $(5 \cdot 1)$ are given by $-\lambda_{1}, \cdots,-\lambda_{n-1}$ and we have $\sum_{j=1}^{n-1} \operatorname{sgn} \lambda_{j}=p^{-}(\theta)-p^{+}(\theta)$. Let us return to the integral $I_{j}\left(x_{n}\right)$. By changing from integration over $U_{j}$ to integration with respect to $s^{\prime}$, we have

$$
I_{j}\left(x_{n}\right)=\int \mu\left(s^{\prime}, h\left(s^{\prime}\right)\right)\left\{1+\left.\underset{s^{\prime}}{\operatorname{grad}} h\left(s^{\prime}\right)\right|^{2}\right\}^{1 / 2} \exp \left\{i x_{n} h\left(s^{\prime}\right)\right\} d s^{\prime} .
$$

From the above consideration, it is possible to apply the case $2^{\circ}$ of Theorem 4.1 to this integral. Thus we get the following asymptotic formula :

$$
\begin{aligned}
I_{i}\left(x_{n}\right)= & (2 \pi)^{(n-1) / 2} \frac{\mu_{j}(s(0))}{|K(s(\theta))|^{1 / 2}} \exp \left\{i x_{n} h\left(s^{\prime}(\theta)\right)\right. \\
& \left.+\frac{i \pi}{4}\left(p^{-}(\theta)-p^{+}(\theta)\right) \operatorname{sgn} x_{n}\right\}\left|x_{n}\right|^{-(n-1) / 2} \\
& +O\left(\left|x_{n}\right|^{-(n+1) / 2}\right) \quad \text { as } \quad\left|x_{n}\right| \rightarrow \infty .
\end{aligned}
$$

The general case is easily reduced to this case. In fact, let $T$ be an orthogonal transformation in $\boldsymbol{G}^{n}$ such that ${ }^{t} T \theta={ }^{i}(0, \cdots, 0,1)$. By making the change of variables $s=T \eta$, we have

$$
\begin{aligned}
I_{j}(x) & =\int_{U_{j}} \mu_{j}(T \eta) \exp \left\{i\left\langle x,{ }^{t} T \eta\right\rangle\right\} d S_{\eta} \\
& =\int_{U_{j}} \mu_{j}(T \eta) \exp \left\{i|x| \eta_{\eta}\right\} d S_{\eta} .
\end{aligned}
$$


After applying (5.9) to this integral, we perform the inverse transformation $\eta=T^{-1} s$. Then we obtain

$$
\begin{aligned}
& I_{j}(x)=(2 \pi)^{(n-1) / 2} \frac{\mu(s(\theta))}{|K(s(\theta))|^{1 / 2}} \exp \{i|x|\langle\theta, s(\theta)\rangle \\
&\left.+\frac{i \pi}{4}\left(p^{-}(0)-p^{+}(\theta)\right)\right\}|x|^{-(n-1) /^{2}}+O\left(|x|^{-(n+1) / 2}\right) \\
& \text { as }|x| \rightarrow \infty
\end{aligned}
$$

along the ray $x=|x| \theta$. Further it is easy to see that the order relation $O$ is uniform with respect to $\theta \in \Omega$. Summing up, we have

$$
I(x)=\sum_{l=1}^{r} C_{l}(\theta) \exp \left\{i|x|\left\langle\theta, s^{l}(\theta)\right\rangle\right\}|x|^{-(n-1) / 2}+O\left(|x|^{-(n+1) / 2}\right)
$$

for $|x| \rightarrow \infty$, uniformly for $\theta=x /|x| \in \Omega$ where

$$
C_{l}(\theta)=(2 \pi)^{(n-1) / 2} \frac{\mu\left(s^{l}(\theta)\right)}{\left|K\left(s^{l}(\theta)\right)\right|^{1 / 2}} \exp \left\{\frac{i \pi}{4}\left(p^{-}(\theta)-p^{+}(\theta)\right)\right\} .
$$

Set

$$
q(x)=I(x)-\sum_{l=1}^{r} C_{l}(\theta) \exp \left\{i \mid x i\left\langle\theta, s^{l}(\theta)\right\rangle\right\}|x|^{-(n-1) / 2} .
$$

Noting that 0 is a function of $x$, differentiate the both sides.

$$
\begin{aligned}
(\partial / \partial x)^{\nu} q(x)=(\partial / \partial x)^{\nu} I(x) \\
\quad-\sum_{l=1}^{r}\left(i s^{l}(\theta)\right)^{\nu} C_{l}(\theta) \exp \left\{i|x|\left\langle\theta, s^{l}(\theta)\right\rangle\right\} \\
\quad \times|x|^{-(n-1) / 2}+O\left(|x|^{-(n+1) / 2}\right) \quad \text { as } \quad|x| \rightarrow \infty .
\end{aligned}
$$

Applying the asymptotic formula obtained above to the integral

$$
(\partial / \partial x)^{\nu} I(x)=\int_{S}(i s)^{\nu} / t(s) \exp \{i\langle x, s\rangle\} d S,
$$

we have

$(\partial / \partial x)^{\nu} q(x)=O\left(|x|^{-(n+1) / 2}\right)$ for $|x| \rightarrow \infty$, uniformly for $\theta=x /|x| \in \Omega$.

This completes the proof.

In particular, consider the case when $S$ is a closed $C^{\infty}$ hypersurface. As stated before, if the Gaussian curvature $K(s)$ does not vanish on $S$, 
$S$ is strictly convex and the Gauss map is a diffeomorphism of $S$ to $\Omega$. We choose as $(s)$ the outward unit normal vector field and denote by $s(\theta)$ the inverse map of the Gauss map $S \ni s \mapsto n(s) \in \Omega$. Then, for each $\theta \in \Omega$, there exist only two points $s(\theta)$ and $s(-\theta)$ at which the normal to $S$ is parallel to $\theta$. The $n-1$ principal curvatures of $S$ at $s(\theta)$ and at $s(-\theta)$ with respect to the direction $\theta$ are all positive and all negative respectively. In this case we have the following asymptotic formula:

$$
\begin{aligned}
I(x)= & \int_{S} \mu(s) \exp \{i\langle x, s\rangle\} d S \\
= & (2 \pi)^{(n-1) / 2}\left[\frac{\mu(s(\theta))}{|K(s(\theta))|^{1 / 2}} \exp \left\{i|x|\langle\theta, s(\theta)\rangle-\frac{i \pi}{4}(n-1)\right\}\right. \\
& \left.+\frac{\mu(s(-\theta))}{|K(s(-\theta))|^{1 / 2}} \exp \left\{i|x|\langle\theta, s(-\theta)\rangle+\frac{i \pi}{4}(n-1)\right\}\right] \\
& \times|x|^{-(n-1) / 2}+q(x)
\end{aligned}
$$

as $|x| \rightarrow \infty$ along the ray $x=|x| \theta$, where $q(x)$ satisfies $(5 \cdot 5)$. If $S$ is the unit sphere $\Omega$ of ${ }^{n}$ and $\mu(s) \equiv 1$, we have the well known formula

$$
\begin{aligned}
I(x)= & \int_{\Omega} \exp \{i\langle x, s\rangle\} d S=2 \pi^{n / 2}(|x| / 2)^{-(n-2) / 2} J_{(n-2) / 2}(|x|) \\
= & 2 \pi^{(n-1) / 2}(|x| / 2)^{-(n-1) / 2} \cos \left(|x|-\frac{\pi}{4}(n-1)\right) \\
& +O\left(|x|^{-(n+1) / 2}\right)
\end{aligned}
$$

as $|x| \rightarrow \infty$, where $J_{\nu}(t)$ denotes the Bessel function of order $\nu$.

\section{§ 6. Basie Formulas}

Let $\lambda(\xi)$ be a real valued function on 思 $^{n}$ satisfying the conditions:

( $\lambda$. i) $\lambda(\xi)$ is positively homogeneous of degree 1 .

( $\lambda$. ii) $\lambda(\xi)$ is positive and $C^{\infty}$ in $\xi^{n} \backslash\{0\}$.

Then the set $S=\left\{s \in \mathbb{G}^{n} ; \lambda(s)=1\right\}$ forms a $C^{\infty}$, closed and non-singular surface of dimension $n-1$ which encloses the origin. For we have $\operatorname{grad} \lambda(\xi) \neq 0$ for $\xi \in \operatorname{s}^{n} \backslash\{0\}$ from the Euler's relation $\sum_{j=1}^{n} \xi_{j} \partial \lambda(\xi) / \partial \xi_{j}$ $=\lambda(\xi)$. From this we see that $S$ is non-singular, namely a $C^{\infty}$ manifold of dimension $n-1$. Since the map $\Omega \ni \xi \mapsto \xi / \lambda(\xi) \in S$ is one to one and bicontinuous, $S$ is a closed surface and encloses the origin. 
Making use of the function $\lambda(\xi)$, we can introduce new coordinates $(\rho, s)$ in the space $\mathbb{S}^{n} \backslash\{0\}$ such that

$$
\xi=\rho s, \quad 0<\rho<+(x), \quad s \in S .
$$

Then we have

$$
d \xi=\left(\rho^{n-1} /|\operatorname{grad} \lambda(s)|\right) d \rho d S .
$$

Take a finite covering of $S$ which consists of sufficiently small open subsets of $S$, and a $C^{\circ}$ partition of unity $\left\{\Theta_{j}(s)\right\}$ subordinate to this covering. If we extend these functions to the space $\mathbb{R}^{n} \backslash\{0\}$ by positive homogeneity of degree $0: \Theta_{i}(\xi)=\Theta_{i}(\xi / \lambda(\xi)),\left\{\Theta_{i}(\xi)\right\}$ is a $C^{\infty}$ partition of unity in $\mathbb{E}^{\prime \prime} \backslash\{0\}$. Then it suffices to show

$$
\Theta_{i}(\xi) d \xi=\Theta_{i}(s)\left(\rho^{n-1} /|\operatorname{grad} \lambda(s)|\right) d \rho d S .
$$

Let $s^{\circ}$ be a point in $\operatorname{supp} \Theta_{i}(s) \subset S$. From the assumption i) we may assume without loss of generality that $\partial \lambda(s) / \partial \xi_{n} \neq 0$ in a small neigh. borhood of $s^{\circ}$ on $S$. By the implicit function theorem, there exists a $C^{\infty}$ function $s_{n}=h\left(s^{\prime}\right), s^{\prime}=\left(s_{1}, \cdots, s_{n-1}\right)$ such that $\lambda\left(s^{\prime}, h\left(s^{\prime}\right)\right) \equiv 1$ and $\partial h\left(s^{\prime}\right) / \partial s_{j}$ $=-\left(\partial \lambda(s) / \partial \xi_{j}\right) /\left(\partial \lambda(s) / \partial \xi_{n}\right), j=1, \cdots, n-1$. In a conic neighborhood of $s^{\circ}$ which contains $\operatorname{supp}_{\hat{\xi}} \Theta_{i}(\xi)$, we have

$$
\begin{aligned}
& \frac{D\left(\xi_{1}, \xi_{2}, \cdots, \xi_{n}\right)}{D D\left(\rho, s_{1}, \cdots, s_{n-1}\right)}=(-1)^{n} \rho^{n-1}\left(\sum_{j=1}^{n-1} s_{j} \frac{\partial h}{\partial s_{j}}\left(s^{\prime}\right)-h\left(s^{\prime}\right)\right) \\
& \quad=(-1)^{n+1} \rho^{n-1}\left(\sum_{j=1}^{n} s_{j} \frac{\partial \lambda}{\partial \xi_{j}}(s)\right) / \frac{\partial \lambda}{\partial \xi_{n}}(s)=(-1)^{n+1} \rho^{n-1} / \frac{\partial \lambda}{\partial \xi_{n}}(s),
\end{aligned}
$$

because $\sum_{j=1}^{n-1} s_{j} \partial \lambda(s) / \partial \xi_{j}=\lambda(s)=1$ for $s \in S$. Thus we get $(6 \cdot 3)$ from the well known formula

$$
d S=\left(\sqrt{\sum_{i=1}^{n-1}}\left(\frac{\partial \bar{\lambda}}{\partial \xi_{j}}(s)\right)^{2}\left\|\frac{\partial \lambda}{\partial \xi_{n}}(s)\right\| d s^{\prime} .\right.
$$

Next we consider two functions $\lambda_{1}(\xi), \lambda_{2}(\xi)$ satisfying the conditions $(\lambda . \mathrm{i}),(\lambda$. ii) and the following.

(‥ iii) $S_{1}=\left\{s ; \lambda_{1}(s)=1\right\}$ and $S_{2}=\left\{s ; \lambda_{2}(s)=1\right\}$ have non empty intersection and $\operatorname{grad} \lambda_{1}(s) \neq \underset{\xi}{\operatorname{grad}} \lambda_{2}(s)$ for any $s \in S_{1} \cap S_{2}$. We set

$$
\begin{gathered}
\lambda(\hat{\xi}, \vartheta)=\vartheta \lambda_{1}(\xi)+(1-\vartheta) \lambda_{2}(\xi), \quad 0 \leq \vartheta \leq 1 . \\
S(\vartheta)=\{s ; \lambda(s, \vartheta)=1\} .
\end{gathered}
$$


Introducing in the same way the coordinate system $(\rho, s)$ :

$$
\xi=\rho s, 0<\rho<+\infty, s \in S(\vartheta) \text { for each } \vartheta \in[0,1],
$$

we have

$$
d \hat{\xi}=\left(\rho^{n-1} /|\underset{\xi}{\operatorname{grad}} \lambda(s, \vartheta)|\right) d \rho d S(\vartheta) .
$$

Let $\sigma^{\circ}$ be an arbitrarily fixed positive number and $\delta$ a positive number such that the closed interval $\left[\sigma^{\circ}-4 \delta, \sigma^{\circ}+4 \delta\right]$ does not contain 0 . Let $\psi \in C_{0}^{\infty}\left(\mathbb{R}^{1}\right)$ and satisfy $\operatorname{supp} \psi \subset\left\{\rho ;\left|\rho-\sigma^{\circ}\right|<4 \delta\right\}, \psi(\rho)=1$ for $\left|\rho-\sigma^{\circ}\right| \leq 3 \delta$. Then $\chi(\xi)=\chi(\rho s)=\psi(\rho), \quad 0<\rho<+\infty, \quad s \in S$ and $\chi_{\vartheta}(\xi)$ $=\chi_{\vartheta}(\rho s)=\psi(\rho), 0<\rho<+\infty, s \in S(\vartheta)$ define $C^{\infty}$ functions with compact support in $\Xi^{n} \backslash\{0\}$ such that $\chi(\xi)=1, \chi_{\vartheta}(\xi)=1$ in a neighborhood of $\sigma^{\circ} S$ and of $\sigma^{\circ} S(\vartheta)$ respectively. We consider the following functions.

$$
\begin{aligned}
\Phi(x ; \sigma \pm i \varepsilon)= & \int_{\Xi n} \frac{\exp \{i\langle x, \xi\rangle\} \chi(\xi) P(\xi)}{(\lambda(\xi)-(\sigma \pm i \varepsilon))^{j+1}} d \xi, \\
& \sigma \in\left[\sigma^{\circ}-\delta, \sigma^{\circ}+\delta\right], \varepsilon>0, \\
\Psi(x ; \sigma \pm i \varepsilon)= & \int_{\xi^{n}} \exp \{i\langle x, \xi\rangle\}\left(\int_{0}^{1} \frac{\chi_{\vartheta}(\xi) P(\xi, \vartheta)}{(\lambda(\xi, \vartheta)-(\sigma \pm i \varepsilon))^{2}} d \vartheta\right) d \xi, \\
& \sigma \in\left[\sigma^{\circ}-\delta, \sigma^{\circ}+\delta\right], \varepsilon>0 .
\end{aligned}
$$

Here $P(\xi)$ and $P(\xi, \vartheta)$ are positively homogeneous functions of degree $d \geq 0$ with respect to $\tilde{\xi}$ which are $C^{\infty}$ in $\mathbb{5}^{n} \backslash\{0\}$ and in $\left(\mathbb{g}^{n} \backslash\{0\}\right) \times[0,1]$ respectively. We shall study in this section the existence of the limits $\Phi(x ; \sigma \pm i 0)$ and the asymptotic behavior of $\Phi(x ; \sigma \pm i \varepsilon)$ as $|x| \rightarrow \infty$, and in the next section those for $\Psi(x ; \sigma \pm i \varepsilon)$. By shifting in (6.9) to the polar coordinates with respect to $S ;(\rho, s), 0<\rho<+\infty, s \in S$, we obtain

$$
\begin{aligned}
\Phi(x ; \sigma \pm i \varepsilon) & =\int_{0}^{\infty} \frac{\rho^{d+(n-1)} \psi(\rho)}{(\rho-(\sigma \pm i \varepsilon))^{j+1}} \\
& \times\left\{\int_{S} \exp \{i \rho\langle x, s\rangle\} \frac{P(s)}{\mid \operatorname{grad} \lambda(s) i} d S\right\} d \rho, \\
& \sigma \in\left[\sigma^{\circ}-\delta, \sigma^{\circ}+\delta\right], \quad \varepsilon>0 .
\end{aligned}
$$

Since $\phi(\rho)=1$ in $\left|\rho-\sigma^{\circ}\right| \leq 3 \delta$, the integrand in the integral with respect to $\rho$ can be extended to an analytic function of the complex variable $\rho$ in a domain including the interval $\left[\sigma^{\circ}-3 \delta, \sigma^{\circ}+3 \delta\right]$. Then we can deform 
the path $[0, \infty)$ in $(6 \cdot 11)$ so as to avoid the zeros of $\rho-(\sigma \pm i \varepsilon)$ for $\sigma \in\left[\sigma^{\circ}-\delta, \sigma^{\circ}+\delta\right], \varepsilon>0$. More precisely we replace the interval $\left[\sigma^{\circ}-2 \delta\right.$, $\left.\sigma^{\circ}+2 \delta\right]$ in the path $[0, \infty)$ in $(6 \cdot 11)$ by a semi-circle of radius $2 \delta$ and center at $\sigma^{\circ}$ in the lower half-plane or in the upper half-plane according to $\sigma+i \varepsilon$ or $\sigma-i \varepsilon$. Letting $\varepsilon$ tend to zero, we see that the limits $\Phi(x ; \sigma \pm i 0)$ exist and they define continuous functions of $(x, \sigma)$ in $\left(\boldsymbol{R}^{n} \backslash\{0\}\right) \times\left[\sigma^{\circ}-\delta, \sigma^{\circ}+\delta\right]$. In fact, they can be continued analytically across the real axis into a domain in the other half-plane.

In order to investigate the asymptotic behavior as $|x| \rightarrow \infty$ of $\Phi(x ; \sigma \pm i \varepsilon)$, we now assume the following:

( $\lambda$.iv) The Gaussian curvature $K(s)$ of $S$ never vanishes. Applying the formula $(5 \cdot 13)$ to the inner integral of $(6 \cdot 11)$, we find

$$
\begin{aligned}
& \Phi(x ; \sigma \pm i \varepsilon)=(2 \pi)^{(n-1) / 2}\left\{\frac{P(s(\theta)) \exp \left\{-\frac{i \pi}{4}(n-1)\right\}}{|K(s(\theta))|^{1 / 2} \mid \operatorname{grad} \lambda(s(\theta))} \mid\right. \\
& \times J_{s(\theta)}(x ; \sigma \pm i \varepsilon)+\frac{P(s(-\theta)) \exp \left\{\frac{i \pi}{4}(n-1)\right\}}{|K(s(-\theta))|^{1 / 2}|\operatorname{grad} \lambda(s(-\theta))|} \\
& \left.\quad \times J_{s(-\theta)}(x ; \sigma \pm i \varepsilon)\right\}|x|^{-(n-1) / 2} \\
& \quad+\int_{0}^{\infty} \frac{\rho^{(n-1,+d} \psi(\rho)}{(\rho-(\sigma \pm i \varepsilon))^{j+1}} q(\rho x) d \rho, \quad \sigma \in\left[\sigma^{\circ}-\delta, \sigma^{\circ}+\delta\right], \varepsilon \geq 0,
\end{aligned}
$$

where

$$
\begin{gathered}
J_{j, s}(x ; \sigma \pm i \varepsilon)=\int_{0}^{\infty} \frac{\rho^{(n-1) / 2+d} \psi(\rho)}{(\rho-(\sigma \pm i \varepsilon))^{j \mid 1}} \exp \{i \rho\langle x, s\rangle\} d \rho, \\
\sigma \in\left[\sigma^{\circ}-\delta, \sigma^{\circ}+\delta\right], \quad \varepsilon \geq 0 .
\end{gathered}
$$

Here we know that

$$
\begin{aligned}
\int_{-\infty}^{\infty} \frac{\exp \{i \rho l\}}{(\rho-(\sigma \pm i \varepsilon))^{j+1}} d \rho & = \pm 2 \pi i \frac{(i t)^{j}}{j !} Y( \pm t) \exp \{i(\sigma \pm i \varepsilon) t\} \\
j & =0,1, \cdots
\end{aligned}
$$

and

$$
\int_{-\omega}^{\nu} a(\rho) b(\rho) \exp \{i \rho t\} d \rho=(2 \pi)^{-1} \int_{-\omega}^{+\infty} a(t-\tau) b(\tau) d \tau
$$


where $Y(t)$ is the Heaviside function and

$$
\widetilde{a}(t)=\int_{-\infty}^{\infty} a(\rho) \exp \{i \rho t\} d \rho
$$

From $(6 \cdot 13),(6 \cdot 14),(6 \cdot 15)$ and $(6 \cdot 16)$, we get

$$
\begin{aligned}
J_{j, s}(x ; \sigma+i \varepsilon) & =\frac{i^{j+1}}{j !} \sum_{k=0}^{j}\left(\begin{array}{l}
j \\
k
\end{array}\right)\langle x, s\rangle^{k} \\
& \times \int_{-\infty}^{\langle x, s\rangle}(-\tau)^{j-k} \widetilde{\varphi}(\tau) \exp \{i(\sigma+i \varepsilon)(\langle x, s\rangle-\tau)\} d \tau,
\end{aligned}
$$

and

$$
\begin{aligned}
J_{j, s}(x ; \sigma-i \varepsilon) & =\frac{-i^{j+1}}{j !} \sum_{k=0}^{j}\left(\begin{array}{l}
j \\
k
\end{array}\right)\langle x, s\rangle^{k} \\
& \times \int_{\langle x, s\rangle}^{\infty}(-\tau)^{j-k} \tilde{\varphi}(\tau) \exp \{i(\sigma-i \varepsilon)(\langle x, s\rangle-\tau)\} d \tau,
\end{aligned}
$$

where $\varphi(\rho)=\rho^{(n-1) / 2+d} \phi(\rho)$. Thus the functions $J_{j, s}(x ; \sigma \pm i \varepsilon)$ are continuous in $\mathbb{R}^{n} \times \Delta_{ \pm}$respectively and

$$
J_{j, s}(x ; \sigma \pm i \varepsilon)=O\left(|x|^{j}\right) \quad \text { for } \quad|x| \rightarrow \infty, \sigma \pm i \varepsilon \in \Delta_{ \pm},
$$

where $\Delta_{ \pm}=\left\{\lambda=\sigma \pm i \varepsilon ;\left|\sigma-\sigma^{\circ}\right| \leq \delta, 0 \leq \varepsilon \leq \varepsilon^{\circ}\right\}$ and the order relation $O$ is uniform with respect to $\sigma \pm i \varepsilon \in A_{ \pm}$.

Since $S$ is a strictly convex closed hypersurface, we have

$$
\inf _{\theta \in \Omega}\langle\theta, s(\theta)\rangle>0 \text { and } \sup _{\theta \in \Omega}\langle\theta, s(-\theta)\rangle<0 .
$$

From these relations it follows that

$$
\begin{aligned}
& J_{j, s(-\theta)}(x ; \sigma+i \varepsilon)=O\left(|x|^{j-1}\right) \\
& \quad \text { and } J_{j, s(\theta)}(x ; \sigma-i \varepsilon)=O\left(|x|^{j-1}\right)
\end{aligned}
$$

for $|x| \rightarrow \infty, \theta=x /|x|$, where the order relation $O$ is uniform with respect to $(\theta, \sigma \pm i \varepsilon) \in \Omega \times \Delta_{ \pm}$. We also have

$$
\begin{aligned}
J_{j, s(\theta)}(x ; \sigma+i 0) & =\frac{i^{j+1}}{j !}\langle x, s(\theta)\rangle^{j} \\
& \times \int_{-\infty}^{\langle x, s(\theta)\rangle} \widetilde{\varphi}(\tau) \exp \{i \sigma(\langle x, s(\theta)\rangle-\tau)\} d \tau+O\left(|x|^{j-1}\right)
\end{aligned}
$$




$$
\begin{aligned}
= & \frac{i^{j+1}}{j !}\langle x, s(\theta)\rangle^{i} \exp \{i \sigma\langle x, s(\theta)\rangle\} \\
& \lambda \int_{-\infty}^{+\omega} \hat{\varphi}(\tau) \exp \{-i \sigma \tau\} d \tau+O\left(|x|^{j-1}\right) \\
= & \frac{2 \pi i}{j !}(i\langle x, s(\theta)\rangle)^{j} \sigma^{(n-1) / 2+d} \exp \{i \sigma|x|\langle\theta, s(\theta)\rangle\} \\
& +O\left(|x|^{j-1}\right)
\end{aligned}
$$

for $|x| \rightarrow \infty, \sigma \in\left[\sigma^{\circ}-\delta, \sigma^{\circ}+\delta\right]$ and

$$
\begin{gathered}
J_{j, s(-\theta)}(x ; \sigma-i 0)=\frac{-2 \pi i}{j !}(i\langle x, s(-\theta)\rangle)^{j} \sigma^{(n \quad j) / 2+d} \\
\quad \times \exp \{i \sigma|x|\langle\theta, s(-\theta)\rangle\}+O\left(|x|^{j-1}\right) \\
\quad \text { for } \quad|x| \rightarrow \infty, \sigma \in\left[\sigma^{\circ}-\bar{\delta}, \sigma^{\circ}+\grave{o}\right] .
\end{gathered}
$$

$(\partial / \partial x)^{\nu} \Phi_{j}(x ; \sigma \pm i \varepsilon)$ can be handled in the same way. 'Thus we obtain

$$
\begin{aligned}
\left(\frac{\partial}{\partial x}\right)^{\nu} & \Phi_{j}(x ; \sigma \pm i \varepsilon)=(2 \pi)^{(n-1) / 2} \frac{(i s( \pm \theta))^{\nu} P(s( \pm \theta))}{|K(s( \pm \theta))|^{1 / 2}|\operatorname{grad} \lambda(s( \pm \theta))|} \\
& \times \exp \{\mp i \pi(n-1) / 4\} I_{j, \nu, s( \pm \theta)}(x ; \sigma \pm i \varepsilon)|x|^{-(n-1) / 2+j} \\
& +O\left(|x|^{-(n+1) / 2+j}\right) \\
& +\int_{0}^{\infty} \frac{\rho^{d+|\nu|+n-1} \psi(\rho)}{(\rho-(\sigma \pm i \varepsilon))^{j+1}} q(\rho x) d \rho, \text { as }|x| \rightarrow \infty, \theta=x /|x|
\end{aligned}
$$

where

$$
I_{j, \nu, s}(x ; \sigma \pm i \varepsilon)=|x|^{-j} \int_{0}^{\omega} \frac{\rho^{(n-1) / 2+d+|\nu|} \psi(\rho)}{(\rho-(\sigma \pm i \varepsilon))^{j+1}}-\exp \{i \rho\langle x, s\rangle\} d \rho
$$

and the functions $I_{j, \nu, s( \pm \theta)}(x ; \sigma \pm i \varepsilon)$ are bounded continuous in $\{x$; $|x| \geq 1\} \times \Delta_{ \pm}$.

To estimate the last integral in the second member of $(6 \cdot 24)$, we give here two lemmas.

Lemma 6. 1. $1^{\circ}$ Let $I=(\alpha, \beta)$ be a bounded open interval in $\mathrm{T}=(-\infty, \infty)$ and $f(\rho)$ a Höldcr continuous function of order $\vartheta(0<\vartheta \leq 1)$ defined in $I$, that is

$$
\| f \mathbb{V}_{\vartheta}=\sup _{\rho \in I}|f(\rho)|+\sup _{\substack{\rho, \rho^{\prime} \in I \\ \rho \neq \rho^{\prime}}}\left\{\left|f(\rho)-f\left(\rho^{\prime}\right)\right| /\left|\rho-\rho^{\prime}\right|^{\vartheta}\right\}<\infty .
$$


Then the integral

$$
F(\sigma \pm i \varepsilon ; f)=\int_{I} \frac{f(\rho)}{\rho-(\sigma \pm i \varepsilon)} d \rho
$$

exists for every $\sigma \in I$ and every $\varepsilon \geq 0$ and there exists, for any compact

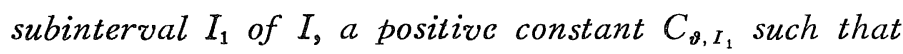

$$
|F(\sigma \pm i \varepsilon ; f)| \leq C_{\vartheta, I_{1}} \mid f \|_{\vartheta} \text { for } \sigma \in I_{1} \text { and } \varepsilon \geq 0
$$

where $C_{\vartheta, I_{1}}$ dcpends on $\vartheta$ and $I_{1}$ but not on $\varepsilon \geq 0$ and $f$.

$2^{\circ}$ Further, if $0<\vartheta<1$, the function $F(\sigma \pm i \varepsilon ; f)$ is Hölder continuous of order $\vartheta$ and there exists a positive constant $C_{\vartheta, I_{1}}^{\prime}$ such that $(6 \cdot 29)\left|F(\sigma \pm i \varepsilon ; f)-F\left(\sigma^{\prime} \pm i \varepsilon ; f\right)\right| \leq C_{\vartheta, I_{1}}^{\prime}\left|\sigma-\sigma^{\prime}\right|^{\vartheta}|f|_{\vartheta} \quad$ for $\quad \sigma, \sigma^{\prime} \in I_{1}$ and $\varepsilon \geq 0$, where $C_{\vartheta, I_{1}}^{\prime}$ depends on $\vartheta$ and $I_{1}$ but not on $\varepsilon \geq 0$ and $f$. If $\vartheta=1$, (6.29) holds for every $\vartheta \in(0,1)$.

Remark. The second statement is usually called "lemma of Privalov" (see Friedrichs [4]).

Proof. $1^{\circ}$ We will only discuss the case $\sigma+i \varepsilon$, for the other case can be handled in the same way. Define $f(x)=0$ outside of $I$. Then we have

$$
\begin{aligned}
F(\sigma+i \varepsilon ; f) & =\int_{-N}^{N} \frac{f(\rho+\sigma)}{\rho-i \varepsilon} d \rho \text { for } \sigma \in I \text { and } \quad \hat{<}>0 \\
& =\int_{-N}^{N} \frac{f(\rho+\sigma)-f(\sigma)}{\rho-i \varepsilon} d \rho+f(\sigma) \int_{-N}^{N} \frac{1}{\rho-i \varepsilon} d \rho \\
& =\int_{-N}^{N} \frac{f(\rho+\sigma)-f(\sigma)}{\rho-i \varepsilon} d \rho+f(\sigma)\left(\log \frac{N-i \varepsilon}{N+i \varepsilon}+i \pi\right)
\end{aligned}
$$

where $N$ is a suitable positive number and $\log z$ denotes the principal value of $\log z$. From the Hölder continuity in $I$ of $f$, we see that the limit

$$
F(\sigma+i 0 ; f)=\lim _{\varepsilon \rightarrow 0+} F(\sigma+i \varepsilon ; f)=\int_{-N}^{N} \frac{f(\rho+\sigma)-f(\sigma)}{\rho} d \rho+i \pi f(\sigma)
$$

exists for every $\sigma \in I$ and 


$$
F(\sigma+i 0 ; f)=\text { p.v. } \int_{-\infty}^{\omega} \frac{f(\rho)}{\rho-\sigma} d \rho+i \pi f(\sigma)
$$

Here the symbol p.v. means the Cauchy principal value. Thus for $\varepsilon \geq 0$ we have

$$
\begin{aligned}
|F(\sigma+i \varepsilon ; f)| & \leq \int_{-N}^{N}\left|\frac{f(\rho+\sigma)-f(\sigma)}{\rho-i \varepsilon}\right| d \rho+\left|i \pi+\log \frac{N-i \varepsilon}{N+i \varepsilon}\right||f(\sigma)| \\
& \leq \int_{-N}^{N}\left|\frac{f(\rho+\sigma)-f(\sigma)}{\rho}\right| d \rho+\pi \sup _{\rho \in I}|f(\rho)| .
\end{aligned}
$$

Choose $\hat{0}>0$ so that $I_{1} \subset(\alpha+\delta, \beta-\delta)$ and let

$$
\begin{aligned}
& \int_{-N}^{N}\left|\frac{f(\rho+\sigma)-f(\sigma)}{\rho}\right| d \rho=\int_{-\grave{o}}^{\grave{o}} "+\int_{-N}^{-\grave{o}} " 1+\int_{\delta}^{N} " \\
& \equiv K_{1}+K_{2}+K_{3} \text {, respectively. }
\end{aligned}
$$

Now

$$
\left|K_{1}\right| \leq|f|_{\vartheta} \int_{-\hat{o}}^{\grave{o}}|\rho|^{\vartheta-1} d \rho=2 \hat{o}|f|_{\vartheta} / \vartheta
$$

On the other hand

$$
\left|K_{2}\right|,\left|K_{3}\right| \leq 2 \sup _{\rho \in I}|f(\rho)| \cdot \log (N / \tilde{0}) .
$$

Hence $(6 \cdot 28)$ holds with $C_{\vartheta, I_{1}}=2 \delta / \vartheta+2 \log (N / 0)$.

$2^{\circ}$ Next we proceed to show the second statement. For $\sigma, \sigma^{\prime} \in I_{1}$, $\sigma^{\prime}<\sigma$, we set

$$
\sigma-\sigma^{\prime}=2 b(>0), \quad \sigma+\sigma^{\prime}=2 \tau .
$$

'Then we have

$$
\begin{aligned}
& F(\sigma+i \varepsilon ; f)-F\left(\sigma^{\prime}+i \varepsilon ; f\right)=\left(\sigma-\sigma^{\prime}\right) \int_{-\omega}^{\omega} \overline{(\rho-\sigma-i \varepsilon)\left(\rho-\sigma^{\prime}-i \varepsilon\right)} d \rho \\
& =2 b \int_{-\infty}^{\tau} \frac{f(\rho)}{(\rho-\sigma-i \varepsilon)\left(\rho-\sigma^{\prime}-i \varepsilon\right)} d \rho+2 b \int_{\tau}^{\infty} \frac{f(\rho)}{(\rho-\sigma-i \varepsilon)\left(\rho-\sigma^{\prime}-i \varepsilon\right)} d \rho \\
& =2 b \int_{-\infty}^{b} \frac{f\left(\rho+\sigma^{\prime}\right)}{(\rho-2 b-i \varepsilon)(\rho-i \varepsilon)} d \rho+2 b \int_{-b}^{\omega} \frac{f(\rho+\sigma)}{(\rho-i \varepsilon)(\rho+2 b-i \varepsilon)} d \rho \\
& =2 b \int_{-\infty}^{b} \frac{f\left(\rho+\sigma^{\prime}\right)-f\left(\sigma^{\prime}\right)}{(\rho-2 b-i \varepsilon)(\rho-i \varepsilon)} d \rho+2 b f\left(\sigma^{\prime}\right) \int_{-\infty}^{o} \frac{1}{(\rho-2 b-i \varepsilon)(\rho-i \varepsilon)} d \rho \\
& \quad+2 b \int_{-b}^{\infty} \frac{f(\rho+\sigma)-f(\sigma)}{(\rho-i \varepsilon)(\rho+2 b-i \varepsilon)} d \rho+2 b f(\sigma) \int_{-b}^{\infty} \frac{1}{(\rho-i \varepsilon)(\rho+2 b-i \varepsilon)} d \rho .
\end{aligned}
$$


Now

$$
2 b \int_{-\infty}^{b} \frac{1}{(\rho-2 b-i \varepsilon)(\rho-i \varepsilon)} d \rho==\log \frac{b+i \varepsilon}{b-i \varepsilon}-i \pi
$$

and

$$
2 b \int_{-b}^{\infty} \frac{1}{(\rho-i \varepsilon)(\rho+2 b-i \varepsilon)} d \rho=i \pi-\log \frac{b+i \varepsilon}{b-i \varepsilon} .
$$

Therefore for $\varepsilon>0$ we find

$$
\begin{aligned}
F(\sigma+ & i \varepsilon ; f)-F\left(\sigma^{\prime}+i \varepsilon ; f\right)=\left(i \pi-\log \frac{b+i \varepsilon}{b-i \varepsilon}\right)\left(f(\sigma)-f\left(\sigma^{\prime}\right)\right) \\
& +\left(\sigma-\sigma^{\prime}\right) \int_{-\infty}^{b} \frac{f\left(\rho+\sigma^{\prime}\right)-f\left(\sigma^{\prime}\right)}{(\rho-i \varepsilon)(\rho-2 b-i \varepsilon)} d \rho \\
& +\left(\sigma-\sigma^{\prime}\right) \int_{b}^{\infty} \frac{f(\rho+\sigma)-f(\sigma)}{(\rho-i \varepsilon)(\rho+2 b-i \varepsilon)} d \rho
\end{aligned}
$$

In this formula we can let $\varepsilon$ tend to zero on account of the Hölder continuity in $I$ of $f$. So we have for $\varepsilon \geq 0$

$$
\begin{aligned}
& \frac{\left|F(\sigma+i \varepsilon ; f)-F\left(\sigma^{\prime}+i \varepsilon ; f\right)\right|}{\left|\sigma-\sigma^{\prime}\right|^{\vartheta}} \leq \pi \frac{\left|f(\sigma)-f\left(\sigma^{\prime}\right)\right|}{\left|\sigma-\sigma^{\prime}\right|^{\vartheta}} \\
& \quad+(2 b)^{1-\vartheta} \int_{-\infty}^{b} \frac{\left|f\left(\rho+\sigma^{\prime}\right)-f\left(\sigma^{\prime}\right)\right|}{|(\rho-i \varepsilon)(\rho-2 b-i \varepsilon)|} d \rho \\
& \quad+(2 b)^{1-\vartheta} \int_{-b}^{\infty} \frac{|f(\rho+\sigma)-f(\sigma)|}{|(\rho-i \varepsilon)(\rho+2 b-i \varepsilon)|} d \rho \\
& \equiv J_{1}+J_{2}+J_{3}, \text { respectively. }
\end{aligned}
$$

Consider $J_{2}$ :

$$
\begin{aligned}
\left|J_{2}\right| & \leq 2 b^{1-\vartheta} \int_{-\infty}^{b} \frac{\left|f\left(\rho+\sigma^{\prime}\right)-f\left(\sigma^{\prime}\right)\right|}{|\rho(\rho-2 b)|} d \rho \\
& \leq 2|f|_{\vartheta} b^{1-\vartheta} \int_{-\delta}^{b} \frac{1}{|\rho|^{1-\vartheta}|\rho-2 b|} d \rho+2 b \cdot \sup _{\rho \in I}|f(\rho)| \int_{-\infty}^{-\delta} \frac{1}{|\rho(\rho-2 b)|} d \rho .
\end{aligned}
$$

Now

$$
\begin{gathered}
b^{1-\vartheta} \int_{-\delta}^{b} \frac{1}{|\rho|^{1-\vartheta}|\rho-2 b|} d \rho \leq \int_{-\infty}^{1} \frac{1}{|t|^{1-\vartheta}|t-2|} d t \\
\leq 2 \int_{0}^{1} t^{\vartheta-1} d t+\int_{1}^{\infty} t^{\vartheta-2} d t \leq 2 / \vartheta(1-\vartheta)
\end{gathered}
$$


and

$$
2 b \int_{-\infty}^{-\grave{o}} \mid \frac{1}{|\rho(\rho-2 b)|} d \rho=2 b \int_{\hat{\delta}}^{\infty} \frac{1}{\rho(\rho+2 b)} d \rho=\log \frac{\hat{o}+2 b}{\delta} .
$$

Hence

$$
\left|J_{2}\right| \leq\left(\frac{4}{\vartheta(1-\vartheta)}+\log \frac{\delta+2 b}{\delta}\right)|f|_{\diamond}
$$

In the same way

$$
\left|J_{3}\right| \leq\left(\frac{4}{\vartheta(1-\vartheta)}+\log \frac{\partial+2 b}{\delta}\right)|f|_{\vartheta}
$$

From $(6 \cdot 33),(6 \cdot 34)$ and $(6 \cdot 35)$ we have $(6 \cdot 29)$ with $C_{\vartheta, J_{1}}=\pi+\frac{8}{\vartheta(1-\vartheta)}$ $+2 \log \frac{\partial+2 b}{\delta}$. This completes the proof.

Lemma 6. 2. Let $q(x)$ be a $C^{\omega}$ function in $\mathbb{R}^{n}$ such that for cvery multi-index $\nu$ with $|\nu| \leq j+1$ ( $j$ is a fixed integer $\geq 0$ ) the estimate

$$
\left|(\partial / \partial x)^{\nu} q(x)\right| \leq C_{j}|x|^{-(n+1) / 2} \text { for }|x| \geq R_{j}
$$

is valid for some constants $C_{j}$ and $R_{j}$. Let $I_{1}$ be a closed interval in $\mathbf{R}_{+}=(0, \infty)$ and let $\omega(\rho)$ be a $C^{\circ}$ Sunction roith compact support in $\mathbf{R}_{+}$which is 1 in a neighborhood of $I_{1}$. Then for every $\gamma$ with $0<\gamma<1$ we have

$$
\left|\int_{0}^{\infty} \frac{\omega(\rho) q(\rho x)}{(\rho-(\sigma \pm i \varepsilon))^{j+1}} d \rho\right| \leq C_{I_{1}, j}|x|^{-(n-1) / 2-\gamma+j}
$$

for $|x| \sum M_{I_{1}, j}$ and $\sigma \in I_{1}$. Here $C_{I_{1, j}, j}$ and $M_{I_{1}, j}$ are positive constants and $C_{I_{1}, r, j}$ is independent of $\sigma \in I_{1}$ and $\varepsilon \geq 0$ and $M_{I_{1}, j}$ independent of $\sigma \in I_{1}, r \in(0,1)$ and $\varepsilon \geq 0$.

Proof. It is sufficient to show (6.37) for $j=0$, since we can reduce the general case to this case by integration by parts. Take a bounded open interval $I=(\alpha, \beta)$ such that $\omega(\rho)=1$ on $I$ and set

$$
f_{x}(\rho)=\omega(\rho) q(\rho x)
$$

From the assumption $(6 \cdot 36)$ there exists a constant $C_{I}$ clepending on $I$ such that 


$$
\left|f_{x}(\rho)\right| \leq C_{I}|x|^{-(n+1) / 2} \quad \text { for } \quad|x| \geq R_{\circ} / \alpha \text {. }
$$

Consider for $\rho, \rho^{\prime} \in I$

$$
\frac{\left|q(\rho x)-q\left(\rho^{\prime} x\right)\right|}{\left|\rho-\rho^{\prime}\right|^{1-r}}=\left|\frac{q(\rho x)-q\left(\rho^{\prime} x\right)}{\rho-\rho^{\prime}}\right|^{1-r}\left|q(\rho x)-q\left(\rho^{\prime} x\right)\right|^{r} .
$$

From $(6 \cdot 36)$ we have

$$
\left|q(\rho x)-q\left(\rho^{\prime} x\right)\right|^{r} \leq C_{r, I}|x|^{-(n+1) r / 2} \text { for } \quad|x| \geq R_{\circ} / \alpha
$$

and

$$
\begin{gathered}
\left|\frac{q(\rho x)-q\left(\rho^{\prime} x\right)}{\rho-\rho^{\prime}}\right|^{1-1}=\left|\sum_{j=1}^{n} x_{j} \frac{\partial q}{\partial x_{j}}\left(\left(\rho^{\prime}+\left(\rho-\rho^{\prime}\right) \theta\right) x\right)\right|^{1-r} \\
\leq C_{r, I}|x|^{(-n+1)(1-r) / 2} \text { for } \quad\left|x_{\mid}\right| \geq R_{\circ} / \alpha .
\end{gathered}
$$

Hence

$(6 \cdot 40)$

$$
\begin{gathered}
\left|q(\rho x)-q\left(\rho^{\prime} x\right)\right| /\left|\rho-\rho^{\prime}\right|^{1-r} \leq C_{r, I}|x|^{-(n-1) / 2-r} \\
\text { for }|x| \geq R_{\circ} / \alpha .
\end{gathered}
$$

From $(6 \cdot 39)$ and $(6 \cdot 40)$ it follows that

$$
\begin{gathered}
\left|f_{x}(\rho)-f_{x}\left(\rho^{\prime}\right)\right| /\left|\rho-\rho^{\prime}\right|^{1-r} \leq C_{r, I}|x|^{-(n-1) / 2-r} \\
\text { for }|x| \geq R_{\circ} / \alpha .
\end{gathered}
$$

Thus we obtain

$$
\left|f_{x}(\rho)\right|_{1-\gamma} \leq \text { const. }{ }_{r, I}|x|^{-(n-1) / 2-\gamma} \text { for }|x| \geq R_{\circ} / \alpha .
$$

Applying $1^{\circ}$ of Lemma 6.1 to $f_{x}(\rho)$ we have (6.37) for $j=0$ from $(6 \cdot 41)$. Therefore the proof is complete.

Summing up the preceding arguments in this section we have proved

Theorem 6.3. Let $\Phi_{j}(x ; \sigma \pm i \varepsilon)$ be the function defined by (6.9). Then $(\partial / \partial x)^{\nu} \Phi_{j}\left(x ; \sigma \pm i_{\varepsilon}\right)$ has the following asymptolic formula for $|x| \rightarrow \infty$.

$$
\begin{aligned}
& (\partial / \partial x)^{\nu} \Phi_{j}(x ; \sigma \pm i \varepsilon)=(2 \pi)^{(n-1) / 2} \frac{(i s( \pm \theta))^{\nu} P(s( \pm \theta))}{|K(s( \pm \theta))|^{1 / 2} \mid \operatorname{grad} \lambda(s( \pm \theta)) !} \\
& \quad \times \exp \{\mp i \pi(n-1) / 4\} I_{j, \nu, s( \pm \theta)}(x ; \sigma \pm i \varepsilon)|x|^{-(n-1) / 2+j} \\
& \quad+O\left(|x|^{-(n-1) / 2-\gamma+j}\right)
\end{aligned}
$$




$$
\text { for }|x| \rightarrow \infty, \theta=x /|x|, \sigma \in\left[\sigma^{\circ}-\delta, \sigma^{\circ}+\delta\right], \varepsilon \geq 0 \text {. }
$$

Here

$$
I_{j, \nu, s}(x ; \sigma \pm i \varepsilon)=|x|^{-j} \int_{0}^{\infty} \frac{\rho^{(n-1) / 2+d+|\nu|} \psi(\rho)}{(\rho-(\sigma \pm i \varepsilon))^{j+1}} \exp \{i \rho\langle x, s\rangle\} d \rho
$$

and the functions $I_{j, \nu, s( \pm 0)}(x ; \sigma \pm i \varepsilon)$ are bounded continuous in $\{x ;|x|$ $\geq 1\} \times \Delta_{ \pm}$. Further the order relation $O$ in (6.42) is uniform with respect to $(\theta, \sigma \pm i \varepsilon) \in \Omega \times \Delta_{ \pm}$. In particular

$$
\begin{aligned}
& (\partial / \partial x)^{\nu} \Phi_{j}(x ; \sigma \pm i 0)= \pm(2 \pi)^{(n+1) / 2} i^{|\nu|+i+1} \\
& \times \frac{(s( \pm \theta))^{\nu}\langle 0, s( \pm \theta)\rangle^{j} P(s( \pm \theta))}{j !|K(s( \pm \theta))|^{1 / 2}|\operatorname{grad} \lambda(s( \pm \theta))|} \sigma^{(n-1) / 2+d+|\nu|} \\
& \times \exp \{\mp i \pi(n-1) / 4+i \sigma\langle x, s( \pm \theta)\rangle\}|x|^{-(n-1, / 2+i} \\
& +O\left(|x|^{-(n-1) / 2-r+i}\right) \\
& \quad \text { for } \quad|x| \rightarrow \infty, 0=x /|x|, \sigma \in\left[\sigma^{\circ}-\hat{o}, \sigma^{\circ}+\delta\right] .
\end{aligned}
$$

Moreover we obtain for following estimate making use of $2^{\circ}$ of Lemma 6. 1.

$$
\begin{aligned}
& \left|(\partial / \partial x)^{\nu}\left\{\Phi_{i}(x ; \sigma \pm i \varepsilon)-\bar{\Phi}_{j}\left(x ; \sigma^{\prime} \pm i \varepsilon\right)\right\}\right| \\
& \quad \leq C_{i, \nu, \gamma, \hat{o}}\left|\sigma-\sigma^{\prime}\right| \gamma|x|^{-(n-3) / 2+i} \\
& \quad \text { for } \quad|x| \geq R_{i, \hat{r}}, \sigma, \sigma^{\prime} \in\left[\sigma^{\circ}-\hat{o}, \sigma^{\circ}+\hat{0}\right], \quad \therefore \geq 0 .
\end{aligned}
$$

\section{$\S 7$. Basic Formulas II}

In this section we shall investigate the asymptotic behavior of the function $\Psi(x ; \sigma \pm i \varepsilon)$ defined by $(6 \cdot 10)$. By transforming to the polar coordinates with respect to $S(\vartheta):(\rho, s), 0<\rho<+\infty, s \in S(\vartheta)$, we find

$$
\begin{gathered}
\Psi(x ; \sigma \pm i \varepsilon)=\int_{0}^{1} d \vartheta \int_{\Xi^{n}} \frac{\chi_{\vartheta}(\xi) P(\xi, \vartheta)}{(\lambda(\xi, \vartheta)-(\sigma \pm i \varepsilon))^{2}} \exp \{i\langle x, \xi\rangle\} d \xi \\
=\int_{0}^{\infty} \frac{\rho^{n-i+d} \psi(\rho)}{(\rho-(\sigma \pm i \varepsilon))^{2}}\left\{\int_{0}^{1} d \vartheta \int_{S(\vartheta)} \frac{P(s, \vartheta)}{|\operatorname{grad} \lambda(s, \vartheta)|}\right. \\
\quad \times \exp \{i \rho\langle x, s\rangle\} d S(\vartheta)\} d \rho .
\end{gathered}
$$

The existence of the limits $\Psi(x ; \sigma \pm i 0)$ can be proved by the same 
argument as in the case of the function $\Phi(x ; \sigma \pm i \varepsilon)$ defined by (6.9). In order to derive the asymptotic formula for $\Psi(x ; \sigma \pm i \varepsilon)$ as $|x| \rightarrow \infty$, we must study the asymptotic behavior as $|x| \rightarrow \infty$ of the integral of the form

$$
I(x)=\int_{0}^{1} d \vartheta \int_{S(\vartheta)} \mu(s, \vartheta) \exp \{i\langle x, s\rangle\} d S(\vartheta)
$$

where $\mu(s, \vartheta)$ is a $C^{\infty}$ function on $S(\vartheta)$ which depends infinitely differentiably on $\vartheta \in[0,1]$. For this purpose we first start the following theorem required presently.

Theorem \%.1. If the Gaussian curvatures of $S_{1}=\left\{s ; \lambda_{1}(s)=1\right\}$ and $S_{2}=\left\{s ; \lambda_{2}(s)=1\right\}$ never vanish on $S_{1}$ and $S_{2}$ respectively, the Gaussian curvature $K(s, \vartheta)$ of $S(\vartheta)$ never vanishes on $S(\vartheta)$ for any $\vartheta \in(0,1)$.

For the proof we need an elementary lemma.

Lemma 7.2. Let $E$ be a symmetric matrix with real entries of the form

$$
E=\left(\begin{array}{ll}
E_{\circ} & \mathfrak{a} \\
\iota_{\mathfrak{a}} & \mathfrak{b}
\end{array}\right),
$$

where $\mathfrak{a}$ is an $(n-1) \times 1$ matrix, $\mathfrak{b}$ a real number and ${ }^{t} \mathfrak{a}$ denotes the transposed matrix of $\pi$. If the $(n-1) \times(n-1)$ matrix $E_{\circ}$ is positive definite and $E$ has zero as an eigenvalue, then $E$ is nonnegative definite and its kernel ker $E$ has the dimension 1. Conversely, if $E$ is nonnegative definite and $\operatorname{ker} E=\{\alpha \zeta ; \alpha \in \mathbb{R}\}$ for some fixed real vector $\zeta \in \mathbb{\Xi}^{n}$ with $\zeta_{n} \neq 0$, then $E_{\circ}$ is positive definite.

Proof. Let us set

$$
T^{Y}=\left(\begin{array}{cc}
I_{n-1} & E_{\circ}^{-1} \mathfrak{a} \\
0 \cdots 0 & 1
\end{array}\right),
$$

where $I_{n-1}$ is the identity matrix of order $n-1$. Then we have 


$$
E={ }^{t} V^{\gamma}\left(\begin{array}{cc}
E_{0} & 0 \\
& \vdots \\
0 \cdots 0 & b-\left\langle E_{o}{ }^{-1} \mathfrak{a}, a\right\rangle
\end{array}\right) T^{\top}
$$

and det $I^{r}=1$. Therefore

$$
\operatorname{det} E=\left(\operatorname{det} E_{\circ}\right)\left(\mathfrak{b}-\left\langle E_{\circ}{ }^{-1} \mathfrak{a}, \mathfrak{a}\right\rangle\right) .
$$

Since $\operatorname{det} E_{0} \neq 0$, we have $\mathfrak{b}=\left\langle E_{\circ}{ }^{-1} \mathfrak{a}, \mathfrak{a}\right\rangle$. Consider the transformation in $\boldsymbol{\Xi}^{n}: \xi=T^{\top} \eta$. Then

$$
\langle E \xi, \xi\rangle=\left\langle\left.{ }^{t}\right|^{r} E V^{\top} \eta, \eta\right\rangle=\left\langle\left(\begin{array}{cc}
E_{\circ} & 0 \\
0 & 0
\end{array}\right) \eta, \eta\right\rangle \geq 0 .
$$

Thus $E$ is nonnegative definite. Since $V$ is regular, $\operatorname{dim} \operatorname{ker} E=\operatorname{dim}$ ker $\left(\begin{array}{ll}E_{\circ} & 0 \\ 0 & 0\end{array}\right)$. Hence ker $E$ has the dimension 1.

Conversely, if $E$ is nonnegative definite, we have

$$
\langle E \xi, \xi\rangle=\left\langle E_{\circ} \xi^{\prime}, \xi^{\prime}\right\rangle \geq 0 \text { for any } \xi=\left(\begin{array}{c}
\xi^{\prime} \\
0
\end{array}\right) \in \mathbb{\Xi}^{n} \text {. }
$$

Suppose that there exists a vector $\xi^{\prime \prime} \in \mathbb{E}^{n-1} \backslash\{0\}$ such that $E_{0} \xi^{\prime}=0$. Then

$$
0=\langle E \xi, \xi\rangle=\left\langle E^{1 / 2} \xi, E^{1 / 2} \xi\right\rangle .
$$

Therefore

$$
E^{1 / 2} \xi=0
$$

Since $\operatorname{ker} E=\operatorname{ker} E^{1 / 2}$, we have $\xi \in \operatorname{ker} E$. This contradicts that ker $E$ $=\{\alpha \zeta ; \alpha \in \mathbf{R}\}$ for some $\zeta \in \mathbb{G}^{n}$ with $\zeta_{n} \neq 0$. Thus $E_{\circ}$ must be positive definite.

Proof of Theorem 7.1. Let $u(\xi)$ be a real valued function of $\xi \in \mathbb{G}^{n}$ which is positively homogeneous of degree 1 , infinitely differentiable and $u(\xi)>0$ for $\xi \neq 0$. Let $s^{\circ}$ be an arbitrarily fixed point of the hypersurface $\Sigma=\{s ; u(s)=1\}$. Then there exists a suitable orthogonal transformation $\xi=T \eta$ such that

$\left.(7 \cdot 4) \quad \frac{\partial u}{\partial \eta_{j}}\left(T_{\eta}\right)\right|_{\eta=\sigma^{\circ}}=0, j=1, \cdots, n-1,\left.\frac{\partial u}{\partial \eta_{n}}\left(T_{\eta}\right)\right|_{\eta=\sigma^{\circ}}>0, s^{\circ}=T \sigma^{\circ}$. 
In fact it suffices to choose the transformation in such a way that the direction of the outward normal at $s^{\circ}$ to the surface $\Sigma$ coincides with the positive direction of $\eta_{n}$ axis in $\eta$ coordinate system. The surface $\Sigma$ can be represented by the equation of the form $\eta_{n}=h\left(\eta_{1}, \cdots, \eta_{n-1}\right)$ is a neighborhood of $\sigma^{\circ}$ and we have

$$
\left(-\frac{\partial^{2} h}{\partial \eta_{j} \partial \eta_{k}}\left(\sigma^{\circ}\right)\right)_{j, k=1, \cdots, n-1}=\left(\frac{\frac{\partial^{2} u}{\partial \eta_{j} \partial \eta_{k}}\left(T \sigma^{\circ}\right)}{\frac{\partial u}{\partial \eta_{n}}\left(T \sigma^{\circ}\right)}\right)_{j, k=1, \cdots, n-1} .
$$

The determinant of the matrix of the left-hand side equals the Gaussian curvature of $\Sigma$ at the point $s^{\circ}$ ( $\sigma^{\circ}$ in $\eta$ coordinate system). If the Gaussian curvature of $\Sigma$ never vanishes on $\Sigma$, the matrix of the lefthand side of $(7 \cdot 5)$ is positive definite and therefore the matrix $\left(\partial^{2} u\left(T \sigma^{\circ}\right) /\right.$ $\left.\partial \eta_{j} \partial \eta_{k}\right)_{j, k=1, \ldots, n-1}$ is so. Conversely, if the matrix $\left(\partial^{2} u\left(T \sigma^{\circ}\right) / \partial \eta_{j} \partial \eta_{k}\right)_{j, k=1, \ldots}$, ${ }_{n-1}$ is positive definite, the Gaussian curvature of $\Sigma$ at the point $s^{\circ}$ does not vanish. Now we note the following.

The Hessian matrix of $u(\xi)$ at $\xi \neq 0$ has zero as an eigenvalue and $\xi$ is a corresponding eigenvector.

Indeed, this follows from the positive homogeneity of degree 1 of $u(\xi)$ and from Euler's identity:

$$
\left(\frac{\partial^{2} u}{\partial \xi_{j} \partial \xi_{k}}(\xi)\right)_{j, k=1, \ldots, n}\left(\begin{array}{c}
\xi_{1} \\
\vdots \\
\xi_{n}
\end{array}\right)=\left(\sum_{k=1}^{n} \frac{\partial^{2} u}{\partial \xi_{j} \partial \xi_{k}}(\xi) \xi_{k}\right)_{j=1, \ldots, n}=0 .
$$

Now let $s^{\circ}$ be an arbitrarily fixed point of the surface $S(\vartheta), \vartheta$ being fixed. For $u(\xi)=\lambda(\xi, \vartheta)$, choose an orthogonal transformation $\xi=T(\vartheta) \eta$ such that

$$
\frac{\partial \lambda}{\partial \eta_{j}}\left(T(\vartheta) \sigma^{\circ}, \vartheta\right)=0, j=1, \cdots, n-1, \frac{\partial \lambda}{\partial \eta_{n}}\left(T(\vartheta) \sigma^{\circ}, \vartheta\right)>0,
$$

where $\lambda(\xi, \vartheta)=\vartheta \lambda_{1}(\xi)+(1-\vartheta) \lambda_{2}(\xi)$ and $s^{\circ}=T(\vartheta) \sigma^{\circ}$. On the other hand there exist positive numbers $\rho_{\iota}>0$ and $s^{\imath} \in S_{\iota}$ such that $\rho_{\iota} s^{\imath}=s^{\circ}$, $\iota=1,2$. We choose orthogonal transformations $\xi=T_{\iota} \eta^{\iota}, \iota=1,2$ such that

$$
\frac{\partial \lambda_{c}}{\partial \eta_{j}{ }^{c}}\left(T_{\iota} \sigma^{\iota}\right)=0, j=1, \cdots, n-1, \frac{\partial \lambda_{c}}{\partial \eta_{j}{ }^{c}}\left(T_{\iota} \sigma^{\iota}\right)>0, \iota=1,2,
$$


where $s^{\iota}=T_{\iota} \sigma^{\imath}, \iota=1,2$. By the assumplion on Gaussian curvatures of $S_{\iota}, \iota=1,2$ and the corresponding relations to $(7 \cdot 5)$, the matrices $\left(\partial^{2} \lambda_{\iota}\left(T_{\iota} \sigma^{\iota}\right) / \partial \eta_{l}^{\iota} \partial \eta_{j}^{\iota}\right)_{j, k-1, \cdots, n-1}(\iota=1,2)$ of order $n-1$ are positive definite. Consequently, by the first statement of Lemma 7.2, the Hessian matrices $\left(\partial^{2} \lambda_{\iota}\left(T_{\iota} \sigma^{\iota}\right) / \partial \eta_{j}^{\iota} \partial \eta_{k}^{\iota}\right)_{j, k=1, \ldots, n}$ of $\lambda_{\iota}\left(T_{\iota} \eta^{\iota}\right)$ (relative to $\eta^{\iota}$ ) at $\eta^{\iota}=\sigma^{\iota}$ are nonnegative definite and have zero as an eigenvalue. Now we have

$$
\begin{aligned}
& \left(\frac{\partial^{2} \lambda_{l}}{\partial \eta_{j} \partial \eta_{k}}\left(T(\vartheta) \sigma^{\circ}\right)\right)_{j, k=1, \ldots, n}={ }^{t}\left({ }^{t} T, T(\vartheta)\right)\left(\frac{\partial^{2} \lambda_{l}}{\partial \eta_{j}^{l} \partial \eta_{l}^{\iota}}\left(s^{\circ}\right)\right)_{j, k=1, \ldots, n}\left({ }^{l} T_{\iota} T(\vartheta)\right) \\
& =\rho_{\iota}{ }^{-n t}{ }^{t}\left({ }^{t} T, T(\vartheta)\right)\left(\frac{\partial^{2} \lambda_{\iota}}{\partial \eta_{j}^{c} \partial \eta_{k}^{c}}\left(s^{\iota}\right)\right)_{j, k=1, \ldots, n}\left({ }^{t} T_{\iota} T(\vartheta)\right), \iota=1,2,
\end{aligned}
$$

where $s^{\iota}=T_{\iota} \sigma^{\iota}, \iota=1,2$.

Thus the matrices $\left(\partial^{2} \lambda_{c}\left(T(\vartheta) \sigma^{\circ}\right) / \partial \eta_{j} \partial \eta_{k}\right)_{j, l=1, \ldots, n}$ of order $n$ are nonnegative definite and have zero as an eigenvector and $\operatorname{dim} \operatorname{ker}\left(\partial^{2} \lambda_{\iota}\left(T(\vartheta) \sigma^{\circ}\right) /\right.$ $\left.\partial \eta_{j} \partial \eta_{k}\right)_{j, k-1, \ldots, n}=1$. By the relation:

$$
\begin{aligned}
& \left(\frac{\partial^{2} \lambda}{\partial \eta_{j} \partial \eta_{k}}\left(T(\vartheta) \sigma^{\circ}, \vartheta\right)\right)_{j, k=1, \cdots, n}=\vartheta\left(\frac{\partial^{2} \lambda_{1}}{\partial \eta_{j} \partial \eta_{k}}\left(T(\vartheta) \sigma^{\circ}\right)\right)_{j, k-1, \cdots, n} \\
& \quad+(1-\vartheta)\left(\frac{\partial^{2} \lambda_{2}}{\partial \eta_{j} \partial \eta_{k}}\left(T(\vartheta) \sigma^{\circ}\right)\right)_{j, k=1, \cdots, n}
\end{aligned}
$$

the Hessian matrix of $\lambda(T \eta, \vartheta)$ (relative to $\eta$ ) at $\eta=\sigma^{\circ}$ is nonnegative definite and the dimension of its kernel is 1 . Moreover $\sigma^{\circ}$ is an eigenvector of the matrix corresponding to the eigenvalue 0 and $\sigma_{n}^{\circ} \neq 0$, for $(7 \cdot 10) \quad 1=\lambda\left(T(\vartheta) \sigma^{\circ}, \vartheta\right)=\sum_{j=1}^{n} \sigma_{j}^{\circ} \frac{\partial \lambda}{\partial \eta_{j}}\left(T(\vartheta) \sigma^{\circ}, \vartheta\right)=\sigma_{n}^{\circ} \frac{\partial \lambda}{\partial \eta_{n}}\left(T(\vartheta) \sigma^{\circ}, \vartheta\right)$.

By the second statement of Lemma 7.2 , the matrix $\left(\partial^{2} \lambda\left(T(\vartheta) \sigma^{\circ}, \vartheta\right) /\right.$ $\left.\partial \eta_{j} \partial \eta_{k}\right)_{j, k=1, \cdots, n-1}$ of order $n-1$ is positive definite. This implies that the Caussian curvature of $S(\vartheta)$ at $s^{\circ}$ is different from zero. The proof is complete.

Let us now investigate the asymptotic behavior as $|x| \rightarrow \infty$ of the integral $(\tau \cdot 2)$ under the assumption in Theorem 7.1 . It suffices to consider the integral for the case $x=\left(0, \cdots, 0, x_{n}\right)$ since it is possible to reduce the general case to this one by rotation of coordinate axes as in the proof of Theorem 5.1. We choose a finite partition of unity $\left\{\phi_{j}\right\}$ over $[0,1]: \Sigma \phi_{j}(\theta)=1$ in $[0,1]$, where $\phi_{j} \in C_{0}^{\infty}(-\delta, 1+\delta), \delta>0$ and 
the supports of all the $\phi_{j}$ are sufficiently small. Let $\left\{\varphi_{k}(\theta)\right\}$ be a sufficiently fine $C^{\infty}$ partition of unity on the unit sphere $\Omega$. We extend every $\varphi_{k}$ to the space $\mathbb{H}^{n}$ by homogeneity of degree 0 and denote it by the same letter $\varphi_{k}(\xi)$. The system $\left\{\varphi_{k}(\xi)\right\}$ forms a $C^{\infty}$ partition of unity in 思 $^{n} \backslash\{0\}$. Then the integral (7.2) is represented in the form of a sum

$$
I(x)=\sum_{j, k} \int_{0}^{1} d \vartheta \int_{S(\vartheta)} \mu_{j k}(s, \vartheta) \exp \{i\langle x, s\rangle\} d S(\vartheta)
$$

where $\mu_{j k}(s, \vartheta)=\phi_{j}(\vartheta) \varphi_{k}(s) \mu(s, \vartheta)$. By this localization, it is sufficient to consider the case when $\mu(s, \vartheta) \neq 0$ only for $\vartheta$ is a small neighborhood $\left(\vartheta^{\circ}-\delta, \vartheta^{\circ}+\delta\right) \cap[0,1]$ of a point $\vartheta^{\circ} \in[0,1]$ and only for $s$ in a small neighborhood in $\mathbb{F}^{n}$ of a point $s^{\circ} \in S\left(\vartheta^{\circ}\right)$.

First consider the case when the normal to the surface $S\left(\vartheta^{\circ}\right)$ at $s^{\circ}$ is not parallel to the vector $(0, \cdots, 0,1)$. Then we may assume that the normal to $S(\vartheta)$ at every point $s$ belonging to $\operatorname{supp}_{s} \mu(s, \vartheta)$ is never parallel to $(0, \cdots, 0,1)$ for any $\vartheta \in\left(\vartheta^{\circ}-\delta, \vartheta^{\circ}+\delta\right) \cap[0,1]$ taking $\delta$ sufficiently small if necessary. Let $s_{j}=s_{j}\left(\sigma^{\prime}, \vartheta\right), \sigma^{\prime}=\left(\sigma_{1}, \cdots, \sigma_{n-1}\right), j=1, \cdots, n$ be a system of equations which defines the part of the surface $S(\vartheta)$ containing $\operatorname{supp}_{s} \mu(s, \vartheta)$ for every $\vartheta \in\left(\vartheta^{\circ}-\delta, \vartheta^{\circ}+\delta\right) \cap[0,1]$. By assumption we have

$$
\underset{\sigma^{\prime}}{\operatorname{grad}} s_{n}\left(\sigma^{\prime}, \vartheta\right) \neq 0 \quad \text { for } \quad \vartheta \in\left(\vartheta^{\circ}-\delta, \vartheta^{\circ}+\delta\right) \cap[0,1]
$$

and for $\sigma^{\prime}$ such that $s\left(\sigma^{\prime}, \vartheta\right) \in \operatorname{supp}_{s} \mu(s, \vartheta)$. Hence successive integrations by parts give

$$
I\left(0, x_{n}\right)=O\left(x_{n}^{-\infty}\right) \quad \text { as } \quad x_{n} \rightarrow \infty \text {. }
$$

Next consider the case when the normal to the surface $s\left(\vartheta^{\circ}\right)$ at $s^{\circ}$ is parallel to the vector $(0, \cdots, 0,1)$. In this case the part of the surface $S(\vartheta)$ containing $\operatorname{supp}_{s} \mu(s, \vartheta)$ can be defined by an equation of the form $s_{n}=h\left(s^{\prime}, \vartheta\right)$ for any $\vartheta \in\left(\vartheta^{\circ}-\delta, \vartheta^{\circ}+\delta\right) \cap[0,1]$ taking $\delta>0$ smaller if necessary (see the proof of Theorem 5.1). Here $s_{n}=h\left(s^{\prime}, \vartheta\right)$ satisfies

$$
\lambda\left(s^{\prime}, h\left(s^{\prime}, \vartheta\right), \vartheta\right)=1 \quad \text { and } \quad s_{n}^{\circ}=h\left(s^{\circ \prime}, \vartheta^{\circ}\right)
$$

Consider the system of equations 


$$
a_{i}\left(s^{\prime}, \vartheta\right) \equiv \frac{\partial h}{\partial s_{j}}\left(s^{\prime}, \vartheta\right)=0, \quad j=1, \cdots, n-1 .
$$

By assumption

$$
a_{j}\left(s^{\circ}, \vartheta^{\circ}\right)=\frac{\partial h}{\partial s_{i}}\left(s^{\circ}, \vartheta^{\circ}\right)=0, \quad j=1, \cdots, n-1
$$

and by Theorem 7.1 we have

$$
\left.\frac{D\left(a_{1}, \cdots, a_{n-1}\right)}{D\left(s_{1}, \cdots, s_{n-1}\right)}\right|_{\left(s^{\circ \prime}, \vartheta^{\circ}\right)}=\operatorname{det}\left(\frac{\partial^{2} h}{\partial s_{j} \partial s_{k}}\left(s^{\circ \prime}, \vartheta^{\circ}\right)\right)=K\left(s^{\circ}, \vartheta^{\circ}\right) \neq 0 .
$$

Consequently there exists by the implicit function theorem a system of $C^{\circ}$ functions $s^{\prime}(\vartheta)=\left(s_{1}(\vartheta), \cdots, s_{n-1}(\vartheta)\right)$ defined in $\left(\vartheta^{\circ}-\delta, \vartheta^{\circ}+\delta\right) \cap[0,1]$ such that

$$
a_{j}\left(s^{\prime}(\vartheta), \vartheta\right)=\frac{\partial h}{\partial s_{j}}\left(s^{\prime}(\vartheta), \vartheta\right)=0, \quad j=1, \cdots, n-1 .
$$

Since $\operatorname{Hess}_{s^{\prime}} h\left(s^{\circ}, \vartheta^{\circ}\right)=K\left(s^{\circ}, \vartheta^{\circ}\right) \neq 0$, we may assume that the critical point $s^{\prime}(\vartheta)$ of $h\left(s^{\prime}, \vartheta\right)$ as a function of $s^{\prime}$ is non-degenerate, that is, $(7 \cdot 19) \quad \operatorname{Hesss}_{s^{\prime}} h\left(s^{\prime}(\vartheta), \vartheta\right) \neq 0$ for any $\vartheta \in\left(\vartheta^{\circ}-\delta, \vartheta^{\circ}+\delta\right) \cap[0,1]$.

Now consider the integral $(7 \cdot 2)$ with $x=\left(0, \cdots, 0, x_{n}\right)$. We can write $(7 \cdot 20) \quad I\left(0, x_{n}\right)=\int_{0}^{1} d \vartheta \int_{E^{n-1}} \mu\left(s^{\prime}, h\left(s^{\prime}, \vartheta\right), \vartheta\right)\left\{1+\left.\underset{s^{\prime}}{\operatorname{grad}} h\left(s^{\prime}, \vartheta\right)\right|^{2}\right\}^{1 / 2}$

$$
\aleph \exp \left\{i x_{n} h\left(s^{\prime}, \vartheta\right)\right\} d s^{\prime} .
$$

Since the support of $\mu\left(s^{\prime}, h\left(s^{\prime}, \vartheta\right), \vartheta\right)$ as a function of $s^{\prime}$ is compact in $\mathbb{E}^{n-1}$, we can apply $2^{\circ}$ of Theorem 4.1 to the inside integral of $(7 \cdot 20)$. The application gives

$$
\begin{aligned}
& I\left(0, x_{n}\right)=(2 \pi)^{(n-1) / 2} \exp \{-i \pi(n-1) / 4\} x_{n}^{-(n-1) / 2} \\
& \times \int_{0}^{1} \frac{\mu\left(s^{\prime}(\vartheta), h\left(s^{\prime}(\vartheta), \vartheta\right), \vartheta\right)}{\left|\operatorname{Hess}_{s^{\prime}} h\left(s^{\prime}(\vartheta), \vartheta\right)\right|^{1 / 2}}\left(1+\psi(\vartheta) x_{n}^{-1}\right) \exp \left\{i x_{n} h\left(s^{\prime}(\vartheta), \vartheta\right)\right\} d \vartheta \\
& \quad+O\left(x_{n}^{-(n+3) / 2}\right) \quad \text { as } \quad x_{n} \rightarrow \infty,
\end{aligned}
$$

where $\phi(\vartheta)$ is a $C^{\infty}$ function of $\vartheta$ in $\left(\vartheta^{\circ}-\delta, \vartheta^{\circ}+\delta\right) \cap[0,1]$. We set

$$
b(\vartheta) \equiv h\left(s^{\prime}(\vartheta), \vartheta\right) .
$$

Then 


$$
\frac{d b}{d \vartheta}\left(\vartheta^{\circ}\right)=\frac{\partial h}{\partial \vartheta}\left(s^{\circ \prime}, \vartheta^{\circ}\right)=-\frac{\lambda_{1}\left(s^{\circ}\right)-\lambda_{2}\left(s^{\circ}\right)}{\frac{\partial \lambda}{\partial s_{n}}\left(s^{\circ}, \vartheta^{\circ}\right)} .
$$

Consider the case $s^{\circ} \notin S_{1} \cap S_{2}$. In this case we have $d b\left(\vartheta^{\circ}\right) / d \vartheta \neq 0$ from $(7 \cdot 23)$. If $0<\vartheta^{\circ}<1$ we may assume from the localization $(7 \cdot 11)$ that the support of the function $\mu\left(s^{\prime}(\vartheta), h\left(s^{\prime}(\vartheta), \vartheta\right), \vartheta\right)$ of $\vartheta$ is compact and contained in the open interval $\left(\vartheta^{\circ}-\delta, \vartheta^{\circ}+\delta\right) \subset(0,1)$ with a sufficiently small $\delta>0$. Therefore we find by repeated use of integration by parts

$$
I\left(0, x_{n}\right)=O\left(x_{n}^{-\infty}\right) \quad \text { as } \quad x_{n} \rightarrow \infty .
$$

When $\vartheta^{\circ}=0$ or 1 , an integration by parts gives

$$
\begin{aligned}
I\left(0, x_{n}\right) & =i\left(1-2 \vartheta^{\circ}\right)(2 \pi)^{(n-1) / 2} \frac{\mu\left(s^{\circ}, \vartheta^{\circ}\right) \exp \left\{-i \pi(n-1) / 4+i x_{n} s_{n}^{\circ}\right\}}{\left|\operatorname{Hess}_{s^{\prime}} h\left(s^{\circ \prime}, \vartheta^{\circ}\right)\right|^{1 / 2} \frac{\partial h}{\partial \vartheta}\left(s^{\circ \prime}, \vartheta^{\circ}\right)} \\
& \times x_{n}^{-(n+1) / 2}+O\left(x_{n}^{-(n+3) / 2}\right) \text { as } x_{n} \rightarrow \infty \\
& =i(2 \pi)^{(n-1) / 2} \frac{\mu\left(s^{\circ}, \vartheta^{\circ}\right) \frac{\partial \lambda_{2-\vartheta^{\circ}}}{\partial s_{n}}\left(s^{\circ}\right)}{\left|K\left(s^{\circ}, \vartheta^{\circ}\right)\right|^{1 / 2}\left(1-\lambda_{1-\vartheta^{\circ}}\left(s^{\circ}\right)\right)} \\
& \times \exp \left\{-i \pi(n-1) / 4+i x_{n} s_{n}^{\circ}\right\} x_{n}^{-(n+1) / 2}+O\left(x_{n}^{-(n+3) / 2}\right) \\
& \text { as } x_{n} \rightarrow \infty .
\end{aligned}
$$

Consider the case $s^{\circ} \in S_{1} \cap S_{2}$. In this case we have $\partial h\left(s^{\circ \prime}, \vartheta^{\circ}\right) / \partial \vartheta$ $=0$ from $(7 \cdot 23)$ and so $\left(s^{\circ \prime}, \vartheta^{\circ}\right)$ is a critical point of $h\left(s^{\prime}, \vartheta\right)$ as a function of the variable $\left(s^{\prime}, \vartheta\right)$. Moreover we have

$$
\begin{gathered}
\frac{\partial^{2} h}{\partial \vartheta^{2}}\left(s^{\circ \prime}, \vartheta^{\circ}\right)=0 \\
\frac{\partial^{2} h}{\partial s_{j} \partial \vartheta}\left(s^{\circ \prime}, \vartheta^{\circ}\right)=-\frac{\frac{\partial \lambda_{1}}{\partial \xi_{j}}\left(s^{\circ}\right)-\frac{\partial \lambda_{2}}{\partial \xi_{j}}\left(s^{\circ}\right)}{\frac{\partial \lambda}{\partial \xi_{n}}\left(s^{\circ}, \vartheta^{\circ}\right)} \neq 0 \\
\text { for some } j(1 \leq j \leq n-1) .
\end{gathered}
$$

In fact, $(7 \cdot 27)$ follows from the assumption ( $\lambda$. iii) in $\S 6$ and Euler's identities for the homogeneous functions $\lambda_{1}$ and $\lambda_{2}$. Denote by 
$H=H\left(s^{\circ \prime}, \vartheta^{\circ}\right)$ the Hessian matrix $\left(\partial^{2} h\left(s^{\circ \prime}, \vartheta^{\circ}\right) / \partial s_{j} \partial s_{k}\right)_{j, k-1, \cdots, n-1}$ of $h\left(s^{\prime}, \vartheta^{\circ}\right)$ at $s^{\circ}$ as a function of $s^{\prime}$ and by $\mathfrak{h}=\mathfrak{h}\left(s^{\circ}, \vartheta^{\circ}\right)$ the $\operatorname{vector}^{t}\left(-\partial\left(\lambda_{1}-\lambda_{2}\right)\left(s^{\circ}\right) /\right.$ $\left.\partial s_{1}, \cdots,-\partial\left(\lambda_{1}-\lambda_{2}\right)\left(s^{\circ}\right) / \partial s_{n-1}\right) /\left(\partial \lambda\left(s^{\circ}, \vartheta^{\circ}\right) / \partial s_{n}\right)$. Since $\operatorname{det} H=K\left(s^{\circ}, \vartheta^{\circ}\right) \neq 0$ by Theorem 7.1 and $\mathfrak{h} \neq 0$ by $(7 \cdot 27)$, we have

$(7 \cdot 28) \quad \underset{\left(s^{\prime}, \vartheta\right)}{\operatorname{Hess}} h\left(s^{\circ \prime}, \vartheta^{\circ}\right)=\operatorname{det}\left(\begin{array}{cc}H & \mathfrak{h} \\ \mathfrak{h} & 0\end{array}\right)=-(\operatorname{det} H)\left\langle H^{-1} \mathfrak{h}, \mathfrak{h}\right\rangle \neq 0$.

Thus the point $\left(s^{\circ}, \vartheta^{\circ}\right)$ is a non-degenerate critical one of $h\left(s^{\prime}, \vartheta\right)$ as a function of $\left(s^{\prime}, \vartheta\right)$. Since every non-degenerate critical point is isolated, we may assume that the support of $\mu\left(s^{\prime}, h\left(s^{\prime}, \vartheta\right), \vartheta\right)$ contains no other critical points of $h\left(s^{\prime}, \vartheta\right)$ than $\left(s^{\circ \prime}, \vartheta^{\circ}\right)$. We note that on $S_{1} \cap S_{2}$ there exists only a finite number of points $s$ at which the normal to $S(\vartheta)$ is parallel to the vector $(0, \cdots, 0,1)$ for some $\vartheta \in[0,1]$ (depending on $s)$. Now we know that if the normal to $S\left(\vartheta^{\circ}\right)$ at $s^{\circ} \in S_{1} \cap S_{2}^{4}$ is parallel to $(0, \cdots, 0,1), \vartheta^{\circ}$ is a critical point of $b(\vartheta)=h\left(s^{\prime}(\vartheta), \vartheta\right)$. So let us show that the critical point is non-degenerate. In view of $(7 \cdot 26)$ we have

$$
\frac{d^{2} b}{d \vartheta^{2}}\left(\vartheta^{\circ}\right)=\left\langle\mathfrak{h}, \frac{d s^{\prime}}{d \vartheta}\left(\vartheta^{\circ}\right)\right\rangle
$$

On the other hand, differentiating the both sides of $(7 \cdot 18)$ with respect to $\vartheta$, we get

$$
\sum_{h=1}^{n-1} \frac{\partial a_{j}}{\partial s_{k}}\left(s^{\prime}(\vartheta), \vartheta\right) \frac{d s_{j}}{d \vartheta}(\vartheta)+\frac{\partial a_{j}}{\partial \vartheta}\left(s^{\prime}(\vartheta), \vartheta\right)=0, j=1, \cdots, n-1,
$$

and so

$$
\frac{d s^{\prime}}{d \vartheta}\left(\vartheta^{\circ}\right)=-H^{-1} \mathfrak{h}
$$

Substituting $(7 \cdot 30)$ in $(7 \cdot 29)$, we have

$$
\frac{d^{2} b}{d \vartheta^{2}}\left(\vartheta^{\circ}\right)=-\left\langle H^{-1} \mathfrak{h}, \mathfrak{h}\right\rangle=\frac{\underset{\left(s^{\prime}, \vartheta\right)}{\operatorname{Hess}} h\left(s^{\circ \prime}, \vartheta^{\circ}\right)}{\underset{s^{\prime}}{\operatorname{Hess}} h\left(s^{\circ \prime}, \vartheta^{\circ}\right)} \neq 0 .
$$

Hence, if $0<\vartheta^{\circ}<1$ we can apply $2^{\circ}$ of Theorem 4.1 (for $m=1$ ) to the integral $(7 \cdot 21)$ with respect to $\vartheta$. This gives

$$
I\left(0, x_{n}\right)=(2 \pi)^{n / 2} \frac{\mu\left(s^{\circ}, \vartheta^{\circ}\right)}{\left|K\left(s^{\circ}, \vartheta^{\circ}\right)\left\langle H^{-1} \mathfrak{h}, \bar{h}\right\rangle\right|^{1 / 2}}
$$




$$
\begin{gathered}
\times \exp \left\{-i \pi n / 4+i x_{n} s_{n}{ }^{\circ}\right\} x_{n}^{-n / 2}+O\left(x_{n}^{-(n+2) / 2}\right) \\
\text { as } x_{n} \rightarrow \infty .
\end{gathered}
$$

The asymptotic formula can also be obtained applying directly $2^{\circ}$ of Theorem 4.1 to the integral $(7 \cdot 20)$ with respect to $\left(s^{\prime}, \vartheta\right)$. If $\vartheta^{\circ}=0$ we apply Theorem 4.2 to the integral $(7 \cdot 21)$. Then we find

$$
\begin{gathered}
I\left(0, x_{n}\right)=\frac{1}{2}(2 \pi)^{n / 2} \frac{\mu\left(s^{\circ}, 0\right)}{\left|K\left(s^{\circ}, 0\right)\left\langle H^{-1} \mathfrak{h}, \mathfrak{h}\right\rangle\right|^{1 / 2}} \\
\times \exp \left\{-i \pi n / 4+i x_{n} s_{n}{ }^{\circ}\right\} x_{n}^{-n / 2}+O\left(x_{n}^{-(n+1, / 2}\right) \\
\text { as } \quad x_{n} \rightarrow \infty .
\end{gathered}
$$

The case $\vartheta^{\circ}=1$ can be reduced to this one by the substitution of $\vartheta$ for $1-\vartheta$ in the integral $(7 \cdot 21)$. Summing up, we have proved

Theorem $7_{0}$ 马. Let $\lambda_{1}(\xi)$ and $\lambda_{2}(\xi)$ be real valued functions : $^{n}$ satisfying the conditions ( $\lambda . \mathrm{i}),(\lambda . \mathrm{ii})$ and ( $\lambda . \mathrm{iii})$ in $\S 6$ and the assumption in Theorem 7.1. Sct $\lambda(\xi, \vartheta)=\vartheta \lambda_{1}(\xi)+(1-\vartheta) \lambda_{2}(\xi)$ and $S(\vartheta)=\{s ; \lambda(s, \vartheta)=1\}, 0 \leq \vartheta \leq 1$. Let $I(x)$ be the integral defined by $(7 \cdot 2)$ and let $\theta$ be a given unit vector. If there exist points $s$ on $S_{1} \cap S_{2}$ at which the normal to $S(\vartheta)$ for some $\vartheta \in[0,1]$ (the $\vartheta$ depending on the point $s$ ) is parallel to $\theta$, let $s^{1}(\theta), \cdots, s^{r}(\theta)$ be all such points and $\vartheta^{1}, \cdots, \vartheta^{r}$ the corresponding values of the parameter $\vartheta$. Then the asymptotic behavior of $I(x)$ for $|x| \rightarrow \infty$ along the ray $x=|x| \theta$ is given by

$$
I(x)=\sum_{\ell=1}^{r} C\left(\theta, s^{\iota}(\theta), \vartheta^{\iota}\right) \exp \left\{i|x|\left\langle\theta, s^{\iota}(\theta)\right\rangle\right\}|x|^{-n / 2}+q(x),
$$

where

$$
C(\theta, s, \vartheta)=\left\{\begin{array}{c}
\frac{(2 \pi)^{n / 2} \mu(s, \vartheta) \exp \{-i \pi n / 4\}}{\left|K(s, \vartheta)\left\langle H^{-1}(s, \vartheta) \mathfrak{h}(\theta, s, \vartheta), \mathfrak{h}(\theta, s, \vartheta)\right\rangle\right|^{1 / 2}}- \\
\text { if } 0<\vartheta<1, \\
\frac{(2 \pi)^{n / 2} \mu(s, \vartheta) \exp \{-i \pi n / 4\}}{2\left|K(s, \vartheta)\left\langle H^{-1}(s, \vartheta) \mathfrak{h}(\theta, s, \vartheta), \mathfrak{h}(\theta, s, \vartheta)\right\rangle\right|^{1 / 2}} \\
\text { if } \vartheta=0 \text { or } 1
\end{array}\right.
$$

and for each multi-index $\nu$ 


$$
(\partial / \partial x)^{\nu} q(x)=O\left(|x|^{-(n+1) / 2}\right) \text { for }|x| \rightarrow \infty \text { along } x=|x| \theta \text {. }
$$

In the formula $(7 \cdot 35), K(s, \vartheta)$ is the Gaussian curvature of $S(\vartheta)$ at $s \in S_{1} \cap S_{2}, H(s, \vartheta)$ denotes the Weigarten operator in the tangent space of $S(\vartheta)$ at the point $s$ and $\mathfrak{h}(\theta, s, \vartheta)$ denotes the orthogonal projection of the vector $\underset{\xi}{\operatorname{grad}}\left(\lambda_{1}-\lambda_{2}\right)(s) /\langle\operatorname{grad} \lambda(s, \vartheta), \theta\rangle$ on the tangent hyperplane of $S(\vartheta)$ at the point $s$.

Next suppose that for any point $s \in S_{1} \cap S_{2}$ and any $\vartheta \in[0,1]$ the normal to $S(\vartheta)$ at $s$ is never parallel to $\theta$. Let $s_{k}(\theta)$ be the inverse image of $\theta$ under the Gauss map $S_{k} \ni s \mapsto n_{k}(s) \in \Omega$ for $k=1,2$. Then the asymptotic behavior of $I(x)$ for $|x| \rightarrow \infty$ along the ray $x=|x| \theta$ is given by

$$
\begin{aligned}
I(x)= & i(2 \pi)^{(n-1) / \Omega} \sum_{k=1}^{2}\left[\frac{\mu\left(s_{k}(\theta), 2-k\right)\left\langle\operatorname{grad} \lambda_{k}\left(s_{k}(\theta), \theta\right)\right\rangle}{\left|K\left(s_{k}(\theta)\right)\right|^{1 / 2}\left(1-\lambda_{3-k}\left(s_{k}(\theta)\right)\right)}\right. \\
& \times \exp \left\{-i \pi(n-1) / 4+i\left\langle x, s_{k}(\theta)\right\rangle\right\} \\
& +\frac{\mu\left(s_{k}(-\theta), 2-k\right)\left\langle\operatorname{grad} \lambda_{k}\left(s_{k}(-\theta)\right),-\theta\right\rangle}{\left|K\left(s_{k}(-\theta)\right)\right|^{1 / 2}\left(1-\lambda_{3-k}\left(s_{k}(-\theta)\right)\right)} \\
& \times \exp \left\{i \pi(n-1) / 4+i\left\langle x, s_{k}(-\theta)\right\rangle\right\}|x|^{-(n+1) / 2}+q(x),
\end{aligned}
$$

wherc for each multi-index $\nu$

$$
(\partial / \partial x)^{\nu} q(x)=O\left(|x|^{-(n+3) / 2}\right) \quad \text { for } \quad|x| \rightarrow \infty \text { along } x=|x| \theta .
$$

Applying Theorem 7.3 to the inner integral of the last expression of $(7 \cdot 1)$ and repeating the same argument as for $\Phi(x ; \sigma \pm i \varepsilon)$, we obtain

Theorem 7.4. Let $\Psi(x ; \sigma \pm i \varepsilon)$ be the function defined by $(6 \cdot 10)$. Let $\sigma^{\circ}$ be an arbitrarily fixed positive number and $\delta$ a small positive number such that $\left[\sigma^{\circ}-4 \delta, \sigma^{\circ}+4 \delta\right]$ does not contain 0 . Under the same assumptions as in Theorem 7.2 , the asymptotic behavior $\Psi(x ; \sigma \pm i \varepsilon)$ for $|x| \rightarrow \infty$ is described by the formulas given below.

The case where there exist points $s$ on $S_{1} \cap S_{2}$ at which the normal to $S(\vartheta)$ for some $\vartheta \in[0,1]$ is parallel to a given unit vector $\theta$ : Let $s^{1}(\theta), \cdots, s^{r}(\theta)$ be all such points and $\vartheta^{1}, \cdots, \vartheta^{r}$ the corresponding values of the parameter. Then 


$$
(\partial / \partial x)^{\nu} \Psi(x ; \sigma \pm i \varepsilon)=\underset{\iota}{\sum_{t}} C_{ \pm, \nu}\left(\theta, s^{\iota}(\theta), \vartheta^{\natural}\right)|x|^{-n / 2+1}
$$

$$
\times I_{\nu, s i(\theta)}(x ; \sigma \pm i \varepsilon)+O\left(|x|^{-n / 2+1-r}\right)
$$

for $|x| \rightarrow \infty$ along the ray $x=|x| \theta, \sigma \pm i \varepsilon \in \Delta_{ \pm}, 0<\gamma<1 / 2$,

$(7 \cdot 40) \quad C_{ \pm, \nu}(\theta, s, \vartheta)$

$$
=\left\{\begin{array}{c}
\frac{(2 \pi)^{n / 2}(i s)^{\nu} P(s, \vartheta) \exp \{\mp i \pi n / 4\}}{\left|K(s, \vartheta)\left\langle H^{-1}(s, \vartheta) \mathfrak{h}(\theta, s, \vartheta), \mathfrak{h}(\theta, s, \vartheta)\right\rangle\right|^{1 / 2}|\operatorname{grad} \lambda(s, \vartheta)|} \\
\text { if } 0<\vartheta<1, \\
\frac{(2 \pi)^{n / 2}(i s)^{\nu} P(s, \vartheta) \exp \{\mp i \pi n / 4\}}{2\left|K(s, \vartheta)\left\langle H^{-1}(s, \vartheta) \mathfrak{h}(\theta, s, \vartheta), \mathfrak{h}(\theta, s, \vartheta)\right\rangle\right|^{1 / 2}|\operatorname{grad} \lambda(s, \vartheta)|} \\
\text { if } \vartheta=0 \text { or } 1
\end{array}\right.
$$

and

$$
I_{\nu, s}(x ; \sigma \pm i \varepsilon)=|x|^{-1} \int_{0}^{\infty} \frac{\rho^{n / 2-1+d+|\nu|} \psi(\rho)}{(\rho-(\sigma \pm i \varepsilon))^{2}} \exp \{i \rho\langle x, s\rangle\} d \rho
$$

where the summation $\sum_{c}$ in $(7 \cdot 39)$ is taken over c such that $\left\langle\theta, s^{c}(\theta)\right\rangle>0$ or the $\left\langle\theta, s^{\ell}(\theta)\right\rangle\langle 0$ according as $\sigma+i \varepsilon$ or $\sigma-i \varepsilon$ and the order relation $O$ in (7.39) is uniform with respect to $\sigma \pm i \varepsilon \in A_{ \pm}$but depends on $\gamma$. Further $I_{\nu, s^{2}(\theta)}(x ; \sigma \pm i \varepsilon)$ are bounded continuous functions in $\{x ;|x|$ $\geq 1\} \times \Delta_{ \pm}$. In particular,

$$
\begin{gathered}
(\partial / \partial x)^{\nu} \Psi(x ; \sigma \pm i 0)=\mp 2 \pi \sigma^{n / 2-1+d+|\nu|} \sum_{\iota} C_{ \pm, \nu}\left(\theta, s^{\iota}(\theta), \vartheta^{\iota}\right) \\
\quad \times\left\langle\theta, s^{\iota}(\theta)\right\rangle \exp \left\{i \sigma\left\langle x, s^{\iota}(\theta)\right\rangle\right\}|x|^{-n / 2+1}+O\left(|x|^{-n / 2+1-r}\right) \\
\quad \text { for } \quad|x| \rightarrow \infty \text { along the ray } x=|x| \theta,\left|\sigma-\sigma^{\circ}\right| \leq \delta .
\end{gathered}
$$

The case where for any point $s \in S_{1} \cap S_{2}$ and any $\vartheta \in[0,1]$ the normal to $S(\vartheta)$ at $s$ is never parallel to $\theta$ : Let $s_{k}(\theta)$ be the inverse image of $\theta$ under the Gauss map: $S_{k} \ni s \mapsto n(s) \in \Omega$ where $n(s)$ is the outward unit normal to $S_{k}$ at $s$ and $\Omega$ is the unit sphere. Then we have

$$
\begin{aligned}
& (\partial / \partial x)^{\nu} \Psi(x ; \sigma \pm i \varepsilon)=i(2 \pi)^{(n-1) / 2} \exp \{\mp i \pi(n-1) / 4\} \\
& \quad \times \sum_{k=1}^{2} \frac{\left(i s_{k}( \pm \theta)\right)^{\nu} P_{k}\left(s_{k}( \pm \theta)\right)\left\langle\operatorname{grad} \lambda_{k}\left(s_{k}( \pm \theta)\right), \pm \theta\right\rangle}{\left|K\left(s_{k}( \pm \theta)\right)\right|^{1 / 2}\left|\operatorname{grad} \lambda_{k}\left(s_{k}( \pm \theta)\right)\right|\left\{1-\lambda_{3-k}\left(s_{k}( \pm \theta)\right)\right\}} \\
& \quad \times|x|^{-(n-1) / 2} I_{\nu, s_{k}( \pm \theta)}(x ; \sigma \pm i \varepsilon)+O\left(|x|^{-(n-1) / 2-r}\right)
\end{aligned}
$$


and

$$
I_{\nu, s}(x ; \sigma \pm i \varepsilon)=|x|^{-1} \int_{0}^{\infty} \frac{\rho^{(n-3) / 2+d+|\nu|} \phi(\rho)}{(\rho-(\sigma \pm i \varepsilon))^{2}} \exp \{i \rho\langle x, s\rangle\} d \rho,
$$

where the order relation $O$ in $(7.43)$ is uniform with respect to $\sigma \pm i \varepsilon \in \Delta_{ \pm}$but depends on $\gamma(0<\gamma<1)$ and $I_{\nu, s_{k}( \pm \theta)}(x ; \sigma \pm i \varepsilon)$ are bounded continuous functions in $\{x ;|x| \geq 1\} \times \Delta_{ \pm}$. In particular,

$$
\begin{aligned}
& (\partial / \partial x)^{\nu} \Psi(x ; \sigma \pm i 0)=\mp(2 \pi)^{(n+1) / 2} i \sigma^{(n-3) / 2+d+|\nu|} \\
& \times \sum_{k=1}^{2} \frac{\left(i s_{k}( \pm \theta)\right)^{\nu} P_{k}\left(s_{k}( \pm \theta)\right)\left\langle\operatorname{grad} \lambda_{k}\left(s_{k}( \pm \theta)\right), \pm \theta\right\rangle\left\langle\theta, s_{k}( \pm \theta)\right\rangle}{\left|K\left(s_{k}( \pm \theta)\right)\right|^{1 / 2}\left|\operatorname{grad} \lambda_{k}\left(s_{k}( \pm \theta)\right)\right|\left\{1-\lambda_{3-k}\left(s_{k}( \pm \theta)\right)\right\}} \\
& \times \exp \left\{\mp i \pi(n-1) / 4+i \sigma\left\langle x, s_{k}( \pm \theta)\right\rangle\right\}|x|^{-(n-1) / 2} \\
& +O\left(|x|^{-(n-1) / 2-r}\right) \\
& \quad \text { for }|x| \rightarrow \infty \text { along the ray } x=|x| \theta,\left|\sigma-\sigma^{0}\right| \leq 0 .
\end{aligned}
$$

\section{§. Formulas for the Asymptotic Behavior of Green's Functions at Infinity}

In this section we shall give formulas for the asymptotic behavior as $|. x| \rightarrow \infty$ of the Green's functions of the operators $A-\lambda I$ which satisfy the conditions i), ii) and iv), or the conditions $1^{\circ}, 2^{\circ}, 3^{\circ}, 4^{\circ}$ and $5^{\circ}$ in $\S 1$.

Under the conditions i), ii) and iv), we have

Theorem 8. 1. The Green's function $G(x, \lambda)$ of $A-\lambda I$ defined by (1.4) has the following properties:

(G. 1$) \quad G(x ; \lambda)$ is $C^{\cdots}$ with respect to $x$ in $\mathbf{R}^{n} \backslash\{0\}$ for each fixed $\lambda \in \mathbf{C} \backslash \mathbf{R}$ and analytic with respect to $\lambda$ in $\mathbf{C} \backslash \mathbf{R}$ for each fixed $x \in \mathbb{R}^{n} \backslash\{0\}$. Morcover, for every multi-index $\nu,(\partial / \partial x)^{\nu} G(x ; \lambda)$ is continuous in $\left(\mathbf{R}^{n} \backslash\{0\}\right) \times(\mathbf{C} \backslash \mathbf{R})$.

(G. 2) For each multi-index $\nu$ and each $(x, \sigma) \in\left(\mathbb{R}^{n} \backslash\{0\}\right)$ $\times(\mathbf{R} \backslash\{0\})$ the limits

$$
G^{(\nu)}(x ; \sigma \pm i 0)=\lim _{\varepsilon \rightarrow 0 \perp}(\partial / \partial x)^{\nu} G(x ; \sigma \pm i \varepsilon)
$$


exist and the convergence is uniform on every compact subset of $\mathbb{R}^{n} \backslash\{0\}$. Further $G^{(\nu)}(x ; \sigma \pm i 0)$ are continuous functions of $(x, \sigma)$ in $\left(\mathbb{R}^{n} \backslash\{0\}\right) \times(\mathbb{R} \backslash\{0\})$.

(G.3) Let $\sigma^{\circ}$ be an arbitrarily fixed non zero real number. We denote by $\Delta_{ \pm}$the set $\left\{\lambda=\sigma \pm i \varepsilon ;\left|\sigma-\sigma^{\circ}\right| \leq 0,0 \leq \varepsilon \leq \varepsilon^{\circ}\right\}$ and by $\Delta$ the set $\left\{\lambda ;\left|\operatorname{Re} \lambda-\sigma^{\circ}\right| \leq \delta,|\operatorname{Im} \lambda| \leq \varepsilon^{\circ}\right\}$. If we choose the positive number $\delta$ suitably small, the Green's functions $G(x ; \sigma \pm i \varepsilon)$ in $\mathbb{R}^{n} \times \Delta_{ \pm}$have a decomposition of the form:

$$
G(x ; \sigma \pm i \varepsilon)=\sum_{k=1}^{[m / 2]} G_{l k}(x ; \sigma \pm i \varepsilon)+F(x ; \sigma \pm i \varepsilon),
$$

where for any multi-index $\nu$ and for any $r$ with $0<\gamma<1$, the functions $G_{k}(x ; \sigma \pm i \varepsilon)$ have the asymptotic behavior:

$$
\begin{aligned}
& (\partial / \partial x)^{\nu} G_{k}(x ; \sigma \pm i \varepsilon)=(\operatorname{sgn} \sigma)(-1)^{\alpha_{k}-1}(2 \pi)^{-(n+1) / 2} \\
& \times \frac{\left(i s_{k}( \pm \theta) \operatorname{sgn} \sigma\right)^{\nu}\left[Q_{k}\left(s_{k}( \pm \theta)\right)\right]^{\alpha_{k}-1} P_{k}\left(s_{k}( \pm \theta)\right)}{\left|K\left(s_{k}( \pm \theta)\right)\right|^{1 / 2}\left|\operatorname{grad} \lambda_{k}\left(s_{k}( \pm \theta)\right)\right|} \\
& \quad \times \exp \{\mp i \pi(n-1) \operatorname{sgn} \sigma / 4\} \\
& \quad \times I_{\alpha_{k}-1, \nu, s_{k}( \pm \theta)}((\operatorname{sgn} \sigma) x ;|\sigma| \pm i \varepsilon \operatorname{sgn} \sigma)|x|^{-(n-1) / 2+\alpha_{k}-1} \\
& +O\left(|x|^{-(n-1) / 2+\alpha_{k}-1-r}\right) \\
& \quad \text { for } \quad|x| \rightarrow \infty, x=|x| \theta, \sigma \pm i \varepsilon \in \Delta_{ \pm}, 0<\gamma<1 .
\end{aligned}
$$

Here $s_{k}(\theta)$ denotes the inverse of the Gauss map: $S_{k} \rightarrow \Omega, P_{k}(\xi)$ and $Q_{k}(\xi)$ are the matrices defined by $(3 \cdot 7)$ and $(3 \cdot 14)$ respectively, and

$$
I_{\alpha_{k}-1, \nu, s}(x ; \sigma \pm i \varepsilon)=|x|^{1-\alpha_{k}} \int_{0}^{\infty} \frac{\rho^{(n-1) / 2+\alpha_{k}-1+|\nu|} \psi(\rho)}{(\rho-(\sigma \pm i \varepsilon))^{\alpha_{k}}} \exp \{i \rho\langle x, s\rangle\} d \rho,
$$

where $\psi$ is a suitable function such that $\phi \in C_{\circ}{ }^{\infty}(0, \infty)$ and $\phi(\rho)=1$ for $\left|\rho-\sigma^{\circ}\right| \leq \delta$. The order relation $O$ in (8.3) is uniform with respect to $\sigma \pm i \varepsilon \in \Delta_{ \pm}$and $\theta=x /|x| \in \Omega$ but depends on $\gamma$. The functions $I_{\alpha_{k}-1, \nu, s_{k}( \pm \theta)}(x ;|\sigma| \pm i \varepsilon)$ are bounded continuous in $\{x ;|x| \geq 1\} \times \Delta_{ \pm}$. For $\varepsilon=0$, the functions $(\partial / \partial x)^{\nu} G_{k}(x ; \sigma \pm i \varepsilon)$ have, in particular, the form:

$$
\begin{aligned}
& (\partial / \partial x)^{\nu} G_{k}(x ; \sigma \pm i 0)=\frac{ \pm i(-1)^{\alpha_{k}-1}(2 \pi)^{-(n-1) / 2}\left(i s_{k}( \pm \theta) \operatorname{sgn} \sigma\right)^{\nu}}{\left(\alpha_{k}-1\right) !\left|K\left(s_{k}( \pm \theta)\right)\right|^{1 / 2}} \\
\times & \frac{\left(i\left\langle\theta, s_{k}( \pm \theta)\right\rangle Q_{k}\left(s_{k}( \pm \theta)\right) \operatorname{sgn} \sigma\right)^{\alpha_{k}-1} P_{k}\left(s_{k}( \pm \theta)\right)}{\mid \operatorname{grad} \lambda_{k}\left(s_{k}( \pm \theta)\right) !}|\sigma|^{(n-1) / 2+\alpha_{k}-1+|\nu|}
\end{aligned}
$$




$$
\begin{aligned}
& \times \exp \left\{\mp i \pi(n-1) \operatorname{sgn} \sigma / 4+i \sigma\left\langle x, s_{k}( \pm \theta)\right\rangle\right\}|x|^{-(n-1) / 2+\alpha_{k}-1} \\
& +O\left(|x|^{-(n-1) / 2+\alpha_{k}-1-\tau}\right) \text { for } \quad|x| \rightarrow \infty, x=|x| \theta,\left|\sigma-\sigma^{\circ}\right| \leq \delta .
\end{aligned}
$$

Further we have for some positive numbers $C_{\lrcorner_{\Perp}, \alpha_{k}, r, \nu}$ and $R_{\lrcorner_{k}, \alpha_{k}}$

$$
\begin{aligned}
& \left|(\partial / \partial x)^{\nu}\left\{G_{k}(x ; \sigma \pm i \varepsilon)-G_{k}\left(x ; \sigma^{\prime} \pm i \varepsilon\right)\right\}\right| \\
& \leq C_{\lrcorner_{\lrcorner}, \alpha_{k}, \gamma, \nu}\left|\sigma-\sigma^{\prime}\right| \gamma|x|^{-(n-3) / 2+\alpha_{k}-1} \\
& \quad \text { when }|x| \geq R_{\lrcorner_{\star}, \alpha_{k}} \sigma, \sigma^{\prime} \in\left[\sigma^{\circ}-\delta, \sigma^{\circ}+\delta\right] \\
& \text { and } 0 \leq \varepsilon \leq \varepsilon^{\circ} .
\end{aligned}
$$

$F(x ; \lambda) \quad(\lambda=\sigma \pm i \varepsilon)$ in the decomposition $(8 \cdot 2)$ is a distribution of $x$ in $\mathbb{R}^{n}$ and $a C^{\omega}$ function of $x$ in $\mathbb{R}^{n} \backslash\{0\}$ for each fixed $\lambda \in \Delta$ and analytic with respect to $\lambda$ for each fixed $x \in \mathbb{R}^{n} \backslash\{0\}$. Moreover we have for any multi-index $\nu$

$$
(\partial / \partial x)^{\nu} F(x ; \lambda)=O\left(|x|^{-\infty}\right) \text { as }|x| \rightarrow \infty,
$$

where the order relation $O$ is uniform with respect to $\lambda$ in 1 .

Proof. We first choose $\delta>0$ so that the interval $\left[\sigma^{\circ}-4 \delta, \sigma^{\circ}+4 \delta\right]$ does not contain zero and next a function $\phi \in C_{\circ}{ }^{\infty}\left(\Xi^{1}\right)$ with $\phi(\rho)=1$ for $|\rho-| \sigma^{\circ}|| \leq 3 \delta$ and we define the functions $\chi_{k} \in C_{\circ}{ }^{\infty}\left(\mathbb{G}^{n} \backslash\{0\}\right), k=1, \cdots$, $b=[m / 2]$ by

$$
\chi_{k}(\xi)=\chi_{k}(\rho s)=\psi(\rho), 0<\rho<+\infty, s \in S_{k}=\left\{s ; \lambda_{k}(s)=1\right\},
$$

where $(\rho, s)$ are the polar coordinates associated with $\lambda_{k}$ (or $S_{k}$ ). Further take $\psi_{0} \in C_{\circ}{ }^{\omega}\left(\Xi^{n}\right)$ which is 1 in a small neighborhood of the origin. If $\delta$ is small, the sets supp $\chi_{0}, \cdots$, supp $\chi_{b}$ are pairwise disjoint.

Now we recall that we have the spectral representation $(3 \cdot 21)$ of $(A(\hat{\xi})-\lambda I)^{-1}$ under the conditions i), ii) and iv) in $\S 1$. We consider only the case where $m$ is odd, since the even case can be proved in the same way by obvious modifications. Taking account of $(3 \cdot 21)$ and making use of the $\chi_{k}(\xi)$, we decompose $(A(\xi)-\lambda I)^{-1}$ with $\lambda=\sigma \pm i \varepsilon,\left|\sigma-\sigma^{\circ}\right|$ $\leq 20,0<\varepsilon \leq \varepsilon^{\circ}$ in the following way.

Case: $\sigma^{\circ}>0$ and $m=2 b+1$

$$
\begin{gathered}
(A(\xi)-\lambda I)^{-1}=\sum_{k=1}^{b} \chi_{k}(\xi)\left\{\sum_{j=0}^{a_{k}-1}(-1)^{j}\left(\lambda_{k}(\xi)-\lambda\right)^{-j-1}\right. \\
\left.\times\left(Q_{k}(\xi)\right)^{j} P_{k}(\xi)\right\}+R(\xi, \lambda),
\end{gathered}
$$


where

$$
\begin{aligned}
(8 \cdot 10) \quad R(\xi, \lambda) & =\chi_{\circ}(\xi)(A(\xi)-\lambda I)^{-1}-\left(1-\chi_{0}(\xi)\right) \sum_{j=0}^{a_{b+1}-1}\left(Q_{b+1}(\xi)\right)^{j} \lambda^{-j-1} \\
& +\left(1-\chi_{0}(\xi)\right) \sum_{k=1}^{b}\left(1-\chi_{k}(\xi)\right)\left\{\sum_{j=0}^{\alpha_{k}-1}(-1)^{j}\left(\lambda_{k}(\xi)-\lambda\right)^{-j-1}\right. \\
& \left.\times\left(Q_{k}(\xi)\right)^{j}\right\} P_{k}(\xi)+\left(1-\chi_{0}(\xi)\right) \sum_{k=b+2}^{m}\left\{\sum_{j=0}^{a_{k}-1}(-1)^{j}\right. \\
& \left.\times\left(\lambda_{k}(\xi)-\lambda\right)^{-j-1}\left(Q_{k}(\xi)\right)^{j}\right\} P_{k}(\xi) .
\end{aligned}
$$

Case: $\sigma^{\circ}<0$ and $m=2 b+1$

$$
\begin{aligned}
& (A(\xi)-\lambda I)^{-1}=\sum_{k=b+2}^{m} \chi_{m-k+1}(-\xi)\left\{\sum_{j=0}^{a_{k}-1}(-1)^{j}\left(\lambda_{k}(\xi)-\lambda\right)^{-j-1}\right. \\
& \left.\times\left(Q_{k}(\xi)\right)^{j}\right\} P_{k}(\xi)+R(\xi, \lambda),
\end{aligned}
$$

where

$$
\begin{aligned}
(8 \cdot 12) \quad R(\xi, \lambda) & =\psi_{0}(\xi)(A(\xi)-\lambda I)^{-1}-\left(1-\chi_{\circ}(\xi)\right) \sum_{j=0}^{a_{b+1}-1}\left(Q_{b+1}(\xi)\right)^{j} \lambda^{-j-1} \\
& +\left(1-\chi_{0}(\xi)\right) \sum_{k=b+2}^{m}\left(1-\chi_{m-k+1}(-\xi)\right)\left\{\sum_{j=0}^{a_{k}-1}(-1)^{j}\right. \\
& \left.\times\left(\lambda_{k}(\xi)-\lambda\right)^{-j-1}\left(Q_{k}(\xi)\right)^{j}\right\} P_{k}(\xi) \\
& +\left(1-\chi_{0}(\xi)\right) \sum_{k=1}^{b}\left\{\sum_{j=0}^{a_{k}-1}(-1)^{j}\left(\lambda_{k}(\xi)-\lambda\right)^{-j-1}\left(Q_{k}(\xi)\right)^{j}\right\} P_{k}(\xi) .
\end{aligned}
$$

Making use of the relations $(3 \cdot 3),(3 \cdot 11)$ and $Q_{k}(-\xi)=-Q_{m-k+1}(\xi)$, we find that for the case $\sigma^{\circ}<0,(A(\xi)-\lambda I)^{-1}$ may be represented in the form.

$(8 \cdot 13) \quad(A(\xi)-\lambda I)^{-1}=\sum_{k=1}^{b} \chi_{k}(-\xi)\left\{\sum_{j=0}^{a_{k}-1}(-1)^{j+1}\left(\lambda_{k}(-\xi)+\lambda\right)^{-j-1}\right.$

$$
\left.\times\left(Q_{k}(-\xi)\right)^{j}\right\} P_{k}(-\xi)+R(\xi, \lambda)
$$

Define the functions $G_{k}, k=1, \cdots, b$ by

$(8 \cdot 14) \quad G_{k}(x ; \sigma \pm i \varepsilon)=\left\{\begin{array}{c}\left.\times\left(Q_{k}(\xi)\right)^{j} P_{k}(\xi)\right](x) \quad \text { if } \quad \sigma^{\circ}>0, \\ \mathscr{F}^{-1}\left[\chi_{k}(\xi) \sum_{j=0}^{\alpha_{k}-1}(-1)^{j+1}\left(\lambda_{k}(\xi)-(-\sigma \mp i \varepsilon)\right)^{-j-1}\right. \\ \left.\times\left(Q_{k}(\xi)\right)^{j} P_{k}(\xi)\right](-x) \quad \text { if } \quad \sigma^{\circ}<0,\end{array}\right.$ 
and the distribution $F$ by

$$
F(x ; \lambda)=\mathscr{F}^{-1}[R(\xi, \lambda)](x) .
$$

Applying Theorem 6.3 to $G_{k}$ we get $(8 \cdot 3)$ with $(8 \cdot 4),(8 \cdot 5)$ and (8.6). To complete the proof of Theorem 8.1 it suffices to prove the following elementary lemma.

Lemma 8.2. Let $T_{\lambda}$ be a temperate distribution valued function of $\lambda$ defined in a domain $D$ of the complex plane $\mathrm{C}$ and assume that the Fourier transform $\widehat{T}_{\lambda}(\xi)$ of $T_{\lambda}$ with respect to $x$ satisfies the conditions:

(T. 1) For every multi-index $\nu,(\partial / \partial \xi)^{n} T_{\lambda}(\xi)$ is a continuous function of $(x, \lambda)$ in $\mathbb{S}^{n} \times D$.

(T.2) For any fixed $\xi, \widehat{T}_{\lambda}(\xi)$ is an analytic function of $\lambda$ in $D$.

(T. 3) For every $\nu$, there exists a constant $C_{\nu}$ such that

$$
\left|(\partial / \partial \xi)^{\nu} \widehat{T}_{\lambda}(\xi)\right| \leq C_{\nu}(1+|\xi|)^{r-|\nu|},
$$

where $C_{\nu}$ does not depend on $\xi \in \mathbb{G}^{n}$ and on $\lambda \in D$.

Then, the distribution $T_{\lambda}$ is a $C^{\infty}$ function of $x$ in $\mathbb{R}^{n} \backslash\{0\}$ and for any $\beta,(\partial / \partial x)^{\beta} T_{\lambda}$ is a continuous function of $(x, \lambda)$ in $\left(\mathbb{R}^{n} \backslash\{0\}\right) \times D$ and analytic with respect to $\lambda$ in $D$ when each $x \in \mathbb{R}^{n} \backslash\{0\}$ is fixed. Further, as $|x| \rightarrow \infty,(\partial / \partial x)^{\beta} T_{\lambda}$ converges to zero more rapidly than any negative power of $|x|$, where the convergence is uniform roith respect to $\lambda$ in $D$.

Proof. For any positive integer $p$ there exists from (8.16) a constant $C_{p, \beta}$ such that

$$
\left|\Delta_{\xi}^{p}\left\{(i \xi)^{\beta} \widehat{T}_{\lambda}(\xi)\right\}\right| \leq C_{p, \beta}(1+|\xi|)^{r+|\beta|-2 p}, \xi \in \xi^{n},
$$

where $\Delta_{\xi}$ denotes the Laplace operator and $C_{p, \beta}$ is independent of $\lambda \in D$. From $(8 \cdot 17)$

$$
\Delta_{\xi}{ }^{p}\left\{(i \xi)^{\beta} \widehat{T}_{\lambda}(\xi)\right\} \in L^{1}\left(\boldsymbol{\Xi}^{n}\right) \text { if } \quad r+|\beta|-2 p<-n .
$$

Consequently

$$
|x|^{2 p}(\partial / \partial x)^{\beta} T_{\lambda}=\mathscr{F}^{-1}\left[\left(-\Delta_{\xi}\right)^{\rho}\left\{(i \xi)^{\beta} \widehat{T}_{\lambda}(\xi)\right\}\right](x)
$$


is a bounded continuous function of $(x, \lambda)$ and analytic in $\lambda$ where each $x \in \mathbb{R}^{n}$ is fixed. This implies that $T_{\lambda}$ is a $C^{\infty}$ function of $x$ in $\mathbb{R}^{n} \backslash\{0\}$ and $\left|(\partial / \partial x)^{\beta} T_{\lambda}(x)\right| \leq M_{p, \beta}|x|^{-2 p}$ for any $p>0$ and $x \in \mathbb{R}^{n} \backslash\{0\}$, where $M_{p, \beta}$ is a positive constant independent of $\lambda \in D$.

End of proof of Theorem 8.1. It is obvious that $R(\xi, \lambda)$ satisfies (T. 1) and (T. 2) with $D=\Delta=\left\{\lambda ;\left|\operatorname{Re} \lambda-\sigma^{\circ}\right| \leq \delta,|\operatorname{Im} \lambda| \leq \varepsilon^{\circ}\right\}$. Since $Q_{k}(\xi)$ and $P_{k}(\xi)$ are positively homogeneous (matrix valued) functions of degree 1 and degree 0 respectively, which are $C^{\infty}$ in $\boldsymbol{G}^{n} \backslash\{0\}, R(\xi, \lambda)$ satisfies (T.3). By applying Lemma 8.2 to the distribution $F(x ; \lambda)$ we have the statements on $F$ in Theorem 8.1.

In order to describe the asymptotic behavior of the Green's function $G(x ; \lambda)$ of $A-\lambda I$ when the operator $A$ satisfies the conditions $1^{\circ}, 2^{\circ}, 3^{\circ}$, $4^{\circ}$ and $5^{\circ}$, we now recall and introduce some notation. Let $\left\{\lambda_{k}\left(\varsigma^{\complement}\right)\right\}_{|k|=0,1, \ldots, b}$ be the roots reenumerated by $(3 \cdot 33)$ of the equation in $\tau: p(\tau, \hat{\xi})$ $\equiv \operatorname{det}\left(\tau I-\sum_{j=1}^{n} \xi_{j} A_{j}\right)=0$ and let $\lambda_{j k}(\xi)$ be the function defined by $(3 \cdot 29)$. Let $P_{k}(\xi)$ and $P_{j k}(\xi)$ be the matrix valued functions defined by $(3 \cdot 7)$ and $(3 \cdot 30)$ respectively. Put

$$
S_{j k}(\vartheta)=\left\{s ; \lambda_{j k}(s, \vartheta)=1\right\}, \vartheta \in[0,1], 1 \leq j<k \leq b .
$$

$K_{k}(s)$ and $K_{j k}(s, \vartheta)$ denote the Gaussian curvatures of the hypersurfaces $S_{k}$ and $S_{j k}(\vartheta)$ at $s \in S_{k}$ and at $s \in S_{j k}(\vartheta)$ respectively. We denote by $H_{j k}(s, \vartheta)$ the Weingarten operator in the tangent space of $S_{j k}(\vartheta)$ at $s \in S_{j k}(\vartheta)$ and by $\mathfrak{h}_{j k}(\theta, s, \vartheta)$ the orthogonal projection of the vector $\operatorname{grad}\left(\lambda_{j}-\lambda_{k}\right)(s) /\left\langle\operatorname{grad} \lambda_{j k}(s, \vartheta), \theta\right\rangle(\theta \in \Omega)$ on the tangent hyperplane of $S_{j k}(\vartheta)$ at $s \in S_{j k}(\vartheta)$.

Theorem 8. 3. Under the conditions $1^{\circ}, 2^{\circ}, 3^{\circ}, 4^{\circ}$ and $5^{\circ}$ in $\S 1$, the Green's function $G(x ; \lambda)$ of $A-\lambda I$ has the properties (G.1) and (G. 2) in Theorem 8.1 and

(G. 3)* Given a unit vector $\theta \in \Omega$, the asymptotic behavior of $G(x ; \sigma \pm i \varepsilon)$ for $|x| \rightarrow \infty$ along the ray $x=|x| \theta$ are described by the formulas (8.19) with (8.20), and (8.22).

The case where there exist pairs $(j, k) 1 \leq j<k \leq b$ and points $s \in S_{j} \cap S_{k}$ such that for some $\vartheta \in[0,1]$ the normal to $S_{j k}(\vartheta)$ at the 
point $s$ is parallel to the vector: Let $\Lambda$ be the set of all such pair $(j, k)$ and $s_{j k}^{1}(\theta), \cdots, s_{j k}^{r(j, k)}(\theta) \in S_{j} \cap S_{k}$ be all such points and $\vartheta_{j k}^{1}, \cdots$, $\vartheta_{j k}^{r(j, k)}$ the corresponding values of the parameter $\vartheta$. Then

$$
\begin{aligned}
& (\partial / \partial x)^{\nu} G(x ; \sigma \pm i \varepsilon) \\
& =\sum_{(j, k) \in A} \sum_{\iota} C_{ \pm, j, k, \nu}\left(\theta, s_{j k}^{c}(\theta), \vartheta_{j k}^{c}, \operatorname{sgn} \sigma\right)|x|^{-n / 2+1} \\
& \quad \times I_{\nu, s_{j k}^{\imath}(\theta)}(x \operatorname{sgn} \sigma ;|\sigma| \pm i \varepsilon \operatorname{sgn} \sigma)+O\left(|x|^{-n / 2+1-r}\right), \\
& \quad \sigma \pm i \varepsilon \in \Delta_{ \pm}, 0<\gamma<1 / 2,
\end{aligned}
$$

$(8 \cdot 20) \quad C_{ \pm, j, k, \nu}(\theta, s, \vartheta, \operatorname{sgn} \sigma)$

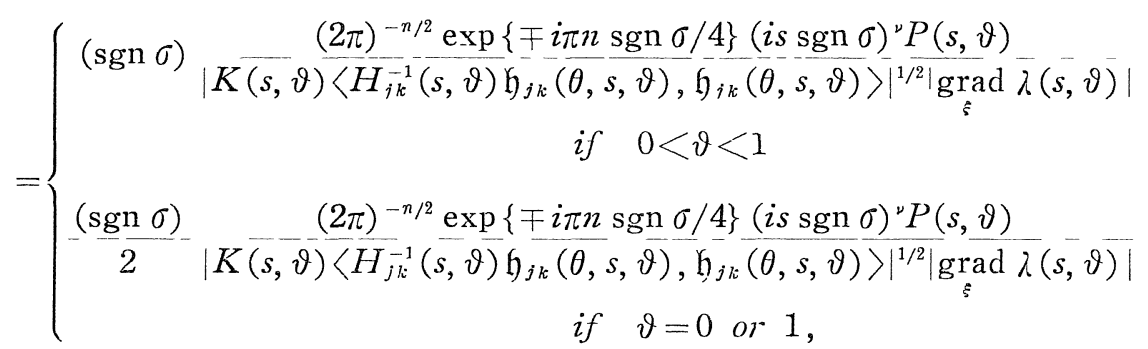

where $I_{\nu, s}(x ; \sigma \pm i \varepsilon)$ is the function defined by $(\tau \cdot 41)$ with $d=1$ and the summation $\sum_{c}$ in (8.19) is taken over c such that $\left\langle\theta, s_{j k}^{l}(\theta)\right\rangle>0$ or that $\left\langle\theta, s_{j k}^{\iota}(0)\right\rangle\langle 0$ according as $G(x ; \sigma+i \varepsilon)$ or $G(x ; \sigma-i \varepsilon)$. Note that the order relation $O$ in $(8 \cdot 19)$ is uniform reith respect to $\sigma \pm i \varepsilon \in \Delta_{ \pm}$but depends on $\gamma$ and that the functions $I_{\nu, s_{j h}^{\prime}(\partial)}(x ;|\sigma| \pm i \varepsilon)$ are bounded continuous functions in $\{x ;|x| \geq 1\} \times \Delta_{ \pm}$.

In particular we have

$$
\begin{aligned}
& (\partial / \partial x)^{\nu} G(x ; \sigma \pm i 0)=\mp 2 \pi \sum_{(j, k) \in \Lambda} \sum_{\zeta} C_{ \pm, j, k, \nu}\left(\theta, s_{j k}^{\ell}(\theta), \vartheta_{j k}^{\iota}, \operatorname{sgn} \sigma\right) \\
& \times\left\langle x, s_{j k}^{\ell}(\theta)\right\rangle|\sigma|^{n / 2+|\nu|}|x|^{-n / 2+1} \exp \left\{i|x|\left\langle\theta, s_{j k}^{\ell}(\theta)\right\rangle\right\} \\
& +O\left(|x|^{-n / 2+1-\gamma}\right),\left|\sigma-\sigma^{\circ}\right| \leq \delta, 0<\gamma<1 / 2 .
\end{aligned}
$$

The case where for any $(j, k)$ with $1 \leq j<k \leq b$ and for $\vartheta \in[0,1]$ there is no point on $S_{j} \cap S_{k}$ at rohich the normal to $S_{j k}(\vartheta)$ is parallel to $\theta:$ Let $s_{k}(\theta)$ be the inverse image of $\theta$ under the Gauss map $S_{k} \ni s \mapsto \boldsymbol{n}(s) \in \Omega . \quad$ Then

$(8 \cdot 22) \quad(\partial / \partial x)^{2} G(x ; \sigma \pm i \varepsilon)$ 


$$
\begin{aligned}
& =(\operatorname{sgn} \sigma)(2 \pi)^{-(n+1) / 2} \sum_{k=1}^{b} \frac{\left(i s_{k}( \pm \theta) \operatorname{sgn} \sigma\right)^{\nu} P_{k}\left(s_{k}( \pm \theta)\right)}{\left|K_{k}\left(s_{k}( \pm \theta)\right)\right|^{1 / 2}\left|\operatorname{grad} \lambda_{k}\left(s_{k}( \pm \theta)\right)\right|} \\
& \quad \times \exp \{\mp i \pi(n-1) \operatorname{sgn} \sigma / 4\} I_{\nu, s_{k}( \pm \theta)}(x \operatorname{sgn} \sigma ;|\sigma| \pm i \varepsilon \\
& \quad \times \operatorname{sgn} \sigma)|x|^{-(n-1) / 2}+O\left(|x|^{-(n-1) / 2-r}\right), \sigma \pm i \varepsilon \in \Delta_{ \pm}, 0<\gamma<1,
\end{aligned}
$$

where

$$
I_{\nu, s}(x ; \sigma \pm i \varepsilon)=\int_{0}^{\infty} \frac{\rho^{(n-1) / 2+|\nu|} \psi(\rho)}{\rho-(\sigma \pm i \varepsilon)} \exp \{i \rho\langle x, s\rangle\} d \rho
$$

The order relation $O$ in (8.22) depends on $\gamma$ and the funclions $I_{\nu, s_{k}(\theta)}(x ;|\sigma| \pm i \varepsilon)$ are bounded continuous in $\{x ;|x|>0\} \times \Delta_{ \pm}$.

In particular.

$$
\begin{aligned}
& (\partial / \partial x)^{\nu} G(x ; \sigma \pm i 0)= \pm i(2 \pi)^{-(n-1) / 2} \\
& \quad \times \frac{\left(i s_{k}( \pm \theta) \operatorname{sgn} \sigma\right)^{\nu} P_{k}\left(s_{k}( \pm \theta)\right)}{\left|K\left(s_{k}( \pm \theta)\right)\right|^{1 / 2}\left|\operatorname{grad} \lambda_{k}\left(s_{k}( \pm \theta)\right)\right|}|\sigma|^{(n-1) / 2+|\nu|} \\
& \quad \times \exp \left\{\mp i \pi(n-1) \operatorname{sgn} \sigma / 4+i \sigma\left\langle x, s_{k}( \pm \theta)\right\rangle\right\}|x|^{-(n-1) / 2} \\
& \quad+O\left(|x|^{-(n-1) / 2-\gamma}\right),\left|\sigma-\sigma^{\circ}\right| \leq \delta, 0<\gamma<1 .
\end{aligned}
$$

The proof of this theorem can be done in the same way as that of Theorem 8.1 using the representation (3.35) instead of (3.21) and applying Theorem 7.4 instead of Theorem 6.3. Note that (8.22) and $(8 \cdot 24)$ are respectively the special case: $\alpha_{1}=\cdots=\alpha_{b}=1$ of $(8 \cdot 3)$ and $(8 \cdot 5)$ combined with $(8 \cdot 2)$ and $(8 \cdot 7)$.

\section{References}

[1] Duff, G. F. D., On wave fronts, and boundary waves, Comm. Pure Appl. Math., 17 (1964), 189-225.

[2] Fedoriuk, M. V., The stationary phase method for multiple integrals, Ž. Vyčisl. Mat. Fiz., 2 (1962), 145-150.

[3] Flanders, H., Differential Forms with Applications to the Physical Sciences, Academic Press, 1963.

[4] Friedrichs, K. O., Über die Spektralzerlegung eines Integraloperators, Math. Ann., 115 (1938), 249-272.

[5] Grušin, V. V., On Sommerfeld-type conditions for a certain class of partial differential equations, Mat. Sb. (N. S.) 61 (1963), 147-174; Amer. Math. Soc. Transl., Ser. 2, 51 (1966), 82-112.

[6] Hicks, N. J., Notes on Differential Geometry, Van Nostrand, 1965.

[7] Hörmander, L., Fourier integral operators. I, Acta Math., 127 (1971), 79-183. 
[8] Kasahara, K. and Yamaguti, M., Strongly hyperbolic systems of linear partial differential equations with constant coefficients, Mem. Coll. Sci., Kyoto Univ., Ser. A, 33(1960), 1-23.

[9] Kobayashi, S. and Nomizu, K., Foundations of Differential Geometry, Vol. 2, Interscience 1969.

[10] Littman, W., Fourier transforms of surface-carried measures and differentiability of surface averages, Bull. Amer. Math. Soc., 69 (1963), 766-770.

[11] - Decay at infinity of solutions of partial differential equations with constant coefficients, Trans. Amer. Math. Soc., 123 (1966), 449-459.

[12] Ludwig, D. and Granoff, B., Propagation of singularities along characteristics with nonuniform multiplicity, J. Math. Anal. Appl., 21 (1968), 556-574.

[13] Matsumura, M., Comportement des solutions de quelques problèmes mixtes pour certains systèmes hyperboliques symétriques à coefficients constants, Publ. RIMS, Kyoto Univ., Ser. A, 4 (1968), 309-359.

[14] - Uniform estimates of elementary solutions of first order systems of partial differential equations, Publ. RIMS, Kyoto Univ., 6 (1970), 293-305.

[15] - On some properties of the fundamental solutions for stationary and nonstationary wave propagation problems. I, La Funkcialaj Ekvacioj, 24 (1972), 433524 (in Japanese).

[16] - Asymptotic behavior at infinity for Green's functions of first order systems with characteristics of nonuniform multiplicity, Reports of Katada Symposium on Partial Differential Equations, (1972), 98-123 (in Japanese).

[17] Matsuura, S., On non-strict hyperbolicity, Conf. on Functional Analysis and Related Topics, Tokyo 1969, Math. Soc. Japan (1970), 171-176.

[18] Mizohata, S., The Theory of Partial Differential Equations, Camb. Univ. Press, 1973.

[19] Schulenberger, J. R. and Wilcox, C. H., A Rellich uniqueness theorem for steadystate wave propagation in inhomogeneous anisotropic media, Arch. Rational Mech. Anal., 41 (1971), 18-45.

[20] Sternberg, S., Lectures on Differential Geometry, Prentice-Hall, 1964.

[21] Strang, G., On strong hyperbolicity, J. Math. Kyoto Univ., 6 (1967), 397-417.

[22] Svensson, S. L., Necessary and sufficient conditions for the hyperbolicity of polynomials with hyperbolic principal part, Ark. Mat., 8 (1970), 145-162.

[23] Vainberg, B. R., Principle of radiation, limit absorption and limit amplitude in the general theory of partial differential equations, Usp. Mat. Nauk, 21 (1966), 111194; Russian Math. Surveys, 21 (1966), 115-193.

[24] Wilcox, C. H., Wave operators and asymptotic solutions of wave propagation problems of classical physics, Arch. Rational Mech. Anal., 22 (1966), 37-78.

[25] - Steady-state wave propagation in homogeneous anisotropic media, Arch. Rational Mech. Anal., 25 (1967), 201-242.

[26] Yamaguti, M. and Kasahara, K., Sur le système hyperbolique à coefficients constants, Proc. Japan Acad., 35 (1959), 547-550. 
